\title{
The Colony Predation Algorithm
}

\author{
Jiaze Tu ${ }^{1}$, Huiling Chen ${ }^{1^{*}}$, Mingjing Wang ${ }^{1}$, Amir H. Gandomi ${ }^{2}$ \\ 1. College of Computer Science and Artificial Intelligence, Wenzhou University, Wenzhou 325035, China \\ 2. Faculty of Engineering and Information Technology, University of Technology Sydney, Ultimo, NSW 2007, Australia
}

\begin{abstract}
This paper proposes a new stochastic optimizer called the Colony Predation Algorithm (CPA) based on the corporate predation of animals in nature. CPA utilizes a mathematical mapping following the strategies used by animal hunting groups, such as dispersing prey, encircling prey, supporting the most likely successful hunter, and seeking another target. Moreover, the proposed CPA introduces new features of a unique mathematical model that uses a success rate to adjust the strategy and simulate hunting animals' selective abandonment behavior. This paper also presents a new way to deal with cross-border situations, whereby the optimal position value of a cross-border situation replaces the cross-border value to improve the algorithm's exploitation ability. The proposed CPA was compared with state-of-the-art metaheuristics on a comprehensive set of benchmark functions for performance verification and on five classical engineering design problems to evaluate the algorithm's efficacy in optimizing engineering problems. The results show that the proposed algorithm exhibits competitive, superior performance in different search landscapes over the other algorithms. Moreover, the source code of the CPA will be publicly available after publication.
\end{abstract}

Keywords: Colony Predation Algorithm, optimization, nature-inspired computing, meta-heuristic, engineering problems Copyright $(\odot)$ The author(s) 2021.

\section{Introduction}

Optimization methods are not limited to single-objective methods, and every single objective idea can be extended for dealing with more classes of problems that have more than one or many objective functions. Common optimization approaches include fuzzy optimization $^{[1]}$, large scale problem solving ${ }^{[2]}$, memetic and hybrid approaches ${ }^{[3]}$, multi-objective optimization (an extension of the single-objective methods) ${ }^{[4]}$, robust optimization, and many objectives ${ }^{[5]}$. Swarm-based stochastic methods involve any type of mathematical form and various inspirations. In recent years, metaheuristic algorithms ${ }^{[4]}$ have attracted much attention and have been extensively used in numerous fields ${ }^{[6-17]}$. Such popularity is attributed to the ability of MAs to solve many possible complex feature spaces in practical problems in neural network-based control ${ }^{[18,19]}$, formation control ${ }^{[20]}$, deep learning models and feature understanding $^{[21,22]}$, adaptive control ${ }^{[23,24]}$, machine learning-based implements ${ }^{[25,26]}$, and artificial intelligence ${ }^{[27]}$. In addition to their characteristic continuity, discreteness, and constraints, MAs can also avoid local optimum, exhibit simplicity, and provide satisfactory solutions to complex problems without the need for gradient information $^{[28,29]}$.

Nowadays, metaheuristic algorithms have gained momentum in new engineering and technical problems ${ }^{[30]}$. In a previous work, we developed numerous MAs motivated by the behavior of biological and physical systems in nature. Metaheuristics can be divided into four categories ${ }^{[31]}$ : Evolutionary Algorithms (EAs), Physics-based algorithms, Human-based algorithms, and Swarm Intelligence (SI) algorithms. Specifically, Holland proposed the Genetic Algorithm (GA), one of the most popular EAs ${ }^{[32]}$, based on Darwin's theory of biological evolution. GA simulates the process of biological evolution, then, searches for the optimal solution in a solution space. The Differential Evolution (DE) algorithm $^{[33]}$ is another popular EA that simulates the cooperative relationship between individuals within a group and the swarm intelligence of competitive production to guide the direction of an optimization search. The stochastic components ${ }^{[34]}$ contribute more variety to the searching patterns in DE. Other established EAs; include Genetic Programming (GP) ${ }^{[35]}$, Evolution

\footnotetext{
*Corresponding author: Huiling Chen
}

E-mail: chenhuiling.jlu@gmail.com 
Strategy $(\mathrm{ES})^{[36]}$, and Evolutionary Programming $(E P)^{[37]}$. Physics-based algorithms are inspired by the physical laws such as Simulated Annealing $(\mathrm{SA})^{[38]}$, which simulates the annealing process of metals in searching for the optimal solution of a problem. The Central Force Optimization (CFO) and Gravitational Search Algorithms (GSA) ${ }^{[39]}$ are some other physics-based metaheuristics. Among the SI algorithms, Grey Wolf Optimizer (GWO) ${ }^{[40]}$, Bat-inspired algorithm $(\mathrm{BA})^{[41]}$, Cuckoo Search $(\mathrm{CS})^{[42]}$, Artificial Bee Colony $(\mathrm{ABC})^{[43]}$, Slime Mould Algorithm (SMA) $)^{[44]}$, Harris Hawks Optimization (HHO ${ }^{[45]}$, Hunger Games Search $(\mathrm{HGS})^{[46]}$, Krill Herd $(\mathrm{KH})^{[47]}$, Moth Search Algorithm $(\mathrm{MSA})^{[48]}$, Monarch Butterfly Optimization (MBO) $)^{[49]}$, Moth Flame Optimization (MFO) ${ }^{[50]}$, Marine Predators Algorithm (MPA) ${ }^{[51]}$, and Whale Optimization Algorithm (WOA) $)^{[52]}$ are widely used. Some human-based optimizers include Tabu Search (TS ${ }^{[53]}$ and Teaching Learning-based Optimization (TLBO $)^{[54,55]}$. While metaheuristic algorithms have their own advantages and disadvantages compared to alternative solvers, they all provide the benefits of simplicity and relatively fast running time.

MAs are divided into four categories that share two principal characteristics: exploration and exploitation. In brief, the randomness of the search is essential to explore the search space as much as possible to ensure that the algorithm has enough exploration capability; thus exploration shows the ability and richness of randomness. Then, the exploitation stage is based on a promising area achieved by the exploration phase, focusing on the local search aptitude. Finding an outstanding balance between these two stages is one of the most challenging metaheuristics problems, which directly relates to the algorithm's performance. Therefore, first-class algorithms, such as $\mathrm{DE}$ and $\mathrm{ABC}$ are the optimal approaches to maintain the right balance between exploration and exploitation.

Even though numerous excellent algorithms have been proposed, the No Free Lunch (NFL) theorem ${ }^{[56]}$ in search indicates shows that none of these methods is a universal best technique for solving any existing or future problems. In other words, each algorithm can only solve one or a class of optimization problems. Therefore, this work developed an effective metaheuristic algo- rithm, the Colony Predation Algorithm (CPA) based on the coexistence of animals. Specifically, CPA mimics the supportive behavior of social animals and predation strategy of hunting animals. In order to improve its superiority, we applied CPA to engineering problems and achieved good results.

The paper is structured as follows. Section 2 presents the background and inspiration for CPA, including its formula, pseudocode, and time complexity. Section 3 discusses the results of the CPA in solving different benchmark problems and the selection of CPA coefficients. Section 4 describes the experiments performed on engineering design problems using the developed algorithm. Section $\mathbf{5}$ summarizes and concludes the paper.

\section{Colony predation algorithm}

This section introduces the inspiration for the CPA and establishes its mathematical model.

\subsection{Inspiration}

To develop our algorithm, we considered the behavior of social animals. Specifically, we focused on colony predation, which is performed by animals to avoid enemies, and increase the probability of hunting success $^{[57]}$. This strategy is commonly portrayed by wolves, hyenas, lions, piranha, and other animals that prey in groups rather than individually ${ }^{[58]}$.

Colony predation can often help predators acquire more prey, thus increasing individual survival rates. Animals living in groups perform colony predation because their survival is closely related to the group, and the behavior of calling partners also makes their search more efficient. For example, hyenas and wolves communicate and cooperate via colony predation to catch more prey than individuals could ${ }^{[59]}$.

Dividing and surrounding prey are the two most commonly used methods that improve the probability of hunting success. Selective abandonment is another strategy that animals adopt when they encounter situations, such as when consumption exceeds their harvest $^{[60]}$. In this behavior, they will abandon their target and choose another which makes their predation more efficient $^{[61]}$.

Herein, we propose a rule for the survival of the 


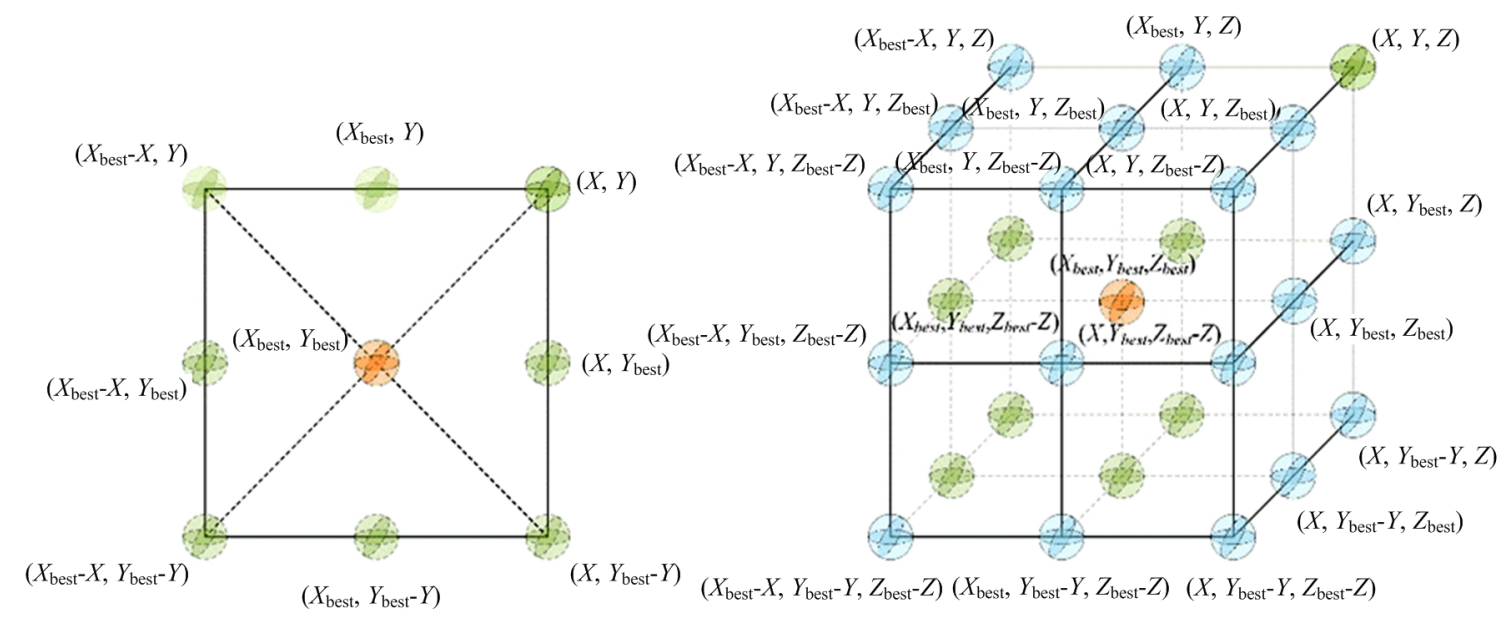

(a)

(b)

Fig. 1 Possible locations in 2 and 3-dimensions.

fittest, where the loser is replaced with the winner. More specifically, the leaders of lion and wolf packs are those who win a fight with the previous leader.

\subsection{Mathematical model}

The mathematical simulation of the position of the algorithm is given here.

Fig. 1 displays the search process of groups and individuals in two and three dimensions, where a predator at position $(X, Y)$ can update its position according to the target's position ( $X_{\text {best }}, Y_{\text {best }}$ ).

Fig. 2 shows how the search agent updates its position based on the predator leader and other predators in the 2D search space. It can be observed that the final position will be a random position within the circle defined by the positions of the predator leader and other predators in the search space. The gray circle represents the final direction of the updated position.

\subsubsection{Communication and collaboration}

Animals who hunt in groups have an increased success rate of predation through communication and cooperation. The following formulas represent individual cooperative communication and food searching behavior:

$$
\stackrel{\mathbf{X}}{\boldsymbol{X}}_{j}^{i}(t+1)=\stackrel{1}{\boldsymbol{X}}_{j}^{i_{1}}(t)+(1-r) \cdot\left(\left(\stackrel{\boldsymbol{X}}{1}_{1}(t)+\stackrel{\mathbf{X}}{2}_{2}(t)\right) / 2\right),
$$

where $r$ is in the range of $[0,1], \dot{X}_{j}^{i_{1}}(t)$ is the individual looking for food; $\stackrel{1}{X}_{1}$ and $\stackrel{1}{X}_{2}$ are the two closest posi- tions to prey in the $j$-th dimension; $j \in 1,2, \ldots, \operatorname{dim}$; and $\dot{X}_{j}^{i}(t+1)$ is the latest updated position of the individual.

\subsubsection{Disperse food}

The first strategy in colony predication drives the prey in different directions, separating the prey from its group. The predation strategy displayed by individuals in search is simulated mathematically as follows:

$$
\stackrel{\hat{X}}{\boldsymbol{X}}(t+1)=\stackrel{1}{\boldsymbol{X}}_{\text {best }}-S \cdot\left(\boldsymbol{r}_{1} \cdot(u b-l b)+l b\right),
$$

where $\stackrel{H}{X}_{(t+1)}$ is the position of a population; $\dot{\boldsymbol{X}}_{\text {best }}$ is the position of food; $S$ represents the strength of prey, where its absolute value decreases from $a$ to 0 with the number of evaluations, $r_{1}$ represents $\left[R_{1} ; R_{2} ; R_{3} ; \ldots R_{j}\right]$; and $\left[R_{1} ; R_{2} ; R_{3} ; \ldots R_{j}\right]$ is the random number of $[0,1] ; j=\operatorname{dim}$ is the dimension of the population; and, $u b$, and $l b$ are the upper and lower bounds, respectively.

The formula of $S$ is as follows:

$$
\begin{gathered}
S_{0}=a-t \cdot\left(\frac{a}{N}\right), \\
S=2 \cdot S_{0} \cdot r_{2}-S_{0},
\end{gathered}
$$

where $N$ represents the number of individuals; $S_{0}$ decreases from $a$ to 0 with the number of evaluations; $t$ represents the current number of evaluations; and, $r_{2}$ is a random number of $[0,1]$. 


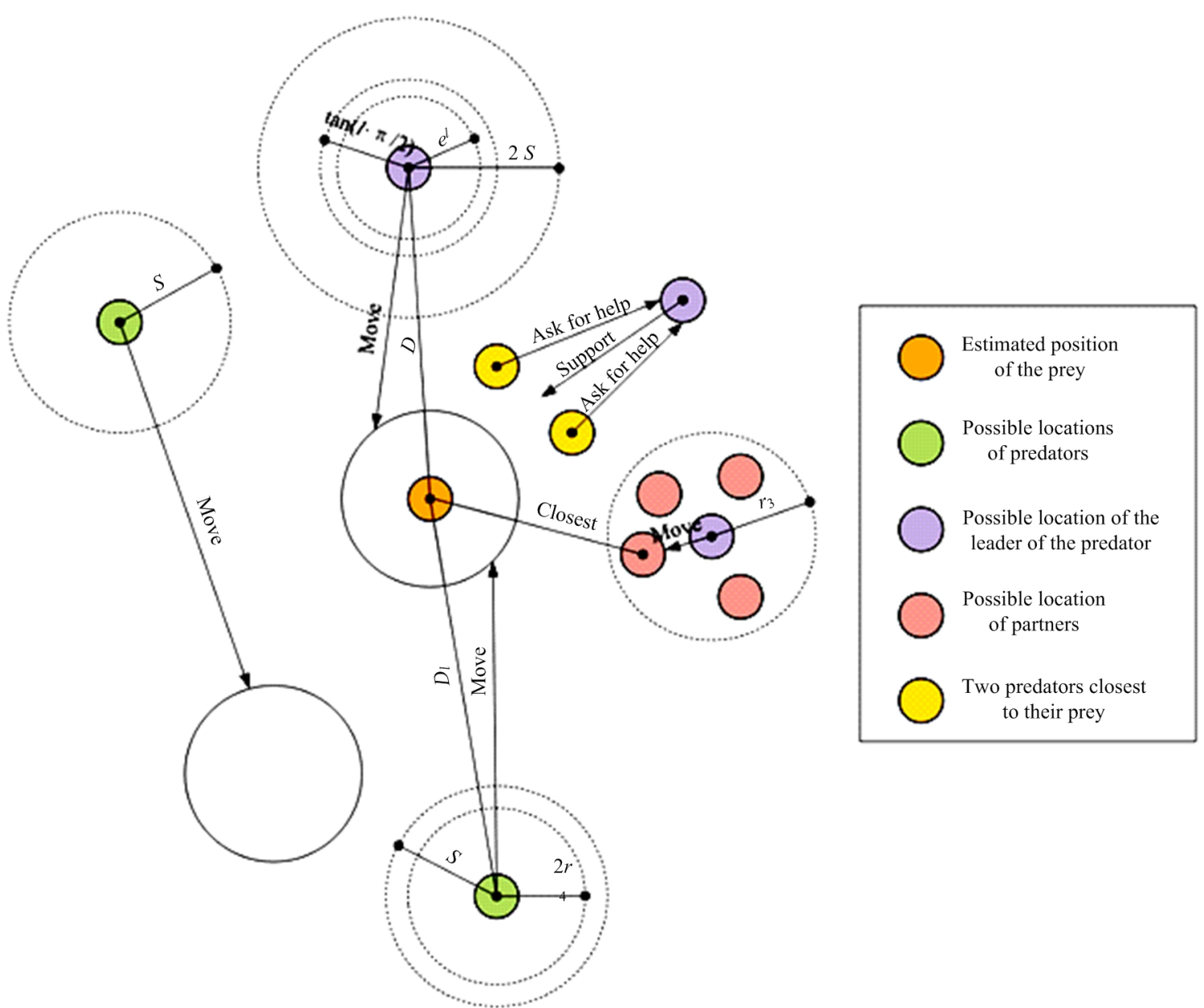

Fig. 2 Position updating in CPA.

The formula of $a$ is as follows:

$$
a=e^{w-2 w \cdot\left(1-\frac{t}{M a x F E s}\right)},
$$

where $w=9$.

\subsubsection{Encircle food}

The hunting group will use the second strategy to surround and approach the prey. This stage can be represented by mathematical simulation as:

$$
\stackrel{\mathrm{r}}{\boldsymbol{X}}(t+1)=\stackrel{\mathrm{r}}{\boldsymbol{r}}_{\text {best }}-2 S \cdot \boldsymbol{D} \cdot e^{l} \cdot \tan \left(\frac{\pi}{4} l\right),
$$

where $\boldsymbol{D}$ is the distance between the current individual and prey, and $\boldsymbol{D}$ is different between different individuals; $l$ is a random number of $[0,1]$; and, $\tan \left(\frac{\pi}{4} l\right)$ is the encirclement curve of the hunter.

The formula of $\boldsymbol{D}$ is as follows:

$$
\boldsymbol{D}=\left|\stackrel{\mathrm{r}}{\boldsymbol{X}}_{\text {best }}-\stackrel{\mathrm{r}}{\boldsymbol{X}}(t)\right|,
$$

where $\stackrel{1}{\boldsymbol{X}}(t)$ represents the current hunter population.

The execution probabilities of these two predatory strategies are expressed as:

$$
\stackrel{\mathrm{X}}{\boldsymbol{r}}(t+1)= \begin{cases}\stackrel{\mathrm{I}}{\boldsymbol{X}}_{\text {best }}-S \cdot\left(\boldsymbol{r}_{1} \cdot(u b-l b)+l b\right) & r_{7} \geq 0.5 \\ \stackrel{\mathrm{r}}{\boldsymbol{X}_{\text {best }}-2 S \cdot \boldsymbol{D} \cdot e^{l} \cdot \tan \left(\frac{\pi}{4} l\right)} & r_{7}<0.5\end{cases}
$$

\subsubsection{Supporting closest individual}

Considering that the group may encounter difficulties in hunting prey, the nearest individual calls for peer support, which can be expressed as follows:

$$
\stackrel{1}{\boldsymbol{X}}(t+1)=\stackrel{\mathfrak{P}}{\boldsymbol{P}}_{\text {nearest }},
$$

where $\stackrel{\stackrel{P}{\boldsymbol{P}}}{\text { nearest }}_{\text {is }}$ the location of the nearest predator in 
the support group; and $\stackrel{\boldsymbol{P}}{\boldsymbol{P}}$ is the predator closest to the prey.

The formula of $\stackrel{\boldsymbol{P}}{\boldsymbol{P}}$ is as follows:

$$
\stackrel{\mathbf{P}}{\boldsymbol{P}}=r_{3} \cdot \stackrel{\boldsymbol{X}}{\boldsymbol{X}}_{j}^{i}
$$

where $j \in 1,2, \ldots, \operatorname{dim} ; r_{3}$ represents $\left[R_{1} ; R_{2} ; R_{3} ; \ldots R_{j}\right]$ and $\left[R_{1} ; R_{2} ; R_{3} ; \ldots R_{j}\right]$ is the random number of $[0,1]$.

\subsubsection{Searching for the food}

The remaining individuals will find an alternative food source if no prey is found nearby or food too far away from the prey. This behavior can be expressed as follows:

$$
\begin{aligned}
& \boldsymbol{D}_{1}=\left|2 r_{4} \cdot \stackrel{\mathrm{r}}{\boldsymbol{X}}_{\text {rand }}-\stackrel{\mathrm{r}}{\boldsymbol{X}}(t)\right| \text {, } \\
& \stackrel{\mathbf{X}}{\boldsymbol{X}}(t+1)=\stackrel{\mathfrak{X}}{\boldsymbol{X}}_{\text {rand }}-S \cdot \boldsymbol{D}_{1},
\end{aligned}
$$

where $\boldsymbol{D}_{1}$ denotes the distance of random group movement, $r_{4}$ is a random number of $[0,1]$, and $\stackrel{\perp}{\boldsymbol{X}}_{\text {rand }}$ is a new individual position formed randomly by individuals.

The formula of $\stackrel{\mathfrak{X}}{\boldsymbol{X}}_{\text {rand }}$ is as follows:

$$
\stackrel{\stackrel{X}{X}}{\text { rand }}=r_{5} \cdot((u b-l b)+l b),
$$

where $u b$ and $l b$ are the upper and lower bounds of functions, respectively, $\operatorname{dim}$ is the dimension of population; $r_{5}$ represents $\left[R_{1} ; R_{2} ; R_{3} ; \ldots R_{j}\right]$; and $\left[R_{1} ; R_{2} ; R_{3} ; \ldots R_{j}\right]$ is the random number of $[0,1]$.

The probability of implementing supporting the closest individual and searching for the food is determined by the $r_{6}$ between the group and prey, which can be expressed as follows:

$$
\stackrel{\mathrm{r}}{\boldsymbol{X}}(t+1)=\left\{\begin{array}{ll}
\stackrel{\mathrm{r}}{\boldsymbol{P}}_{\text {nearest }} & \left|r_{6}\right| \leq 1 \\
\stackrel{\mathrm{X}}{\boldsymbol{X}}_{\text {rand }}-S \cdot \boldsymbol{D}_{1} & \left|r_{6}\right|>1
\end{array},\right.
$$

where $r_{6}$ is a random number of $[-2,2]$.

Eq. (10) and Eq. (12) describe the randomness so the searched solution can traverse the solution space. Specifically, Eq. (10) utilizes the current solution as a reference and randomly generates multiple random solutions, so that the current solution is replaced by the generated optimal solution to achieve the purpose of avoiding the local optimum. The current solution is then updated by a completely random solution and replaced by the current solution in Eq. (12), which is equivalent to searching randomly for fresh prey when the current prey cannot be captured.

When the predator's position exceeds the upper or lower limit, we introduce the rule of survival of the fittest in nature and replace the position beyond the limit with the current optimal position. The specific formula is as follows:

$$
\stackrel{\mathfrak{I}}{\boldsymbol{X}}_{\mathrm{ofb}}(j)=\stackrel{\mathfrak{L}}{\boldsymbol{X}}_{\text {best }}(j),
$$

where $\stackrel{\mathbf{X}}{\boldsymbol{X}}_{\mathrm{ofb}}(j)$ is the position beyond the boundary and $\stackrel{\boldsymbol{X}}{\text { best }}_{\text {(j) }}(j)$ is the optimal position, $j \in 1,2, \ldots, \mathrm{dim}$.

The CPA proposed in this paper imitates the process of colony predation. We simplify the algorithm as much as possible to maximize its scalability. Algorithm 1 shows the pseudo-code of the CPA.

Fig. 3 displays the flowchart of CPA, where MaxFEs represents the maximum number of evaluations, value indicates the fitness of each evaluation, $N$ denotes the population number, and $\operatorname{dim}$ is the problem's dimension.

As a unique optimizer with stable performance, CPA has exhibited high potential to solve optimization cases, which is attributed to the following reasons:

(1) The idea of selective abandonment adopted in the algorithm breaks the boundary between exploration and exploitation and increases the exploratory tendency even in the middle and later stages of exploitation, which further helps to prevent from dropping into the Local Optimum (LO).

(2) The value of the current optimal solution is used rather than the value of transboundary individuals, which avoids the problem of excessive spatial dispersion.

(3) The introduction of adaptive weight $a$ ensures that the algorithm can quickly transit from exploration to exploitation in the early stage, allowing more time to execute the exploitation, while $S$ guarantees the perturbation of the algorithm so it will not fall into the LO prematurely.

(4) Innovative use of the communication and cooperation mechanism can increase the diversity in the early stage and strengthen exploration of the local solution space in the later stage. 


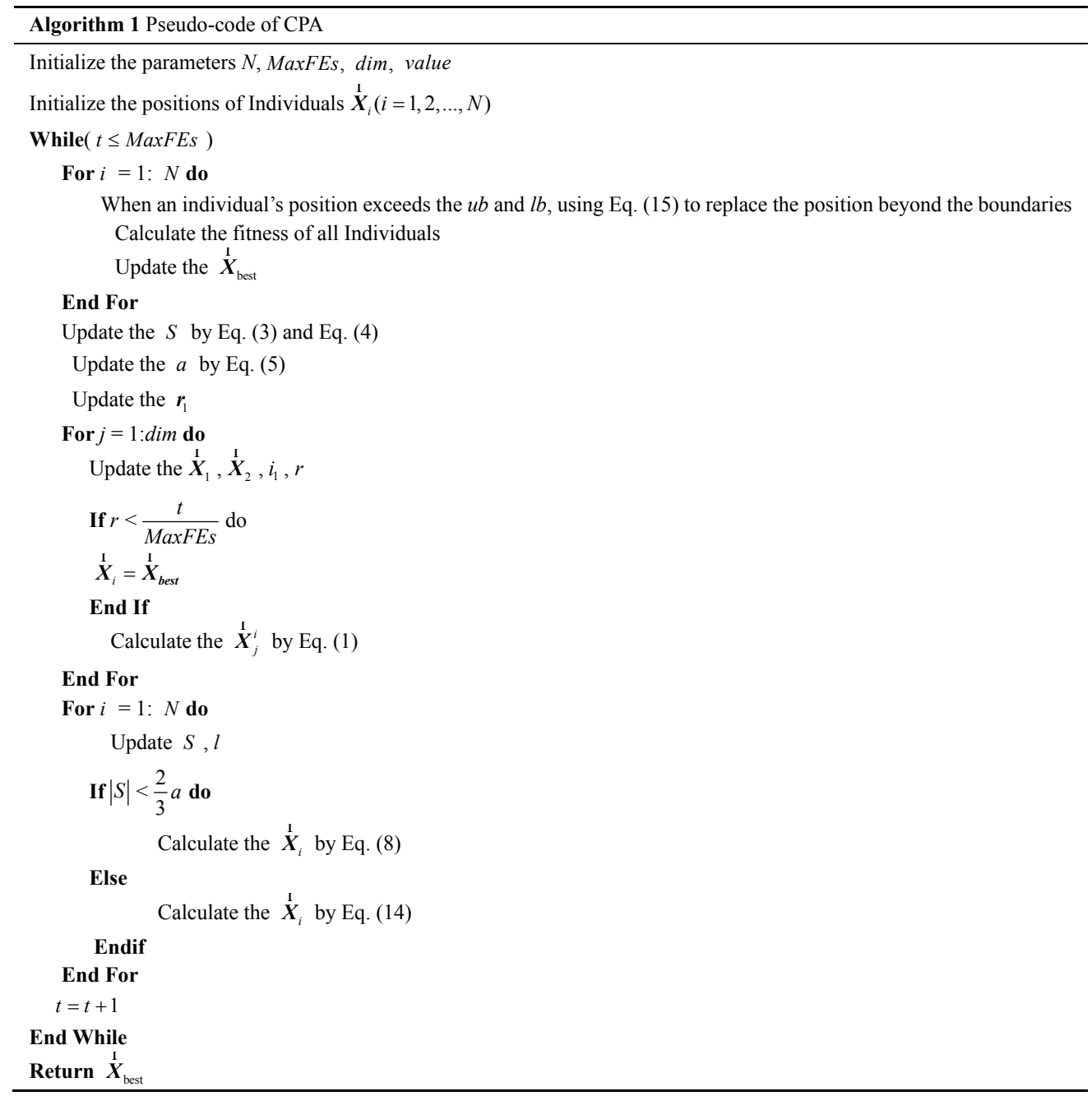

Fig. 4 further demonstrates the authenticity of the preceding ideas. First, we chose test function, F1, to prove point 2 and $\mathrm{F} 12$ to prove the influence of points 1, 3 , and 4 on CPA's exploration ability. In the figure, CPA is in red, CPA with linear parameter $a$ is in blue, CPA with the communication and cooperation mechanism removed is in green, and CPA with the abandonment mechanism removed is pink. Comparing (d), (c), and (d) proves that the current optimal value can effectively reduce the distribution of space midpoint and improve the performance. It can be seen from (f), (g), and (h), it can be seen that the CPA variants fall into the LO earlier than CPA on its own, thus proving that points 1,3 , and 4 are correct.

\subsubsection{Computational complexity analysis}

In the proposed CPA initialization, fitness evalua- tion, communication and collaboration, parameter updating, and location updating are performed. In the respective functions, $N$ is the number of individuals in the population, $D$ is the dimension of the problem, and MaxFEs indicates the maximum number of evaluations. $O(N)$ refers to the computational complexity of initialization, fitness evaluation, parameter updating, and communication and collaboration, while the computational complexity of location updating is $O\left(N \times\left(1+\frac{D+N^{2}}{4}\right)\right)$. From this, we can obtain the com$O\left(N \times\left(1+\right.\right.$ MaxFEs $\left.\left.\times N \times\left(1+\frac{D+N^{2}}{4}\right)\right)\right)$.

\section{Experiments and results}

This section compares the proposed CPA with a number of conventional and recent optimizers in the 


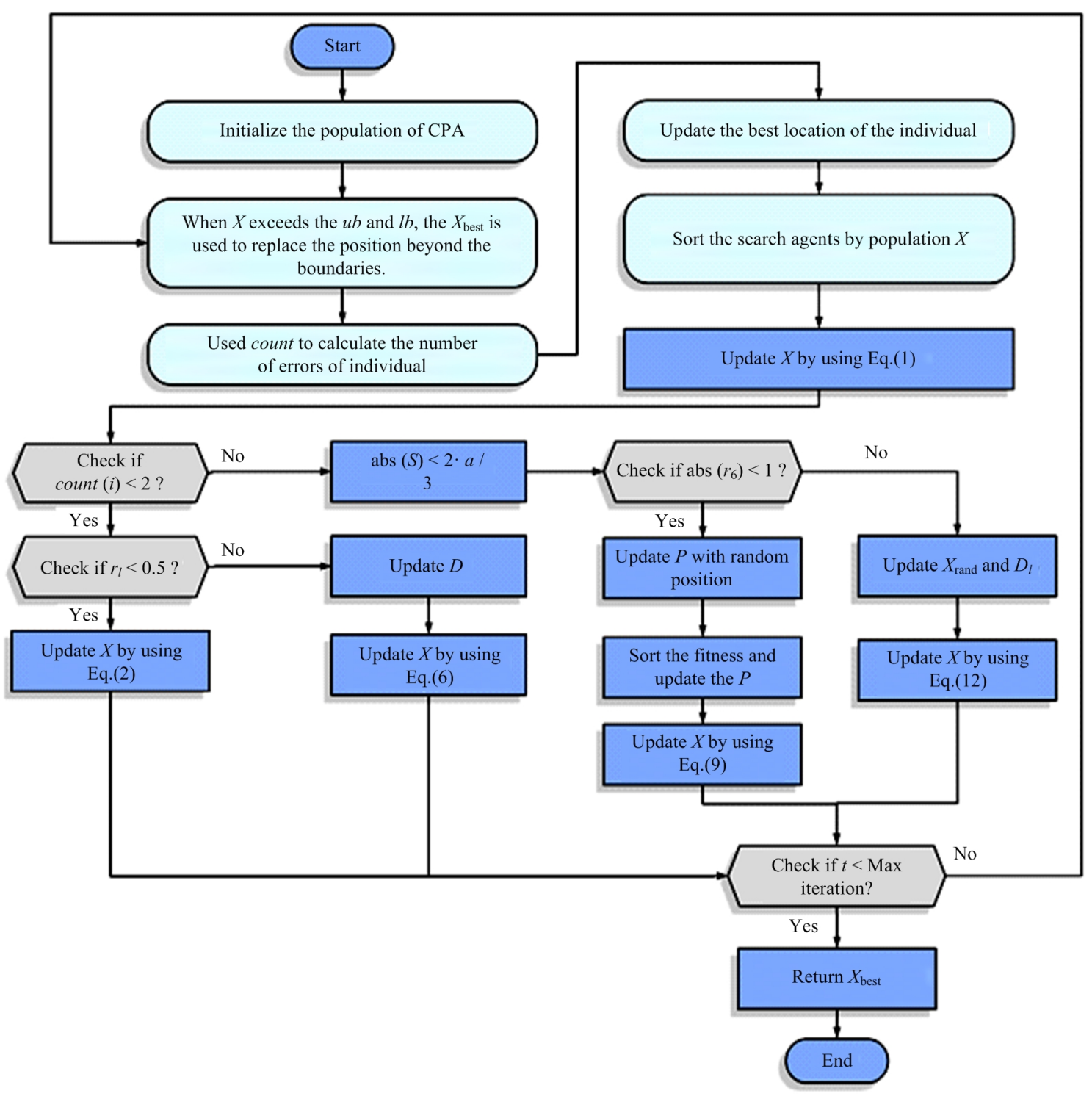

Fig. 3 Flowchart of CPA.

field. All experiments were conducted on Windows Server 2008 R2 operating system with Intel (R) Xeon (R) CPU E5-2650 v4 $(2.20 \mathrm{GHz})$ and $128 \mathrm{~GB}$ of RAM. We coded all algorithms for comparison using MATLAB R2014b.

\subsection{Benchmark function validation}

The algorithm was tested on 53 functions, among which F1 F23 are benchmark functions (Table A1) and F24 F53 are CEC2014 functions (Table A2). These functions cover both the monomodality and multimodality of problems. In the respective functions, Dim represents the dimension of the function, Range refers to the definition domain of the function, and $f_{\min }$ reveals the optimal solution of the function.

All experiments were conducted under the same conditions to ensure the fairness of the experimental

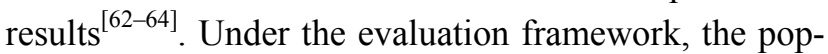
ulation size was set to 30 , and the number of evaluations and dimensions were set to 1000 and 30, respectively. This ensures that there is no partiality of the system, as per literature ${ }^{[26,65-72]}$. At the same time, we tested each algorithm 30 times to exclude the influence of random factors. We applied the Friedman test and the Wilcoxon-signed rank test to compare the performances of the algorithms. The Friedman test is a non-parametric 
F1

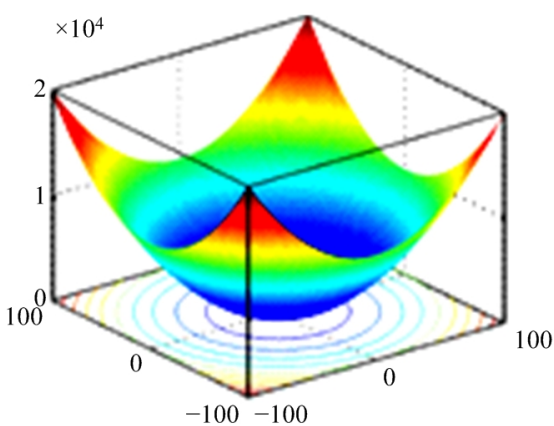

(a)

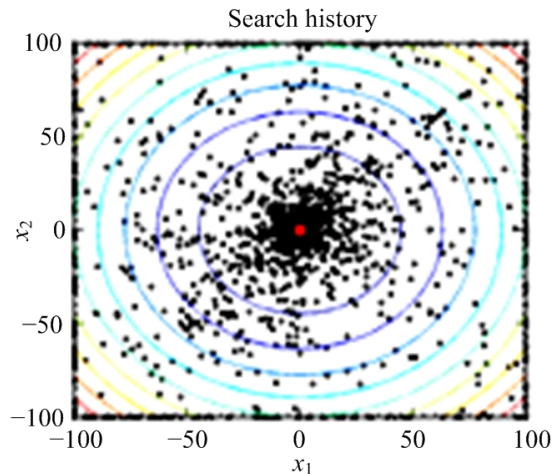

(c)

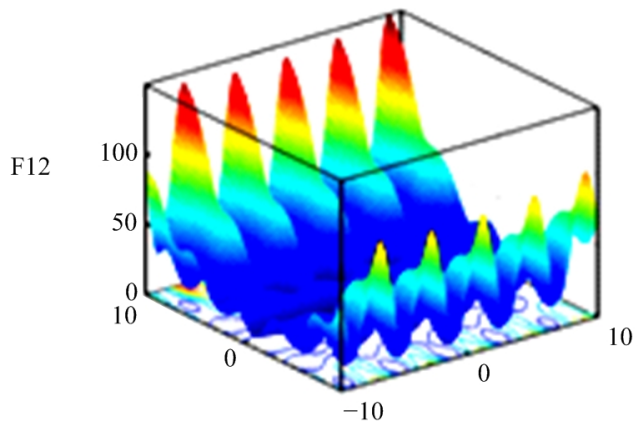

(e)

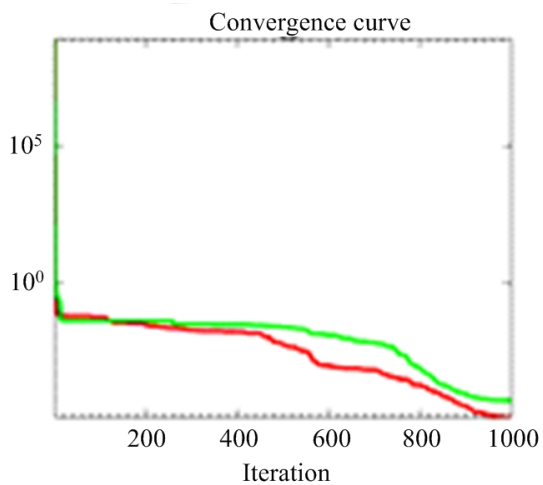

(g)

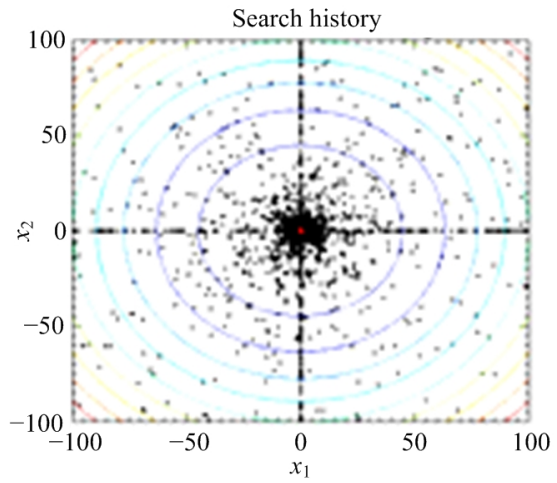

(b)

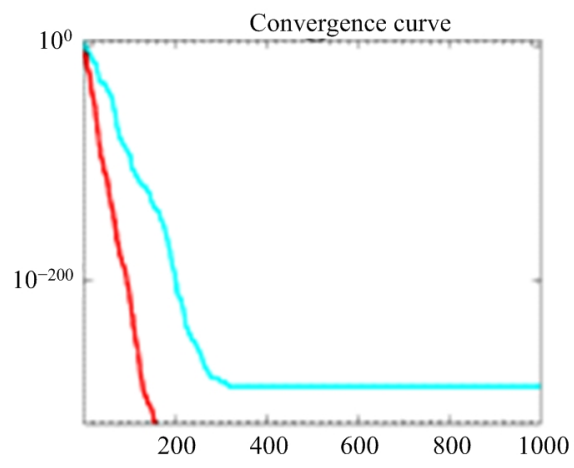

(d)

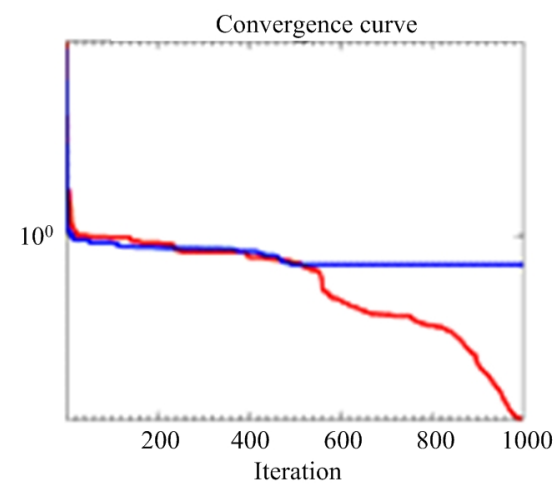

(f)

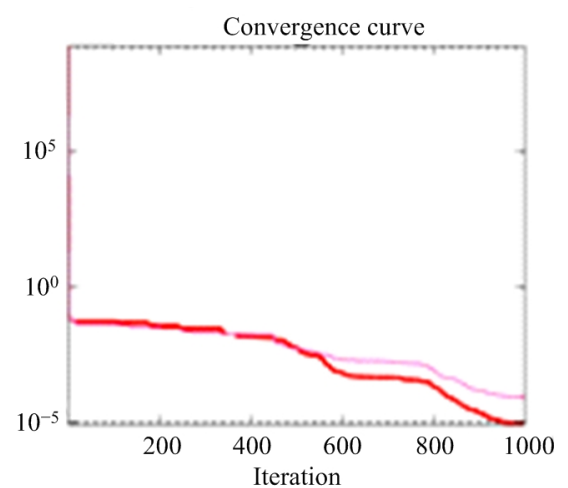

(h)

Fig. 4 Comparison of the mechanisms of each part of CPA: (a) Three-dimensional image of function; (b) lattice diagram of CPA; (c) CPA that uses the boundary value to process out of boundary; (d) comparison diagram of the convergence curve, in which red is the CPA. 
F4

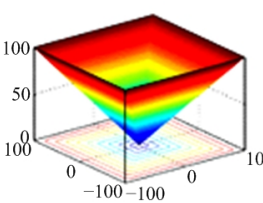

F5
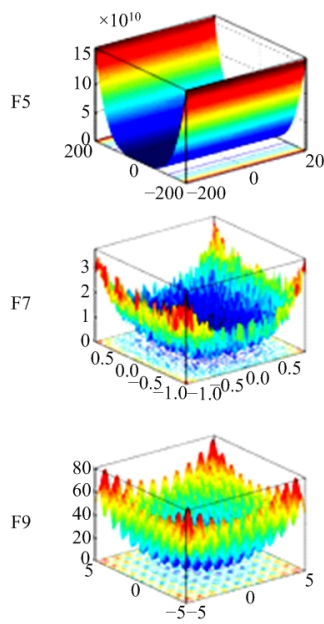
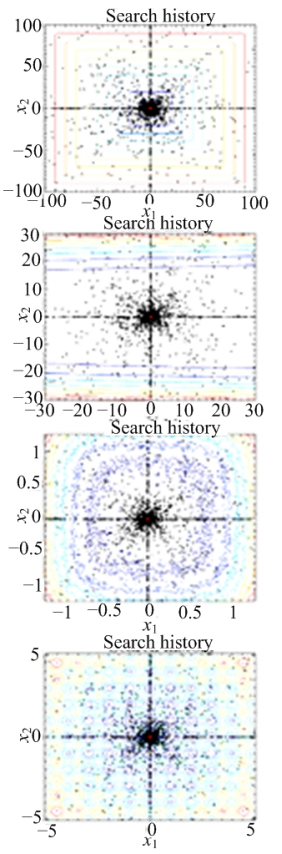
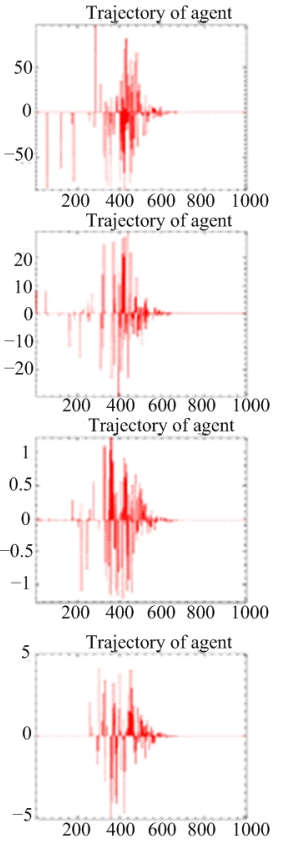
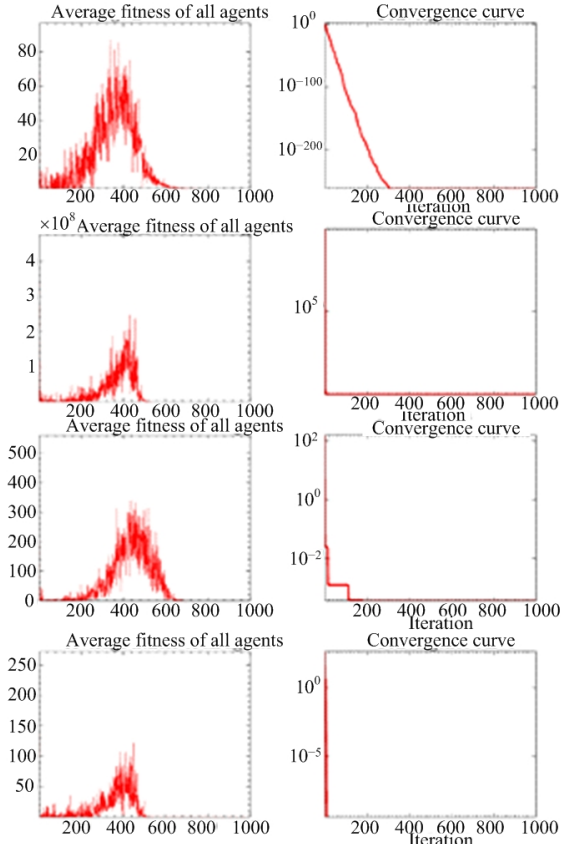

Fig. 5 Qualitative analysis of CPA.

statistical program that allows us to do further analysis of the algorithm's average performance ranking. The Wilcoxon test is often used for statistical testing, in which a $p$-value less than 0.05 indicates that the performance of CPA performance is better than its competitors.

\subsection{Qualitative analysis}

Fig. 5 presents the results of a qualitative analysis of CPA on benchmark functions. F4, F5, F7 and F9 (from Table A1) to analyze its exploration and exploitation ability. We selected 2 single-mode functions and 2 multi-mode functions as the evaluation criteria. The results consist of four major components: 1) The search history shows the location and distribution of individuals in each evaluation; 2) the trajectory of the first individual reveals the individual's motion law throughout the evaluation process; 3 ) the average fitness of all individuals monitors how the average fitness of the entire population changes during the optimization process; and 4) the convergence behavior reveals the changing trend of optimal fitness.

We can see that an individual explored most of the solution space according to its historical position, revealing that the algorithm has strong search ability and can avoid falling into local optimal solutions on complex multimode functions. At the same time, we can also observe that most of the search locations are around the optimal solution, which suggests that the algorithm can be accurately developed in the target area. Moreover, the convergence speed is quick on all functions except F4, yet it can still reach the medium term's optimal value.

The trajectory diagram shows that individuals have sharp fluctuations in the initial stage and the medium term of search. The fluctuation coverage exceeds $50 \%$ of the solution space, proving that the CPA has a powerful search ability. The algorithm can find the optimal solution quickly from the function image and, thus, performs very well on simple single-mode or complex multimode functions.

The algorithm tends to converge quickly in the early stages of evaluation by monitoring the overall average fitness. CPA obtained the best average fitness of all functions, except F15 and F23, in a concise time with repetition. Although the average fitness fluctuated at times, its gradual decrease reflects the algorithm's super search and pioneering ability. The convergence curve further reveals the algorithm's fast convergence speed.

\subsubsection{Comparison with other algorithms}

CPA was further compared with 11 traditional MAs, including $\mathrm{SCA}^{[73]}, \mathrm{SSA}^{[74]}, \mathrm{GWO}^{[40]}, \mathrm{MFO}^{[50]}, \mathrm{WOA}^{[52]}$, 
$\mathrm{ABC}, \mathrm{GSA}, \mathrm{PSO}^{[75]}, \mathrm{DE}, \mathrm{FA}^{[76]}$, and BA. Table A3 lists the parameters of these algorithms, which were taken from the classical algorithm without any modification.

The data in Table 1 provides the results of CPA compared with other traditional MAs based on average results and ranking of the Friedman test in the last column. In the table, "+" indicates that the CPA's performance is better than that of the corresponding algorithm; "-" means that CPA performance is worse than the corresponding algorithm; and "=" CPA and the corresponding algorithm. The average (AVG) and standard deviation (STD) values were obtained from the Friedman test and correspond to the algorithm's average ranking result. We can intuitively find from Table 1showsthat CPA ranks first and that it was challenging for the competitive algorithms to defeat CPA on most of the 53 functions. In addition, the Friedman rank of CPA is only 3.20 , which is much smaller than the other algorithms. Compared with the second DE, CPA's average is about 0.9 lower than DE. CPA also ranks in the top three on F6, F13, F21, F22, F23, F25, F26, F27, F29, F32, F34, and F38. Moreover, because the STD of CPA reached 0 on F1, F2, F3, F4, F9, F17, F47, and F48, we can conclude that the CPA has excellent performance.

Table A4 shows the results of the Wilcoxon test most of the $p$-values are less than 0.05 , accounting for $88 \%$ of all data. Even in SCA, only two p-values are higher than 0.05. CPA is far superior to the $\mathrm{DE}$ and $\mathrm{ABC}$ on most functions, although the number of $p$-values higher than 0.05 accounts for $13 \%$ and $19 \%$ of the total, respectively. This further shows that the CPA has a strong statistical significance.

Fig. 6 reveals that the convergence speed of the CPA is very fast, which provides an index to judge the algorithm's performance. CPA found the optimal value in the early stage of $F 1$ and F4, so there is no CPA curve in the image. For F1, F4, F7, F15, F46, F50, and F53, CPA also converged the fastest among all algorithms, while some other algorithms converged quite slow, and fell into local optima. Functions F46, F50, and F53 demonstrate that CPA has high accuracy in solving problems and can quickly find the global optimum at the beginning of the evaluation. While the convergence speed of some other algorithms was also very fast, their accuracy in finding the solution was not as high as CPA's, and the solution found by CPA is better. Even though its CPA convergence speed was slower on F24, CPA still found the global optimum before other algorithms; some of which even fell into the local optimum at the beginning of the evaluation. It can be concluded from the performance on F15 and F35 functions that CPA has a strong ability for global exploration, can find the target area in the early stage and fully balances the exploration and exploitation, further confirming its superior performances.

\subsubsection{Comparison with advanced MAs}

To further illustrate its superior CPA was also compared with 9 well-known improved algorithms: Modified Sine Cosine Algorithm (m_SCA) ${ }^{[77]}$, Opposition-Based Sine Cosine Algorithm (OBSCA) ${ }^{[78]}$, Modified Whale Optimization Algorithm (MWOA) ${ }^{[79]}$, Improved Whale Optimization Algorithm (IWOA) ${ }^{[80]}$, Bat Algorithm based on Collaborative and Dynamic Learning of Opposite Population (CDLOBA $)^{[81]}$, Chaotic Bat Algorithm $(\mathrm{CBA})^{[82]}$, Chaotic and Gaussian Particle Swarm Optimization (CGPSO) ${ }^{[83]}$, Particle Swarm Optimization with an Aging Leader and Challengers (ALCPSO $^{[84]}$, and Differential Evolution Algorithm based on Chaotic Local Search (DECLS) ${ }^{[85]}$.

The data in Table 2 reveal that the CPA has an excellent performance in both single-mode and multimode functions, especially in the fixed dimension multimode function. The average of CPA results is 2.88 , which is much smaller than the average of other algorithms. For instance, this average is one-fifth the average of MWOA, m_SCA, OBSCA, IWOA, CDLOBA, CBA, and CGPSO algorithms, the strongest algorithms defeated CPA on only 11 out of the 53 functions, and MWOA did not beat CPA on any function. This shows that the CPA has a strong optimization ability.

Table A5 lists the $p$-values of CPA and the advanced algorithm on all test functions and shows that all values of MWOA are less than 0.05 . We can also see that the difference between the values is more than 0.05 , although the number of ALCPSO's values greater than 0.05 approaches 14 . These test results further confirm that the CPA is superior to other competitive algorithms.

F1, F4, F10, and F20 belong to 23 benchmark functions, while F26, F28, F35, F39, F43, F50, F52, and 
Table 1 Comparison results of CPA with traditional algorithms on the 53 functions

\begin{tabular}{|c|c|c|c|c|c|c|}
\hline \multirow{2}{*}{ Algorithm } & \multicolumn{2}{|c|}{$\mathrm{F} 1$} & \multicolumn{2}{|c|}{$\mathrm{F} 2$} & \multicolumn{2}{|c|}{ F3 } \\
\hline & AVG & STD & AVG & STD & AVG & STD \\
\hline CPA & $0.000 E+00$ & $0.000 E+00$ & $0.000 E+00$ & $0.000 E+00$ & $0.000 E+00$ & $0.000 \mathrm{E}+00$ \\
\hline PSO & $9.648 \mathrm{E}+01$ & $1.103 \mathrm{E}+01$ & $4.569 \mathrm{E}+01$ & $3.989 \mathrm{E}+00$ & $1.703 \mathrm{E}+02$ & $2.269 \mathrm{E}+01$ \\
\hline $\mathrm{DE}$ & $1.07 \mathrm{E}-241$ & $0.000 \mathrm{E}+00$ & $1.95 \mathrm{E}-142$ & $2.21 \mathrm{E}-142$ & $2.655 \mathrm{E}+02$ & $1.352 \mathrm{E}+02$ \\
\hline $\mathrm{ABC}$ & $4.881 \mathrm{E}-16$ & $4.751 \mathrm{E}-17$ & $1.209 \mathrm{E}-15$ & $1.135 \mathrm{E}-16$ & $8.885 \mathrm{E}+02$ & $3.661 \mathrm{E}+02$ \\
\hline SCA & $4.993 \mathrm{E}-85$ & $2.732 \mathrm{E}-84$ & $2.216 \mathrm{E}-86$ & $1.182 \mathrm{E}-85$ & $8.397 \mathrm{E}-02$ & $3.090 \mathrm{E}-01$ \\
\hline SSA & $3.040 \mathrm{E}-09$ & $7.088 \mathrm{E}-10$ & $1.025 \mathrm{E}-01$ & $1.784 \mathrm{E}-01$ & $3.447 \mathrm{E}-08$ & $1.075 \mathrm{E}-08$ \\
\hline BA & $6.230 \mathrm{E}-01$ & $3.947 \mathrm{E}-01$ & $2.626 \mathrm{E}+00$ & $9.802 \mathrm{E}-01$ & $2.328 \mathrm{E}-01$ & $2.311 \mathrm{E}-01$ \\
\hline FA & $1.117 \mathrm{E}+04$ & $1.084 \mathrm{E}+03$ & $4.761 \mathrm{E}+01$ & $3.679 \mathrm{E}+00$ & $1.839 \mathrm{E}+04$ & $2.322 \mathrm{E}+03$ \\
\hline MFO & $2.667 \mathrm{E}+03$ & $5.208 \mathrm{E}+03$ & $3.400 \mathrm{E}+01$ & $2.207 \mathrm{E}+01$ & $1.529 \mathrm{E}+04$ & $1.135 \mathrm{E}+04$ \\
\hline WOA & $0.000 E+00$ & $0.000 E+00$ & $0.000 E+00$ & $0.000 E+00$ & $3.185 \mathrm{E}+00$ & $5.625 \mathrm{E}+00$ \\
\hline GWO & $0.000 E+00$ & $0.000 E+00$ & $0.000 E+00$ & $0.000 E+00$ & $5.74 \mathrm{E}-272$ & $0.000 \mathrm{E}+00$ \\
\hline GSA & $1.441 \mathrm{E}+01$ & $1.639 \mathrm{E}+00$ & $1.520 \mathrm{E}+01$ & $8.396 \mathrm{E}-01$ & $2.801 \mathrm{E}+02$ & $5.079 \mathrm{E}+01$ \\
\hline \multirow{2}{*}{ Algorithm } & \multicolumn{2}{|c|}{$\mathrm{F} 4$} & \multicolumn{2}{|c|}{ F5 } & \multicolumn{2}{|c|}{ F6 } \\
\hline & AVG & STD & AVG & STD & AVG & STD \\
\hline CPA & $0.000 E+00$ & $0.000 E+00$ & $2.143 E+01$ & $1.578 \mathrm{E}-01$ & $2.115 \mathrm{E}-26$ & $2.834 \mathrm{E}-26$ \\
\hline PSO & $3.638 \mathrm{E}+00$ & $2.122 \mathrm{E}-01$ & $7.769 E+04$ & $1.557 \mathrm{E}+04$ & $9.153 \mathrm{E}+01$ & $9.514 \mathrm{E}+00$ \\
\hline $\mathrm{DE}$ & $1.535 \mathrm{E}-21$ & $7.511 \mathrm{E}-21$ & $3.085 \mathrm{E}+01$ & $1.921 \mathrm{E}+01$ & $0.000 E+00$ & $0.000 \mathrm{E}+00$ \\
\hline $\mathrm{ABC}$ & $1.434 \mathrm{E}+00$ & $4.321 \mathrm{E}-01$ & $2.382 \mathrm{E}-01$ & $5.617 \mathrm{E}-01$ & $4.795 \mathrm{E}-16$ & $7.891 \mathrm{E}-17$ \\
\hline SCA & $2.310 \mathrm{E}-04$ & $1.198 \mathrm{E}-03$ & $2.731 \mathrm{E}+01$ & $6.822 \mathrm{E}-01$ & $3.485 \mathrm{E}+00$ & $2.589 \mathrm{E}-01$ \\
\hline SSA & $1.578 \mathrm{E}-01$ & $3.946 \mathrm{E}-01$ & $3.753 \mathrm{E}+01$ & $3.316 \mathrm{E}+01$ & $3.006 \mathrm{E}-09$ & $7.252 \mathrm{E}-10$ \\
\hline BA & $4.530 \mathrm{E}+00$ & $6.079 \mathrm{E}+00$ & $2.840 \mathrm{E}+02$ & $4.338 \mathrm{E}+02$ & $6.433 \mathrm{E}-01$ & $3.621 \mathrm{E}-01$ \\
\hline FA & $3.930 \mathrm{E}+01$ & $2.088 \mathrm{E}+00$ & $7.156 \mathrm{E}+06$ & $1.402 \mathrm{E}+06$ & $1.090 \mathrm{E}+04$ & $1.164 \mathrm{E}+03$ \\
\hline MFO & $6.410 \mathrm{E}+01$ & $9.421 \mathrm{E}+00$ & $2.689 \mathrm{E}+06$ & $1.459 \mathrm{E}+07$ & $1.010 \mathrm{E}+03$ & $3.082 \mathrm{E}+03$ \\
\hline WOA & $3.516 \mathrm{E}+00$ & $1.113 \mathrm{E}+01$ & $2.372 \mathrm{E}+01$ & $2.050 \mathrm{E}-01$ & $1.116 \mathrm{E}-06$ & $4.410 \mathrm{E}-07$ \\
\hline GWO & $3.13 \mathrm{E}-230$ & $0.000 E+00$ & $2.600 \mathrm{E}+01$ & $9.075 \mathrm{E}-01$ & $4.999 \mathrm{E}-01$ & $3.280 \mathrm{E}-01$ \\
\hline GSA & $1.701 \mathrm{E}+00$ & $1.279 \mathrm{E}-01$ & $8.600 \mathrm{E}+03$ & $1.545 \mathrm{E}+03$ & $1.457 \mathrm{E}+01$ & $1.887 \mathrm{E}+00$ \\
\hline \multirow{2}{*}{ Algorithm } & \multicolumn{2}{|c|}{ F7 } & \multicolumn{2}{|c|}{ F8 } & \multicolumn{2}{|c|}{ F9 } \\
\hline & AVG & STD & AVG & STD & AVG & STD \\
\hline CPA & $2.706 E-05$ & $7.163 \mathrm{E}-05$ & $-1.257 E+04$ & $5.145 \mathrm{E}-12$ & $0.000 E+00$ & $0.000 E+00$ \\
\hline PSO & $1.130 \mathrm{E}+02$ & $2.615 \mathrm{E}+01$ & $-7.026 \mathrm{E}+03$ & $9.487 \mathrm{E}+02$ & $3.380 \mathrm{E}+02$ & $1.904 \mathrm{E}+01$ \\
\hline $\mathrm{DE}$ & $1.633 \mathrm{E}-03$ & $3.467 \mathrm{E}-04$ & $-1.245 \mathrm{E}+04$ & $1.190 \mathrm{E}+02$ & $1.327 \mathrm{E}-01$ & $3.440 \mathrm{E}-01$ \\
\hline $\mathrm{ABC}$ & $6.528 \mathrm{E}-02$ & $1.524 \mathrm{E}-02$ & $-1.257 E+04$ & $2.925 \mathrm{E}-12$ & $0.000 E+00$ & $0.000 \mathrm{E}+00$ \\
\hline SCA & $1.621 \mathrm{E}-03$ & $1.522 \mathrm{E}-03$ & $-4.481 \mathrm{E}+03$ & $2.686 \mathrm{E}+02$ & $0.000 E+00$ & $0.000 E+00$ \\
\hline SSA & $5.192 \mathrm{E}-03$ & $1.976 \mathrm{E}-03$ & $-7.686 \mathrm{E}+03$ & $6.040 \mathrm{E}+02$ & $6.978 \mathrm{E}+01$ & $1.821 \mathrm{E}+01$ \\
\hline BA & $1.400 \mathrm{E}+01$ & $1.200 \mathrm{E}+01$ & $-7.093 \mathrm{E}+03$ & $6.521 \mathrm{E}+02$ & $2.419 \mathrm{E}+02$ & $2.211 \mathrm{E}+01$ \\
\hline FA & $3.794 \mathrm{E}+00$ & $6.533 \mathrm{E}-01$ & $-4.212 \mathrm{E}+03$ & $2.135 \mathrm{E}+02$ & $2.239 \mathrm{E}+02$ & $1.247 \mathrm{E}+01$ \\
\hline MFO & $1.420 \mathrm{E}+00$ & $2.510 \mathrm{E}+00$ & $-8.692 \mathrm{E}+03$ & $9.020 \mathrm{E}+02$ & $1.543 \mathrm{E}+02$ & $4.011 \mathrm{E}+01$ \\
\hline WOA & $1.059 \mathrm{E}-04$ & $1.075 \mathrm{E}-04$ & $-1.228 \mathrm{E}+04$ & $7.883 \mathrm{E}+02$ & $0.000 E+00$ & $0.000 \mathrm{E}+00$ \\
\hline GWO & $3.734 \mathrm{E}-05$ & $2.772 E-05$ & $-6.223 \mathrm{E}+03$ & $6.377 \mathrm{E}+02$ & $0.000 E+00$ & $0.000 \mathrm{E}+00$ \\
\hline GSA & $2.970 \mathrm{E}+01$ & $4.650 \mathrm{E}+00$ & $-2.698 \mathrm{E}+03$ & $5.512 \mathrm{E}+02$ & $1.969 \mathrm{E}+02$ & $8.544 \mathrm{E}+00$ \\
\hline \multirow{2}{*}{ Algorithm } & \multicolumn{2}{|c|}{ F10 } & \multicolumn{2}{|c|}{ F11 } & \multicolumn{2}{|c|}{ F12 } \\
\hline & AVG & STD & AVG & STD & $\mathrm{AVG}$ & STD \\
\hline CPA & $8.882 E-16$ & $0.000 \mathrm{E}+00$ & $0.000 E+00$ & $0.000 E+00$ & $4.731 E-29$ & $1.215 \mathrm{E}-28$ \\
\hline PSO & $7.761 \mathrm{E}+00$ & $2.379 \mathrm{E}-01$ & $1.007 \mathrm{E}+00$ & $1.564 \mathrm{E}-02$ & $3.243 \mathrm{E}+00$ & $3.976 \mathrm{E}-01$ \\
\hline
\end{tabular}


Tu et al.: The Colony Predation Algorithm

\begin{tabular}{|c|c|c|c|c|c|c|}
\hline $\mathrm{DE}$ & $7.520 \mathrm{E}-15$ & $1.228 \mathrm{E}-15$ & $0.000 \mathrm{E}+00$ & $0.000 \mathrm{E}+00$ & $1.571 \mathrm{E}-32$ & $5.567 \mathrm{E}-48$ \\
\hline $\mathrm{ABC}$ & $3.784 \mathrm{E}-14$ & $3.178 \mathrm{E}-15$ & $1.110 \mathrm{E}-16$ & $1.010 \mathrm{E}-16$ & $4.685 \mathrm{E}-16$ & $5.305 \mathrm{E}-17$ \\
\hline SCA & $1.253 \mathrm{E}+01$ & $8.896 \mathrm{E}+00$ & $6.883 \mathrm{E}-04$ & $3.770 \mathrm{E}-03$ & $3.142 \mathrm{E}-01$ & $5.660 \mathrm{E}-02$ \\
\hline SSA & $1.708 \mathrm{E}+00$ & $7.414 \mathrm{E}-01$ & $1.370 \mathrm{E}-02$ & $1.151 \mathrm{E}-02$ & $9.638 \mathrm{E}-01$ & $1.748 \mathrm{E}+00$ \\
\hline BA & $1.766 \mathrm{E}+00$ & $7.075 \mathrm{E}-01$ & $1.381 \mathrm{E}-02$ & $1.907 \mathrm{E}-02$ & $7.716 \mathrm{E}+00$ & $3.099 \mathrm{E}+00$ \\
\hline FA & $1.583 \mathrm{E}+01$ & $2.980 \mathrm{E}-01$ & $1.006 \mathrm{E}+02$ & $1.008 \mathrm{E}+01$ & $1.854 \mathrm{E}+06$ & $8.197 \mathrm{E}+05$ \\
\hline MFO & $1.216 \mathrm{E}+01$ & $9.045 \mathrm{E}+00$ & $3.013 \mathrm{E}+01$ & $6.424 \mathrm{E}+01$ & $1.837 \mathrm{E}-01$ & $3.564 \mathrm{E}-01$ \\
\hline WOA & $2.783 \mathrm{E}-15$ & $2.030 \mathrm{E}-15$ & $0.000 E+00$ & $0.000 \mathrm{E}+00$ & $1.969 \mathrm{E}-07$ & $8.899 \mathrm{E}-08$ \\
\hline GWO & $7.401 \mathrm{E}-15$ & $1.347 \mathrm{E}-15$ & $0.000 \mathrm{E}+00$ & $0.000 \mathrm{E}+00$ & $2.672 \mathrm{E}-02$ & $1.114 \mathrm{E}-02$ \\
\hline GSA & $4.122 \mathrm{E}+00$ & $1.492 \mathrm{E}-01$ & $5.166 \mathrm{E}-01$ & $5.058 \mathrm{E}-02$ & $1.315 \mathrm{E}+00$ & $2.810 \mathrm{E}-01$ \\
\hline \multirow{2}{*}{ Algorithm } & \multicolumn{2}{|c|}{ F13 } & \multicolumn{2}{|c|}{$\mathrm{F} 14$} & \multicolumn{2}{|c|}{ F15 } \\
\hline & AVG & STD & AVG & STD & AVG & STD \\
\hline CPA & $5.489 \mathrm{E}-28$ & $5.171 \mathrm{E}-28$ & $9.980 \mathrm{E}-01$ & $1.304 E-16$ & $3.075 E-04$ & $7.282 \mathrm{E}-17$ \\
\hline PSO & $1.503 \mathrm{E}+01$ & $1.510 \mathrm{E}+00$ & $2.383 \mathrm{E}+00$ & $1.669 \mathrm{E}+00$ & $9.506 \mathrm{E}-04$ & $5.628 \mathrm{E}-05$ \\
\hline $\mathrm{DE}$ & $1.350 E-32$ & $5.567 E-48$ & $1.031 \mathrm{E}+00$ & $1.815 \mathrm{E}-01$ & $9.801 \mathrm{E}-04$ & $3.661 \mathrm{E}-03$ \\
\hline $\mathrm{ABC}$ & $4.594 \mathrm{E}-16$ & $6.662 \mathrm{E}-17$ & $9.980 \mathrm{E}-01$ & $0.000 \mathrm{E}+00$ & $4.034 \mathrm{E}-04$ & $7.263 \mathrm{E}-05$ \\
\hline SCA & $1.992 \mathrm{E}+00$ & $1.686 \mathrm{E}-01$ & $9.980 \mathrm{E}-01$ & $5.240 \mathrm{E}-08$ & $5.934 \mathrm{E}-04$ & $4.165 \mathrm{E}-04$ \\
\hline SSA & $4.761 \mathrm{E}-03$ & $5.538 \mathrm{E}-03$ & $9.980 \mathrm{E}-01$ & $2.020 \mathrm{E}-16$ & $5.191 \mathrm{E}-04$ & $3.684 \mathrm{E}-04$ \\
\hline $\mathrm{BA}$ & $1.373 \mathrm{E}-01$ & $9.713 \mathrm{E}-02$ & $2.614 \mathrm{E}+00$ & $1.687 \mathrm{E}+00$ & $3.487 \mathrm{E}-03$ & $6.736 \mathrm{E}-03$ \\
\hline FA & $1.456 \mathrm{E}+07$ & $4.711 \mathrm{E}+06$ & $9.980 \mathrm{E}-01$ & $6.804 \mathrm{E}-06$ & $9.327 \mathrm{E}-04$ & $1.455 \mathrm{E}-04$ \\
\hline MFO & $1.367 \mathrm{E}+07$ & $7.487 \mathrm{E}+07$ & $2.607 \mathrm{E}+00$ & $2.493 \mathrm{E}+00$ & $9.576 \mathrm{E}-04$ & $5.088 \mathrm{E}-04$ \\
\hline WOA & $3.696 \mathrm{E}-04$ & $2.006 \mathrm{E}-03$ & $9.980 E-01$ & $6.331 \mathrm{E}-15$ & $4.921 \mathrm{E}-04$ & $3.719 \mathrm{E}-04$ \\
\hline GWO & $4.094 \mathrm{E}-01$ & $1.845 \mathrm{E}-01$ & $3.546 \mathrm{E}+00$ & $3.765 \mathrm{E}+00$ & $4.410 \mathrm{E}-03$ & $8.118 \mathrm{E}-03$ \\
\hline GSA & $7.866 \mathrm{E}+00$ & $1.265 \mathrm{E}+00$ & $9.980 \mathrm{E}-01$ & $1.400 \mathrm{E}-05$ & $1.048 \mathrm{E}-03$ & $2.185 \mathrm{E}-04$ \\
\hline \multirow{2}{*}{ Algorithm } & \multicolumn{2}{|c|}{ F16 } & \multicolumn{2}{|c|}{ F17 } & \multicolumn{2}{|c|}{ F18 } \\
\hline & AVG & STD & AVG & STD & AVG & STD \\
\hline CPA & $-1.032 E+00$ & $5.831 \mathrm{E}-16$ & $3.979 E-01$ & $0.000 \mathrm{E}+00$ & $3.000 \mathrm{E}+00$ & $1.072 E-15$ \\
\hline PSO & $-1.032 E+00$ & $8.495 \mathrm{E}-05$ & $3.979 \mathrm{E}-01$ & $3.811 \mathrm{E}-05$ & $3.006 \mathrm{E}+00$ & $5.354 \mathrm{E}-03$ \\
\hline $\mathrm{DE}$ & $-1.032 \mathrm{E}+00$ & $6.775 \mathrm{E}-16$ & $3.979 E-01$ & $0.000 \mathrm{E}+00$ & $3.000 \mathrm{E}+00$ & $1.951 \mathrm{E}-15$ \\
\hline $\mathrm{ABC}$ & $-1.032 E+00$ & $6.775 \mathrm{E}-16$ & $3.979 \mathrm{E}-01$ & $0.000 E+00$ & $3.000 \mathrm{E}+00$ & $6.303 \mathrm{E}-07$ \\
\hline SCA & $-1.032 \mathrm{E}+00$ & $1.771 \mathrm{E}-06$ & $3.979 \mathrm{E}-01$ & $4.054 \mathrm{E}-05$ & $3.000 \mathrm{E}+00$ & $1.129 \mathrm{E}-07$ \\
\hline SSA & $-1.032 E+00$ & $4.225 \mathrm{E}-16$ & $3.979 \mathrm{E}-01$ & $0.000 E+00$ & $3.000 \mathrm{E}+00$ & $1.020 \mathrm{E}-14$ \\
\hline BA & $-1.032 E+00$ & $4.922 \mathrm{E}-05$ & $3.979 \mathrm{E}-01$ & $3.706 \mathrm{E}-05$ & $3.005 \mathrm{E}+00$ & $5.127 \mathrm{E}-03$ \\
\hline FA & $-1.032 E+00$ & $8.254 \mathrm{E}-05$ & $3.979 \mathrm{E}-01$ & $3.534 \mathrm{E}-05$ & $3.001 \mathrm{E}+00$ & $7.757 \mathrm{E}-04$ \\
\hline MFO & $-1.032 E+00$ & $6.775 \mathrm{E}-16$ & $3.979 E-01$ & $0.000 \mathrm{E}+00$ & $3.000 \mathrm{E}+00$ & $1.424 \mathrm{E}-15$ \\
\hline WOA & $-1.032 E+00$ & $1.086 \mathrm{E}-15$ & $3.979 \mathrm{E}-01$ & $3.636 \mathrm{E}-11$ & $3.000 \mathrm{E}+00$ & $3.758 \mathrm{E}-08$ \\
\hline GWO & $-1.032 E+00$ & $1.368 \mathrm{E}-11$ & $3.979 E-01$ & $5.214 \mathrm{E}-10$ & $3.000 \mathrm{E}+00$ & $4.630 \mathrm{E}-08$ \\
\hline GSA & $-1.031 \mathrm{E}+00$ & $1.454 \mathrm{E}-04$ & $3.981 \mathrm{E}-01$ & $1.345 \mathrm{E}-04$ & $3.005 \mathrm{E}+00$ & $5.753 \mathrm{E}-03$ \\
\hline \multirow{2}{*}{ Algorithm } & \multicolumn{2}{|c|}{ F19 } & \multicolumn{2}{|c|}{ F20 } & \multicolumn{2}{|c|}{ F21 } \\
\hline & AVG & STD & $\mathrm{AVG}$ & STD & AVG & STD \\
\hline CPA & $-3.863 E+00$ & $2.518 \mathrm{E}-15$ & $-3.322 \mathrm{E}+00$ & $1.477 \mathrm{E}-15$ & $-8.964 \mathrm{E}+00$ & $2.193 \mathrm{E}+00$ \\
\hline PSO & $-3.857 \mathrm{E}+00$ & $3.329 \mathrm{E}-03$ & $-2.980 \mathrm{E}+00$ & $1.751 \mathrm{E}-01$ & $-7.350 \mathrm{E}+00$ & $1.315 \mathrm{E}+00$ \\
\hline $\mathrm{DE}$ & $-3.863 E+00$ & $2.710 \mathrm{E}-15$ & $-3.317 \mathrm{E}+00$ & $1.972 \mathrm{E}-02$ & $-9.648 \mathrm{E}+00$ & $1.542 \mathrm{E}+00$ \\
\hline $\mathrm{ABC}$ & $-3.863 E+00$ & $2.372 E-15$ & $-3.322 \mathrm{E}+00$ & $1.355 \mathrm{E}-15$ & $-1.015 \mathrm{E}+01$ & $5.761 \mathrm{E}-15$ \\
\hline SCA & $-3.855 \mathrm{E}+00$ & $1.983 \mathrm{E}-03$ & $-2.867 \mathrm{E}+00$ & $4.086 \mathrm{E}-01$ & $-2.571 \mathrm{E}+00$ & $2.466 \mathrm{E}+00$ \\
\hline SSA & $-3.863 \mathrm{E}+00$ & $1.820 \mathrm{E}-15$ & $-3.235 \mathrm{E}+00$ & $5.348 \mathrm{E}-02$ & $-9.816 E+00$ & $1.282 \mathrm{E}+00$ \\
\hline $\mathrm{BA}$ & $-3.855 \mathrm{E}+00$ & $2.919 \mathrm{E}-03$ & $-3.056 \mathrm{E}+00$ & $4.663 \mathrm{E}-02$ & $-8.865 \mathrm{E}+00$ & $1.598 \mathrm{E}+00$ \\
\hline FA & $-3.862 \mathrm{E}+00$ & $2.164 \mathrm{E}-04$ & $-3.258 \mathrm{E}+00$ & $4.680 \mathrm{E}-02$ & $-8.419 \mathrm{E}+00$ & $6.918 \mathrm{E}-01$ \\
\hline
\end{tabular}


Journal of Bionic Engineering (2021) Vol.18 No.3

\begin{tabular}{|c|c|c|c|c|c|c|}
\hline MFO & $-3.863 \mathrm{E}+00$ & $2.710 \mathrm{E}-15$ & $-3.226 \mathrm{E}+00$ & $5.648 \mathrm{E}-02$ & $-5.307 \mathrm{E}+00$ & $3.343 \mathrm{E}+00$ \\
\hline WOA & $-3.862 \mathrm{E}+00$ & $1.999 \mathrm{E}-03$ & $-3.220 \mathrm{E}+00$ & $1.768 \mathrm{Ev} 01$ & $-1.015 \mathrm{E}+01$ & $1.825 \mathrm{E}-07$ \\
\hline GWO & $-3.863 \mathrm{E}+00$ & $1.439 \mathrm{E}-03$ & $-3.253 \mathrm{E}+00$ & $7.313 \mathrm{E}-02$ & $-9.816 E+00$ & $1.282 \mathrm{E}+00$ \\
\hline GSA & $-3.862 \mathrm{E}+00$ & $6.067 \mathrm{E}-04$ & $-3.204 \mathrm{E}+00$ & $4.201 \mathrm{E}-02$ & $-6.517 \mathrm{E}+00$ & $1.193 \mathrm{E}+00$ \\
\hline \multirow{2}{*}{ Algorithm } & \multicolumn{2}{|c|}{ F22 } & \multicolumn{2}{|c|}{ F23 } & \multicolumn{2}{|c|}{ F24 } \\
\hline & AVG & STD & AVG & STD & AVG & STD \\
\hline CPA & $-8.277 \mathrm{E}+00$ & $2.648 \mathrm{E}+00$ & $-8.373 \mathrm{E}+00$ & $2.695 \mathrm{E}+00$ & $3.676 E+05$ & $2.006 E+05$ \\
\hline PSO & $-7.875 \mathrm{E}+00$ & $8.814 \mathrm{E}-01$ & $-8.148 \mathrm{E}+00$ & $1.007 \mathrm{E}+00$ & $7.374 \mathrm{E}+06$ & $1.908 \mathrm{E}+06$ \\
\hline $\mathrm{DE}$ & $-1.040 \mathrm{E}+01$ & $1.807 \mathrm{E}-15$ & $-1.054 \mathrm{E}+01$ & $1.616 \mathrm{E}-15$ & $1.596 \mathrm{E}+07$ & $4.997 \mathrm{E}+06$ \\
\hline $\mathrm{ABC}$ & $-1.040 \mathrm{E}+01$ & $3.299 \mathrm{E}-16$ & $-1.054 \mathrm{E}+01$ & $1.582 \mathrm{E}-15$ & $3.766 \mathrm{E}+06$ & $1.535 \mathrm{E}+06$ \\
\hline SCA & $-3.951 \mathrm{E}+00$ & $2.804 \mathrm{E}+00$ & $-6.140 \mathrm{E}+00$ & $2.222 \mathrm{E}+00$ & $2.094 \mathrm{E}+08$ & $7.040 \mathrm{E}+07$ \\
\hline SSA & $-9.876 \mathrm{E}+00$ & $1.609 \mathrm{E}+00$ & $-1.054 \mathrm{E}+01$ & $1.096 \mathrm{E}-12$ & $1.067 \mathrm{E}+06$ & $4.225 \mathrm{E}+05$ \\
\hline BA & $-8.609 \mathrm{E}+00$ & $2.074 \mathrm{E}+00$ & $-9.336 \mathrm{E}+00$ & $1.534 \mathrm{E}+00$ & $6.156 \mathrm{E}+05$ & $2.036 \mathrm{E}+05$ \\
\hline FA & $-8.824 \mathrm{E}+00$ & $7.890 \mathrm{E}-01$ & $-8.854 \mathrm{E}+00$ & $8.084 \mathrm{E}-01$ & $2.314 \mathrm{E}+08$ & $4.509 \mathrm{E}+07$ \\
\hline MFO & $-8.076 \mathrm{E}+00$ & $3.391 \mathrm{E}+00$ & $-7.800 \mathrm{E}+00$ & $3.704 \mathrm{E}+00$ & $1.269 \mathrm{E}+08$ & $1.543 \mathrm{E}+08$ \\
\hline WOA & $-1.040 \mathrm{E}+01$ & $1.062 \mathrm{E}-07$ & $-1.054 \mathrm{E}+01$ & $2.224 E-07$ & $2.423 \mathrm{E}+07$ & $1.115 \mathrm{E}+07$ \\
\hline GWO & $-1.040 \mathrm{E}+01$ & $4.830 \mathrm{E}-07$ & $-1.054 \mathrm{E}+01$ & $4.154 \mathrm{E}-07$ & $6.205 \mathrm{E}+07$ & $5.313 \mathrm{E}+07$ \\
\hline GSA & $-7.245 \mathrm{E}+00$ & $1.340 \mathrm{E}+00$ & $-6.988 \mathrm{E}+00$ & $1.215 \mathrm{E}+00$ & $1.340 \mathrm{E}+06$ & $3.315 \mathrm{E}+05$ \\
\hline \multirow{2}{*}{ Algorithm } & \multicolumn{2}{|c|}{$\mathrm{F} 25$} & \multicolumn{2}{|c|}{ F26 } & \multicolumn{2}{|c|}{ F27 } \\
\hline & AVG & STD & AVG & STD & AVG & STD \\
\hline CPA & $1.535 \mathrm{E}+04$ & $1.213 \mathrm{E}+04$ & $3.445 \mathrm{E}+02$ & $3.178 \mathrm{E}+01$ & $4.531 \mathrm{E}+02$ & $3.600 \mathrm{E}+01$ \\
\hline PSO & $1.418 \mathrm{E}+08$ & $1.160 \mathrm{E}+07$ & $7.824 \mathrm{E}+02$ & $6.845 \mathrm{E}+01$ & $4.679 \mathrm{E}+02$ & $3.958 \mathrm{E}+01$ \\
\hline $\mathrm{DE}$ & $6.111 \mathrm{E}+02$ & $1.444 \mathrm{E}+03$ & $3.211 \mathrm{E}+02$ & $1.978 \mathrm{E}+01$ & $4.819 \mathrm{E}+02$ & $3.774 \mathrm{E}+01$ \\
\hline $\mathrm{ABC}$ & $4.114 \mathrm{E}+02$ & $2.065 \mathrm{E}+02$ & $9.500 \mathrm{E}+02$ & $6.334 \mathrm{E}+02$ & $4.214 \mathrm{E}+02$ & $2.895 E+01$ \\
\hline SCA & $1.481 \mathrm{E}+10$ & $3.069 \mathrm{E}+09$ & $3.652 \mathrm{E}+04$ & $6.005 \mathrm{E}+03$ & $1.318 \mathrm{E}+03$ & $2.116 \mathrm{E}+02$ \\
\hline SSA & $1.367 \mathrm{E}+04$ & $1.154 \mathrm{E}+04$ & $3.751 \mathrm{E}+02$ & $6.054 \mathrm{E}+01$ & $4.723 \mathrm{E}+02$ & $4.069 \mathrm{E}+01$ \\
\hline $\mathrm{BA}$ & $5.351 \mathrm{E}+05$ & $3.265 \mathrm{E}+05$ & $3.076 \mathrm{E}+02$ & $1.270 \mathrm{E}+01$ & $4.303 \mathrm{E}+02$ & $4.287 \mathrm{E}+01$ \\
\hline FA & $1.473 \mathrm{E}+10$ & $1.261 \mathrm{E}+09$ & $5.965 \mathrm{E}+04$ & $8.090 \mathrm{E}+03$ & $1.520 \mathrm{E}+03$ & $1.316 \mathrm{E}+02$ \\
\hline MFO & $1.226 \mathrm{E}+10$ & $8.622 \mathrm{E}+09$ & $1.128 \mathrm{E}+05$ & $5.419 \mathrm{E}+04$ & $1.575 \mathrm{E}+03$ & $1.413 \mathrm{E}+03$ \\
\hline WOA & $8.920 \mathrm{E}+05$ & $1.167 \mathrm{E}+06$ & $2.577 \mathrm{E}+04$ & $2.184 \mathrm{E}+04$ & $5.662 \mathrm{E}+02$ & $5.067 \mathrm{E}+01$ \\
\hline GWO & $2.474 \mathrm{E}+09$ & $2.128 \mathrm{E}+09$ & $2.923 \mathrm{E}+04$ & $9.607 \mathrm{E}+03$ & $6.344 \mathrm{E}+02$ & $8.910 \mathrm{E}+01$ \\
\hline GSA & $2.020 \mathrm{E}+07$ & $2.773 \mathrm{E}+06$ & $7.236 \mathrm{E}+03$ & $3.532 \mathrm{E}+03$ & $4.598 \mathrm{E}+02$ & $6.239 \mathrm{E}+01$ \\
\hline \multirow{2}{*}{ Algorithm } & \multicolumn{2}{|c|}{ F28 } & \multicolumn{2}{|c|}{ F29 } & \multicolumn{2}{|c|}{$\mathrm{F} 30$} \\
\hline & AVG & STD & AVG & STD & AVG & STD \\
\hline CPA & $5.200 \mathrm{E}+02$ & $1.629 E-04$ & $6.171 \mathrm{E}+02$ & $3.542 \mathrm{E}+00$ & $7.000 E+02$ & $3.145 \mathrm{E}-02$ \\
\hline PSO & $5.209 \mathrm{E}+02$ & $5.256 \mathrm{E}-02$ & $6.227 \mathrm{E}+02$ & $2.204 \mathrm{E}+00$ & $7.022 \mathrm{E}+02$ & $1.224 \mathrm{E}-01$ \\
\hline $\mathrm{DE}$ & $5.205 \mathrm{E}+02$ & $5.177 \mathrm{E}-02$ & $6.176 \mathrm{E}+02$ & $2.677 \mathrm{E}+00$ & $7.000 E+02$ & $1.996 \mathrm{E}-04$ \\
\hline $\mathrm{ABC}$ & $5.202 \mathrm{E}+02$ & $2.880 \mathrm{E}-02$ & $6.141 E+02$ & $1.788 \mathrm{E}+00$ & $7.000 E+02$ & $5.517 E-09$ \\
\hline SCA & $5.209 \mathrm{E}+02$ & $4.492 \mathrm{E}-02$ & $6.334 \mathrm{E}+02$ & $2.356 \mathrm{E}+00$ & $8.308 \mathrm{E}+02$ & $2.452 \mathrm{E}+01$ \\
\hline SSA & $5.201 \mathrm{E}+02$ & $8.675 \mathrm{E}-02$ & $6.175 \mathrm{E}+02$ & $4.407 \mathrm{E}+00$ & $7.000 E+02$ & $1.360 \mathrm{E}-02$ \\
\hline $\mathrm{BA}$ & $5.210 \mathrm{E}+02$ & $4.440 \mathrm{E}-02$ & $6.339 \mathrm{E}+02$ & $2.557 \mathrm{E}+00$ & $7.006 \mathrm{E}+02$ & $2.036 \mathrm{E}-01$ \\
\hline FA & $5.209 \mathrm{E}+02$ & $6.136 \mathrm{E}-02$ & $6.332 \mathrm{E}+02$ & $1.101 \mathrm{E}+00$ & $8.278 \mathrm{E}+02$ & $1.380 \mathrm{E}+01$ \\
\hline MFO & $5.203 \mathrm{E}+02$ & $1.763 \mathrm{E}-01$ & $6.249 \mathrm{E}+02$ & $3.783 \mathrm{E}+00$ & $8.315 \mathrm{E}+02$ & $7.851 \mathrm{E}+01$ \\
\hline WOA & $5.203 \mathrm{E}+02$ & $2.092 \mathrm{E}-01$ & $6.351 \mathrm{E}+02$ & $3.898 \mathrm{E}+00$ & $7.008 \mathrm{E}+02$ & $1.289 \mathrm{E}-01$ \\
\hline GWO & $5.209 \mathrm{E}+02$ & $3.988 \mathrm{E}-02$ & $6.130 \mathrm{E}+02$ & $3.038 \mathrm{E}+00$ & $7.159 \mathrm{E}+02$ & $1.547 \mathrm{E}+01$ \\
\hline GSA & $5.209 \mathrm{E}+02$ & $4.954 \mathrm{E}-02$ & $6.074 \mathrm{E}+02$ & $2.053 \mathrm{E}+00$ & $7.012 \mathrm{E}+02$ & $1.918 \mathrm{E}-02$ \\
\hline \multirow{2}{*}{ Algorithm } & \multicolumn{2}{|c|}{ F31 } & \multicolumn{2}{|c|}{ F32 } & \multicolumn{2}{|c|}{$\mathrm{F} 33$} \\
\hline & AVG & STD & AVG & STD & AVG & STD \\
\hline
\end{tabular}


Tu et al.: The Colony Predation Algorithm

\begin{tabular}{|c|c|c|c|c|c|c|}
\hline CPA & $8.000 E+02$ & $2.704 E-13$ & $1.018 \mathrm{E}+03$ & $2.447 \mathrm{E}+01$ & $1.001 \mathrm{E}+03$ & $1.312 \mathrm{E}+00$ \\
\hline PSO & $9.669 \mathrm{E}+02$ & $2.246 \mathrm{E}+01$ & $1.101 \mathrm{E}+03$ & $1.854 \mathrm{E}+01$ & $5.009 \mathrm{E}+03$ & $4.493 \mathrm{E}+02$ \\
\hline $\mathrm{DE}$ & $8.006 \mathrm{E}+02$ & $6.744 \mathrm{E}-01$ & $1.001 \mathrm{E}+03$ & $7.356 \mathrm{E}+00$ & $1.021 \mathrm{E}+03$ & $2.113 \mathrm{E}+01$ \\
\hline $\mathrm{ABC}$ & $8.000 \mathrm{E}+02$ & $4.222 \mathrm{E}-14$ & $9.800 \mathrm{E}+02$ & $1.082 \mathrm{E}+01$ & $1.000 \mathrm{E}+03$ & $4.099 \mathrm{E}-02$ \\
\hline SCA & $1.035 \mathrm{E}+03$ & $2.005 \mathrm{E}+01$ & $1.166 \mathrm{E}+03$ & $1.653 \mathrm{E}+01$ & $7.044 \mathrm{E}+03$ & $5.401 \mathrm{E}+02$ \\
\hline SSA & $8.998 \mathrm{E}+02$ & $3.009 \mathrm{E}+01$ & $1.011 \mathrm{E}+03$ & $3.124 \mathrm{E}+01$ & $4.490 \mathrm{E}+03$ & $5.899 \mathrm{E}+02$ \\
\hline $\mathrm{BA}$ & $1.024 \mathrm{E}+03$ & $5.267 \mathrm{E}+01$ & $1.183 \mathrm{E}+03$ & $5.824 \mathrm{E}+01$ & $5.323 \mathrm{E}+03$ & $6.745 \mathrm{E}+02$ \\
\hline FA & $1.023 \mathrm{E}+03$ & $9.813 \mathrm{E}+00$ & $1.154 \mathrm{E}+03$ & $1.127 \mathrm{E}+01$ & $7.403 \mathrm{E}+03$ & $2.741 \mathrm{E}+02$ \\
\hline MFO & $9.387 \mathrm{E}+02$ & $4.607 \mathrm{E}+01$ & $1.131 \mathrm{E}+03$ & $5.231 \mathrm{E}+01$ & $4.435 \mathrm{E}+03$ & $8.969 \mathrm{E}+02$ \\
\hline WOA & $9.898 \mathrm{E}+02$ & $4.339 \mathrm{E}+01$ & $1.130 \mathrm{E}+03$ & $6.215 \mathrm{E}+01$ & $4.706 \mathrm{E}+03$ & $7.236 \mathrm{E}+02$ \\
\hline GWO & $8.822 \mathrm{E}+02$ & $1.641 \mathrm{E}+01$ & $9.884 \mathrm{E}+02$ & $1.553 \mathrm{E}+01$ & $3.254 \mathrm{E}+03$ & $4.993 \mathrm{E}+02$ \\
\hline GSA & $8.288 \mathrm{E}+02$ & $4.527 \mathrm{E}+00$ & $9.538 E+02$ & $1.094 \mathrm{E}+01$ & $2.182 \mathrm{E}+03$ & $2.591 \mathrm{E}+02$ \\
\hline \multirow{2}{*}{ Algorithm } & \multicolumn{2}{|c|}{ F34 } & \multicolumn{2}{|c|}{ F35 } & \multicolumn{2}{|c|}{ F36 } \\
\hline & AVG & STD & AVG & STD & AVG & STD \\
\hline CPA & $3.636 \mathrm{E}+03$ & $5.443 \mathrm{E}+02$ & $1.200 \mathrm{E}+03$ & $5.158 \mathrm{E}-02$ & $1.300 \mathrm{E}+03$ & $1.340 \mathrm{E}-01$ \\
\hline PSO & $5.688 \mathrm{E}+03$ & $5.053 \mathrm{E}+02$ & $1.202 \mathrm{E}+03$ & $2.961 \mathrm{E}-01$ & $1.300 \mathrm{E}+03$ & $6.230 \mathrm{E}-02$ \\
\hline $\mathrm{DE}$ & $5.472 \mathrm{E}+03$ & $3.090 \mathrm{E}+02$ & $1.201 \mathrm{E}+03$ & $9.450 \mathrm{E}-02$ & $1.300 \mathrm{E}+03$ & $4.972 \mathrm{E}-02$ \\
\hline $\mathrm{AB}$ & $3.039 \mathrm{E}+03$ & $2.157 E+02$ & $1.200 \mathrm{E}+03$ & $2.989 \mathrm{E}-02$ & $1.300 \mathrm{E}+03$ & $2.532 \mathrm{E}-02$ \\
\hline SCA & $7.931 \mathrm{E}+03$ & $3.709 \mathrm{E}+02$ & $1.202 \mathrm{E}+03$ & $2.384 \mathrm{E}-01$ & $1.303 \mathrm{E}+03$ & $2.845 \mathrm{E}-01$ \\
\hline SSA & $4.787 \mathrm{E}+03$ & $8.046 \mathrm{E}+02$ & $1.200 \mathrm{E}+03$ & $1.995 \mathrm{E}-01$ & $1.301 \mathrm{E}+03$ & $1.089 \mathrm{E}-01$ \\
\hline BA & $5.527 \mathrm{E}+03$ & $5.064 \mathrm{E}+02$ & $1.201 \mathrm{E}+03$ & $3.595 \mathrm{E}-01$ & $1.301 \mathrm{E}+03$ & $1.260 \mathrm{E}-01$ \\
\hline FA & $7.825 \mathrm{E}+03$ & $2.862 \mathrm{E}+02$ & $1.202 \mathrm{E}+03$ & $2.438 \mathrm{E}-01$ & $1.303 \mathrm{E}+03$ & $2.081 \mathrm{E}-01$ \\
\hline MFO & $5.341 \mathrm{E}+03$ & $8.799 \mathrm{E}+02$ & $1.201 \mathrm{E}+03$ & $2.318 \mathrm{E}-01$ & $1.302 \mathrm{E}+03$ & $1.221 \mathrm{E}+00$ \\
\hline WOA & $5.555 \mathrm{E}+03$ & $6.909 \mathrm{E}+02$ & $1.202 \mathrm{E}+03$ & $3.954 \mathrm{E}-01$ & $1.300 \mathrm{E}+03$ & $9.840 \mathrm{E}-02$ \\
\hline GWO & $3.978 \mathrm{E}+03$ & $5.592 \mathrm{E}+02$ & $1.202 \mathrm{E}+03$ & $1.063 \mathrm{E}+00$ & $1.300 \mathrm{E}+03$ & $5.914 \mathrm{E}-02$ \\
\hline GSA & $2.771 E+03$ & $3.121 \mathrm{E}+02$ & $1.201 \mathrm{E}+03$ & $8.975 \mathrm{E}-02$ & $1.300 \mathrm{E}+03$ & $1.705 \mathrm{E}-02$ \\
\hline \multirow{2}{*}{ Algorithm } & \multicolumn{2}{|c|}{ F37 } & \multicolumn{2}{|c|}{ F38 } & \multicolumn{2}{|c|}{ F39 } \\
\hline & AVG & STD & AVG & STD & AVG & STD \\
\hline CPA & $1.400 \mathrm{E}+03$ & $1.023 \mathrm{E}-01$ & $1.512 \mathrm{E}+03$ & $6.941 \mathrm{E}+00$ & $1.610 \mathrm{E}+03$ & $6.793 \mathrm{E}-01$ \\
\hline PSO & $1.400 E+03$ & $1.412 \mathrm{E}-01$ & $1.516 \mathrm{E}+03$ & $1.839 \mathrm{E}+00$ & $1.612 \mathrm{E}+03$ & $4.881 \mathrm{E}-01$ \\
\hline $\mathrm{DE}$ & $1.400 \mathrm{E}+03$ & $6.446 \mathrm{E}-02$ & $1.511 \mathrm{E}+03$ & $6.473 E-01$ & $1.611 \mathrm{E}+03$ & $2.497 \mathrm{E}-01$ \\
\hline $\mathrm{ABC}$ & $1.400 E+03$ & $1.373 E-02$ & $1.507 \mathrm{E}+03$ & $1.170 \mathrm{E}+00$ & $1.610 \mathrm{E}+03$ & $2.602 \mathrm{E}-01$ \\
\hline SCA & $1.442 \mathrm{E}+03$ & $7.826 \mathrm{E}+00$ & $3.961 \mathrm{E}+03$ & $2.982 \mathrm{E}+03$ & $1.613 \mathrm{E}+03$ & $2.120 \mathrm{E}-01$ \\
\hline SSA & $1.400 E+03$ & $1.543 \mathrm{E}-01$ & $1.507 E+03$ & $2.483 \mathrm{E}+00$ & $1.611 \mathrm{E}+03$ & $7.004 \mathrm{E}-01$ \\
\hline BA & $1.400 E+03$ & $1.180 \mathrm{E}-01$ & $1.529 \mathrm{E}+03$ & $8.778 \mathrm{E}+00$ & $1.613 \mathrm{E}+03$ & $2.613 \mathrm{E}-01$ \\
\hline FA & $1.439 \mathrm{E}+03$ & $4.683 \mathrm{E}+00$ & $1.259 \mathrm{E}+04$ & $5.164 \mathrm{E}+03$ & $1.613 \mathrm{E}+03$ & $1.799 \mathrm{E}-01$ \\
\hline MFO & $1.433 \mathrm{E}+03$ & $1.741 \mathrm{E}+01$ & $2.410 \mathrm{E}+05$ & $4.907 \mathrm{E}+05$ & $1.613 \mathrm{E}+03$ & $6.215 \mathrm{E}-01$ \\
\hline WOA & $1.400 \mathrm{E}+03$ & $4.385 \mathrm{E}-02$ & $1.569 \mathrm{E}+03$ & $3.012 \mathrm{E}+01$ & $1.613 \mathrm{E}+03$ & $4.564 \mathrm{E}-01$ \\
\hline GWO & $1.405 \mathrm{E}+03$ & $7.487 \mathrm{E}+00$ & $1.740 \mathrm{E}+03$ & $6.094 \mathrm{E}+02$ & $1.611 \mathrm{E}+03$ & $6.985 \mathrm{E}-01$ \\
\hline GSA & $1.400 \mathrm{E}+03$ & $4.667 \mathrm{E}-02$ & $1.513 \mathrm{E}+03$ & $8.072 \mathrm{E}-01$ & $1.613 \mathrm{E}+03$ & $3.080 \mathrm{E}-01$ \\
\hline \multirow{2}{*}{ Algorithm } & \multicolumn{2}{|c|}{ F40 } & \multicolumn{2}{|c|}{ F41 } & \multicolumn{2}{|c|}{ F42 } \\
\hline & AVG & STD & AVG & STD & AVG & STD \\
\hline CPA & $4.160 \mathrm{E}+04$ & $1.816 \mathrm{E}+04$ & $3.910 \mathrm{E}+03$ & $2.657 \mathrm{E}+03$ & $1.910 \mathrm{E}+03$ & $1.087 \mathrm{E}+01$ \\
\hline PSO & $2.432 \mathrm{E}+05$ & $1.247 \mathrm{E}+05$ & $1.793 \mathrm{E}+06$ & $6.536 \mathrm{E}+05$ & $1.918 \mathrm{E}+03$ & $1.196 \mathrm{E}+01$ \\
\hline $\mathrm{DE}$ & $1.281 \mathrm{E}+06$ & $5.998 \mathrm{E}+05$ & $6.016 \mathrm{E}+03$ & $2.668 \mathrm{E}+03$ & $1.908 \mathrm{E}+03$ & $5.672 E-01$ \\
\hline $\mathrm{ABC}$ & $2.233 \mathrm{E}+06$ & $5.998 \mathrm{E}+05$ & $2.228 \mathrm{E}+03$ & $2.240 \mathrm{E}+02$ & $1.907 E+03$ & $8.408 \mathrm{E}-01$ \\
\hline SCA & $6.030 \mathrm{E}+06$ & $2.568 \mathrm{E}+06$ & $1.403 \mathrm{E}+08$ & $7.701 \mathrm{E}+07$ & $1.985 \mathrm{E}+03$ & $1.824 \mathrm{E}+01$ \\
\hline SSA & $7.223 \mathrm{E}+04$ & $5.276 \mathrm{E}+04$ & $6.367 \mathrm{E}+03$ & $5.054 \mathrm{E}+03$ & $1.914 \mathrm{E}+03$ & $2.056 \mathrm{E}+00$ \\
\hline
\end{tabular}


Journal of Bionic Engineering (2021) Vol.18 No.3

\begin{tabular}{|c|c|c|c|c|c|c|}
\hline BA & $6.341 \mathrm{E}+04$ & $3.716 \mathrm{E}+04$ & $8.157 \mathrm{E}+04$ & $4.387 \mathrm{E}+04$ & $1.920 \mathrm{E}+03$ & $1.853 \mathrm{E}+01$ \\
\hline FA & $6.328 \mathrm{E}+06$ & $1.858 \mathrm{E}+06$ & $2.608 \mathrm{E}+08$ & $6.795 \mathrm{E}+07$ & $2.004 \mathrm{E}+03$ & $1.392 \mathrm{E}+01$ \\
\hline MFO & $3.886 \mathrm{E}+06$ & $6.208 \mathrm{E}+06$ & $7.473 \mathrm{E}+07$ & $3.174 \mathrm{E}+08$ & $1.957 \mathrm{E}+03$ & $4.990 \mathrm{E}+01$ \\
\hline WOA & $3.975 \mathrm{E}+06$ & $1.973 \mathrm{E}+06$ & $6.768 \mathrm{E}+03$ & $4.935 \mathrm{E}+03$ & $1.942 \mathrm{E}+03$ & $3.425 \mathrm{E}+01$ \\
\hline GWO & $1.754 \mathrm{E}+06$ & $2.386 \mathrm{E}+06$ & $7.359 \mathrm{E}+06$ & $2.367 \mathrm{E}+07$ & $1.943 \mathrm{E}+03$ & $2.654 \mathrm{E}+01$ \\
\hline GSA & $1.622 \mathrm{E}+05$ & $1.090 \mathrm{E}+05$ & $6.460 \mathrm{E}+04$ & $2.087 \mathrm{E}+04$ & $1.907 \mathrm{E}+03$ & $6.565 \mathrm{E}-01$ \\
\hline \multirow{2}{*}{ Algorithm } & \multicolumn{2}{|c|}{ F43 } & \multicolumn{2}{|c|}{ F44 } & \multicolumn{2}{|c|}{ F45 } \\
\hline & AVG & STD & AVG & STD & AVG & STD \\
\hline CPA & $2.067 E+03$ & $2.333 E+01$ & $4.546 \mathrm{E}+04$ & $2.757 \mathrm{E}+04$ & $2.668 \mathrm{E}+03$ & $1.615 \mathrm{E}+02$ \\
\hline PSO & $2.306 \mathrm{E}+03$ & $8.243 \mathrm{E}+01$ & $8.730 \mathrm{E}+04$ & $4.722 \mathrm{E}+04$ & $2.888 \mathrm{E}+03$ & $2.124 \mathrm{E}+02$ \\
\hline $\mathrm{DE}$ & $3.960 \mathrm{E}+03$ & $1.133 \mathrm{E}+03$ & $2.277 \mathrm{E}+05$ & $1.048 \mathrm{E}+05$ & $2.359 E+03$ & $8.172 E+01$ \\
\hline $\mathrm{ABC}$ & $7.245 \mathrm{E}+03$ & $2.228 \mathrm{E}+03$ & $1.901 \mathrm{E}+05$ & $9.684 \mathrm{E}+04$ & $2.451 \mathrm{E}+03$ & $9.647 \mathrm{E}+01$ \\
\hline SCA & $1.363 \mathrm{E}+04$ & $3.773 \mathrm{E}+03$ & $1.062 \mathrm{E}+06$ & $5.377 \mathrm{E}+05$ & $2.935 \mathrm{E}+03$ & $1.330 \mathrm{E}+02$ \\
\hline SSA & $2.301 \mathrm{E}+03$ & $9.297 \mathrm{E}+01$ & $5.301 \mathrm{E}+04$ & $2.962 \mathrm{E}+04$ & $2.586 \mathrm{E}+03$ & $1.856 \mathrm{E}+02$ \\
\hline BA & $2.348 \mathrm{E}+03$ & $8.760 \mathrm{E}+01$ & $4.187 E+04$ & $2.114 E+04$ & $3.253 \mathrm{E}+03$ & $2.515 \mathrm{E}+02$ \\
\hline FA & $1.896 \mathrm{E}+04$ & $6.842 \mathrm{E}+03$ & $1.577 \mathrm{E}+06$ & $6.740 \mathrm{E}+05$ & $2.914 \mathrm{E}+03$ & $1.173 \mathrm{E}+02$ \\
\hline MFO & $7.073 \mathrm{E}+04$ & $7.495 \mathrm{E}+04$ & $8.311 \mathrm{E}+05$ & $1.885 \mathrm{E}+06$ & $2.999 \mathrm{E}+03$ & $2.403 \mathrm{E}+02$ \\
\hline WOA & $2.365 \mathrm{E}+04$ & $1.659 \mathrm{E}+04$ & $8.344 \mathrm{E}+05$ & $5.160 \mathrm{E}+05$ & $2.945 \mathrm{E}+03$ & $2.306 \mathrm{E}+02$ \\
\hline GWO & $1.684 \mathrm{E}+04$ & $7.003 \mathrm{E}+03$ & $3.185 \mathrm{E}+05$ & $3.125 \mathrm{E}+05$ & $2.521 \mathrm{E}+03$ & $1.812 \mathrm{E}+02$ \\
\hline GSA & $3.018 \mathrm{E}+04$ & $1.027 \mathrm{E}+04$ & $9.441 \mathrm{E}+04$ & $5.127 \mathrm{E}+04$ & $3.106 \mathrm{E}+03$ & $1.850 \mathrm{E}+02$ \\
\hline \multirow{2}{*}{ Algorithm } & \multicolumn{2}{|c|}{ F46 } & \multicolumn{2}{|c|}{ F47 } & \multicolumn{2}{|c|}{ F48 } \\
\hline & AVG & STD & AVG & STD & AVG & STD \\
\hline CPA & $2.500 \mathrm{E}+03$ & $0.000 \mathrm{E}+00$ & $2.600 \mathrm{E}+03$ & $0.000 \mathrm{E}+00$ & $2.700 \mathrm{E}+03$ & $0.000 \mathrm{E}+00$ \\
\hline PSO & $2.616 \mathrm{E}+03$ & $4.963 \mathrm{E}-01$ & $2.625 \mathrm{E}+03$ & $5.875 \mathrm{E}+00$ & $2.713 \mathrm{E}+03$ & $5.109 \mathrm{E}+00$ \\
\hline $\mathrm{DE}$ & $2.615 \mathrm{E}+03$ & $1.388 \mathrm{E}-12$ & $2.626 \mathrm{E}+03$ & $3.348 \mathrm{E}+00$ & $2.706 \mathrm{E}+03$ & $8.967 \mathrm{E}-01$ \\
\hline $\mathrm{ABC}$ & $2.615 \mathrm{E}+03$ & $1.412 \mathrm{E}-01$ & $2.625 \mathrm{E}+03$ & $4.099 \mathrm{E}+00$ & $2.707 \mathrm{E}+03$ & $1.074 \mathrm{E}+00$ \\
\hline SCA & $2.662 \mathrm{E}+03$ & $1.186 \mathrm{E}+01$ & $2.600 \mathrm{E}+03$ & $3.539 \mathrm{E}-02$ & $2.723 \mathrm{E}+03$ & $7.575 \mathrm{E}+00$ \\
\hline SSA & $2.615 \mathrm{E}+03$ & $2.895 \mathrm{E}-03$ & $2.636 \mathrm{E}+03$ & $6.940 \mathrm{E}+00$ & $2.710 \mathrm{E}+03$ & $3.060 \mathrm{E}+00$ \\
\hline BA & $2.615 \mathrm{E}+03$ & $1.040 \mathrm{E}-03$ & $2.672 \mathrm{E}+03$ & $3.814 \mathrm{E}+01$ & $2.731 \mathrm{E}+03$ & $9.406 \mathrm{E}+00$ \\
\hline FA & $2.725 \mathrm{E}+03$ & $1.847 \mathrm{E}+01$ & $2.704 \mathrm{E}+03$ & $4.337 \mathrm{E}+00$ & $2.733 \mathrm{E}+03$ & $4.208 \mathrm{E}+00$ \\
\hline MFO & $2.687 \mathrm{E}+03$ & $5.763 \mathrm{E}+01$ & $2.673 \mathrm{E}+03$ & $3.493 \mathrm{E}+01$ & $2.721 \mathrm{E}+03$ & $1.060 \mathrm{E}+01$ \\
\hline WOA & $2.620 \mathrm{E}+03$ & $3.321 \mathrm{E}+01$ & $2.610 \mathrm{E}+03$ & $2.405 \mathrm{E}+01$ & $2.717 \mathrm{E}+03$ & $1.470 \mathrm{E}+01$ \\
\hline GWO & $2.633 \mathrm{E}+03$ & $8.369 \mathrm{E}+00$ & $2.600 \mathrm{E}+03$ & $5.069 \mathrm{E}-04$ & $2.710 \mathrm{E}+03$ & $4.898 \mathrm{E}+00$ \\
\hline GSA & $2.615 \mathrm{E}+03$ & $5.088 \mathrm{E}+00$ & $2.608 \mathrm{E}+03$ & $4.604 \mathrm{E}-01$ & $2.702 \mathrm{E}+03$ & $1.348 \mathrm{E}-01$ \\
\hline \multirow{2}{*}{ Algorithm } & \multicolumn{2}{|c|}{ F49 } & \multicolumn{2}{|c|}{ F50 } & \multicolumn{2}{|c|}{ F51 } \\
\hline & AVG & STD & AVG & STD & AVG & STD \\
\hline CPA & $2.701 \mathrm{E}+03$ & $1.453 \mathrm{E}-01$ & $2.900 \mathrm{E}+03$ & $0.000 \mathrm{E}+00$ & $3.000 \mathrm{E}+03$ & $0.000 \mathrm{E}+00$ \\
\hline PSO & $2.790 \mathrm{E}+03$ & $3.055 \mathrm{E}+01$ & $3.468 \mathrm{E}+03$ & $2.768 \mathrm{E}+02$ & $7.227 \mathrm{E}+03$ & $9.782 \mathrm{E}+02$ \\
\hline $\mathrm{DE}$ & $2.700 \mathrm{E}+03$ & $3.038 \mathrm{E}-02$ & $3.182 \mathrm{E}+03$ & $8.413 \mathrm{E}+01$ & $3.630 \mathrm{E}+03$ & $1.937 \mathrm{E}+01$ \\
\hline $\mathrm{ABC}$ & $2.700 \mathrm{E}+03$ & $6.648 \mathrm{E}-02$ & $3.106 \mathrm{E}+03$ & $2.243 \mathrm{E}+00$ & $3.762 \mathrm{E}+03$ & $6.203 \mathrm{E}+01$ \\
\hline SCA & $2.702 \mathrm{E}+03$ & $4.861 \mathrm{E}-01$ & $3.360 \mathrm{E}+03$ & $2.629 \mathrm{E}+02$ & $4.714 \mathrm{E}+03$ & $2.778 \mathrm{E}+02$ \\
\hline SSA & $2.700 \mathrm{E}+03$ & $1.015 \mathrm{E}-01$ & $3.351 \mathrm{E}+03$ & $1.508 \mathrm{E}+02$ & $3.840 \mathrm{E}+03$ & $1.753 \mathrm{E}+02$ \\
\hline BA & $2.701 \mathrm{E}+03$ & $1.337 \mathrm{E}-01$ & $3.874 \mathrm{E}+03$ & $4.408 \mathrm{E}+02$ & $5.300 \mathrm{E}+03$ & $6.919 \mathrm{E}+02$ \\
\hline FA & $2.702 \mathrm{E}+03$ & $3.247 \mathrm{E}-01$ & $3.787 \mathrm{E}+03$ & $4.912 \mathrm{E}+01$ & $4.227 \mathrm{E}+03$ & $1.915 \mathrm{E}+02$ \\
\hline MFO & $2.706 \mathrm{E}+03$ & $1.835 \mathrm{E}+01$ & $3.615 \mathrm{E}+03$ & $2.342 \mathrm{E}+02$ & $3.966 \mathrm{E}+03$ & $1.962 \mathrm{E}+02$ \\
\hline WOA & $2.704 \mathrm{E}+03$ & $1.818 \mathrm{E}+01$ & $3.694 \mathrm{E}+03$ & $3.855 \mathrm{E}+02$ & $4.874 \mathrm{E}+03$ & $6.993 \mathrm{E}+02$ \\
\hline GWO & $2.741 \mathrm{E}+03$ & $5.901 \mathrm{E}+01$ & $3.329 \mathrm{E}+03$ & $1.234 \mathrm{E}+02$ & $3.940 \mathrm{E}+03$ & $2.723 \mathrm{E}+02$ \\
\hline GSA & $2.771 \mathrm{E}+03$ & $4.507 \mathrm{E}+01$ & $3.214 \mathrm{E}+03$ & $1.472 \mathrm{E}+02$ & $4.571 \mathrm{E}+03$ & $3.781 \mathrm{E}+02$ \\
\hline
\end{tabular}




\begin{tabular}{|c|c|c|c|c|c|c|c|}
\hline \multirow{2}{*}{ Algorithm } & \multicolumn{2}{|c|}{ F52 } & \multicolumn{2}{|c|}{ F53 } & \multirow[b]{2}{*}{$+1-1=$} & \multirow[b]{2}{*}{ Avg } & \multirow[b]{2}{*}{ Rank } \\
\hline & AVG & STD & AVG & STD & & & \\
\hline CPA & $3.100 E+03$ & $0.000 E+00$ & $3.200 \mathrm{E}+03$ & $0.000 E+00$ & $\sim$ & 3.20 & 1 \\
\hline PSO & $6.624 \mathrm{E}+04$ & $1.261 \mathrm{E}+05$ & $1.115 \mathrm{E}+04$ & $4.527 \mathrm{E}+03$ & $48 / 2 / 3$ & 9.21 & 10 \\
\hline $\mathrm{DE}$ & $8.522 \mathrm{E}+03$ & $2.019 \mathrm{E}+04$ & $5.963 \mathrm{E}+03$ & $9.032 \mathrm{E}+02$ & $27 / 14 / 12$ & 4.13 & 2 \\
\hline $\mathrm{ABC}$ & $3.851 \mathrm{E}+03$ & $8.538 \mathrm{E}+01$ & $5.145 \mathrm{E}+03$ & $3.938 \mathrm{E}+02$ & $26 / 20 /$ & 4.34 & 3 \\
\hline SCA & $9.763 \mathrm{E}+06$ & $6.364 \mathrm{E}+06$ & $2.048 \mathrm{E}+05$ & $6.666 \mathrm{E}+04$ & $51 / 0 / 2$ & 9.85 & 11 \\
\hline SSA & $1.922 \mathrm{E}+06$ & $4.382 \mathrm{E}+06$ & $9.973 \mathrm{E}+03$ & $2.606 \mathrm{E}+03$ & $35 / 3 / 1$ & 5.80 & 4 \\
\hline BA & $3.087 \mathrm{E}+07$ & $3.391 \mathrm{E}+07$ & $1.264 \mathrm{E}+04$ & $8.667 \mathrm{E}+03$ & $44 / 2 / 7$ & 8.76 & 9 \\
\hline FA & $2.610 \mathrm{E}+06$ & $1.028 \mathrm{E}+06$ & $1.618 \mathrm{E}+05$ & $4.247 \mathrm{E}+04$ & $50 / 0 / 3$ & 11.35 & 12 \\
\hline MFO & $3.518 \mathrm{E}+06$ & $4.109 \mathrm{E}+06$ & $5.656 \mathrm{E}+04$ & $5.039 \mathrm{E}+04$ & $46 / 0 / 7$ & 8.51 & 8 \\
\hline WOA & $5.810 \mathrm{E}+06$ & $4.500 \mathrm{E}+06$ & $7.142 \mathrm{E}+04$ & $4.129 \mathrm{E}+04$ & $44 / 1 / 8$ & 7.21 & 6 \\
\hline GWO & $3.878 \mathrm{E}+05$ & $9.711 \mathrm{E}+05$ & $5.055 \mathrm{E}+04$ & $4.784 \mathrm{E}+04$ & $41 / 4 / 8$ & 6.75 & 5 \\
\hline GSA & $4.758 \mathrm{E}+07$ & $5.944 \mathrm{E}+07$ & $7.193 \mathrm{E}+03$ & $8.104 \mathrm{E}+02$ & $45 / 5 / 3$ & 8.13 & 7 \\
\hline
\end{tabular}

F53 belong to CEC2014 functions. Of these, F26 is unimodal; F28, F35, and F39 are simple multimodal; F43 is a hybrid; and F50, F52, and F53 are composition. Fig. 7 shows CPA found the optimal solution on F1 and F4 early in the evaluation when other algorithms were just beginning to converge, suggesting that CPA is faster and more accurate than its competitors. Although CPA fell into local optimum on F26, it still ranked as the second-best algorithm. It can be observed from F10 and F20 that CPA found the optimal solution at a very fast speed in the early evaluation period, while some of the comparative algorithms fell into the local optimum. For F28, F39, and F43, CPA was still the first algorithm to find the optimal solution, although its convergence rate was slower. We can infer from the results that CPA's exploring and exploiting abilities are powerful, and the two stages have a good balance.

\subsection{Parameter sensitivity analysis}

Since an algorithm's parameters affect its convergence speed and accuracy, we analyzed the following parameters of CPA: Population size $(N)$, the maximum number of evaluations (MaxFEs), parameter ( $w$ ) and abandonment of the upper limit of probability. We set the abandonment probability upper limit to start from $a$ when the abandonment probability upper limit was tested. The parameter of $a$ was fixed to $w=9$ for a total of eleven values. Similarly, in the analysis of $a$, we initialized the upper limit of probability to $2 / 3 a$ and $w$ to 1 , 2, 3, 4, 5, 6, 7, 8, 9, 10, respectively. $N$ and MaxFEs were set to 30 and 1000, respectively, and remained unchanged when the test abandoned the upper limit of probability. Each algorithm was tested on the 53 functions 30 times.

Table 3 provides a reference for comparing different values of the abandonment probability upper limit, which has a significant impact on an algorithm's performance. Specifically, the best performance was achieved when the abandonment of the probability upper limit was $2 / 3 a$. Moreover, the maximum difference between the averages reached 3.7. Table 4 shows the influence of coefficient $w$ is relatively small, and the maximum difference between the average is only about 1.2 .

We used benchmark function F7 to test the influence of $N$ and MaxFEs on CPA, where $N$ was set to 5, 10, 30,50 , and 100, respectively. MaxFEs was initialized to $50,100,200,500$, and 1000. According to the test results in Fig. 8, the increase of $N$ and MaxFEs improved the accuracy of CPA, but when they reached a certain level, the influence becomes minimal. Therefore, it is suggested to set these values according to specific needs in an experiment, because if the values are too large or too small, convergence will take a long time and results will not be ideal.

\section{Comparative performance of CPA on en- gineering design problems}

To further evaluate its efficiency, CPA was tested on various engineering design problems. The constraints included the death penalty and annealing, static, 

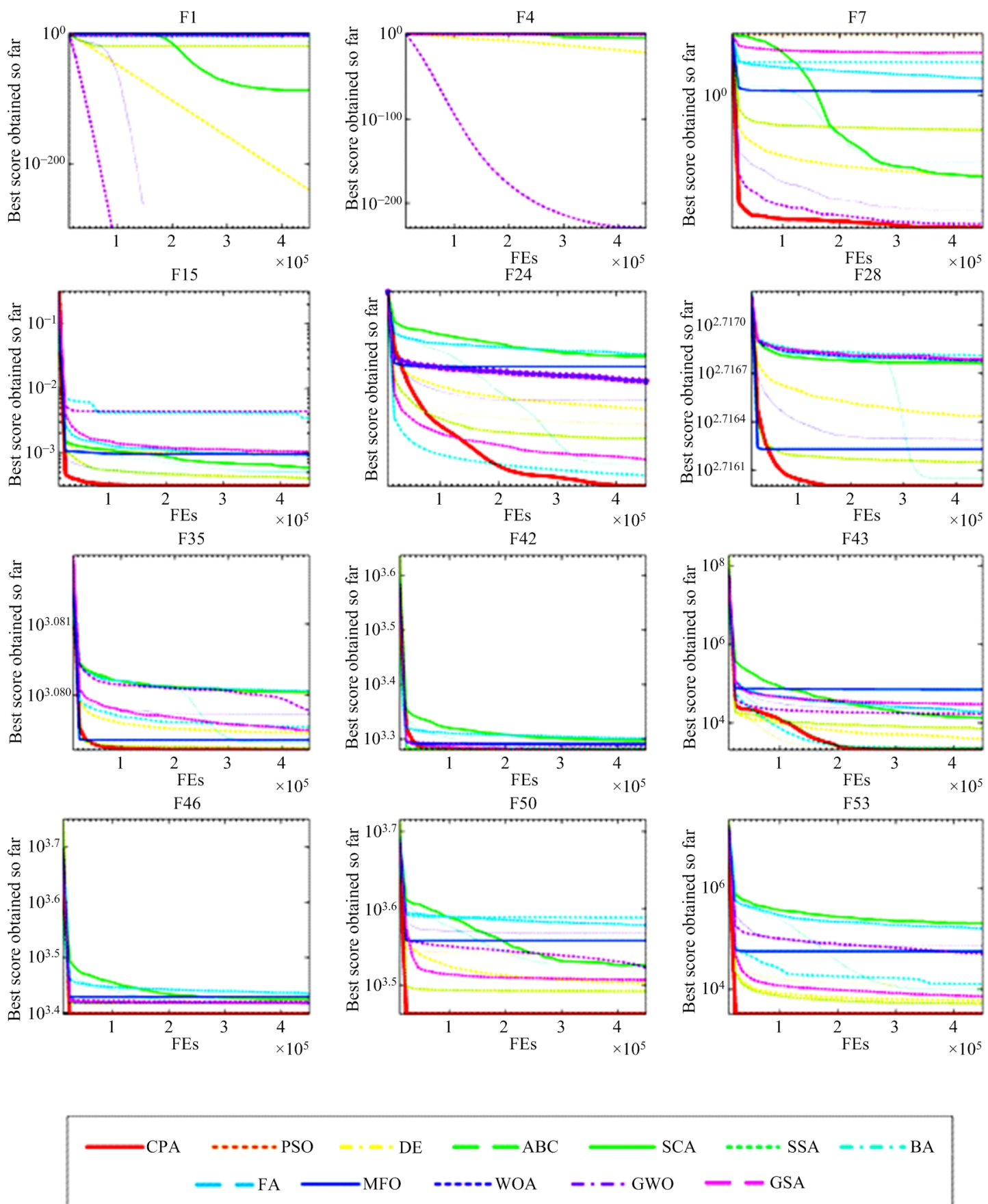

Fig. 6 Comparisons between CPA and traditional MAs.

dynamic, co-evolutionary, and adaptive behavior. To narrow down results, we selected the death penalty for comparisons in this work. The method assigns a large objective function value to them when searching individuals that violate any constraints. This method aims to automatically eliminate infeasible solutions in the heuristic algorithm's optimization, so it is unnecessary to calculate this scheme's infeasibility. The most prominent advantages of the death penalty are simplicity and low time consumption.

CPA was tested on the following five engineering constraints: Tension-compression spring, Welded beam, Pressure vessel, I-beam, Multiple disk clutch brake. 
Table 2 Comparison results of CPA with the advanced algorithms on the 53 functions

\begin{tabular}{|c|c|c|c|c|c|c|}
\hline \multirow{2}{*}{ Algorithm } & \multicolumn{2}{|c|}{$\mathrm{F} 1$} & \multicolumn{2}{|c|}{$\mathrm{F} 2$} & \multicolumn{2}{|c|}{ F3 } \\
\hline & AVG & STD & AVG & STD & AVG & STD \\
\hline CPA & $0.000 \mathrm{E}+00$ & $0.000 E+00$ & $0.000 E+00$ & $0.000 E+00$ & $0.000 \mathrm{E}+00$ & $0.000 E+00$ \\
\hline m_SCA & $0.000 E+00$ & $0.000 E+00$ & $0.000 E+00$ & $0.000 E+00$ & $2.69 \mathrm{E}-282$ & $0.000 \mathrm{E}+00$ \\
\hline OBSCA & $1.25 \mathrm{E}-158$ & $6.74 \mathrm{E}-158$ & $1.831 \mathrm{E}-137$ & $5.538 \mathrm{E}-137$ & $5.076 \mathrm{E}-38$ & $2.780 \mathrm{E}-37$ \\
\hline MWOA & $2.188 \mathrm{E}+04$ & $7.718 \mathrm{E}+03$ & $2.974 \mathrm{E}+05$ & $1.042 \mathrm{E}+06$ & $8.510 \mathrm{E}+04$ & $3.019 \mathrm{E}+04$ \\
\hline IWOA & $0.000 E+00$ & $0.000 E+00$ & $0.000 \mathrm{E}+00$ & $0.000 \mathrm{E}+00$ & $5.047 \mathrm{E}+00$ & $1.128 \mathrm{E}+01$ \\
\hline CDLOBA & $1.048 \mathrm{E}-04$ & $3.073 \mathrm{E}-05$ & $1.992 \mathrm{E}+01$ & $8.798 \mathrm{E}+01$ & $4.639 \mathrm{E}-04$ & $1.845 \mathrm{E}-04$ \\
\hline CBA & $1.415 \mathrm{E}-08$ & $6.143 \mathrm{E}-08$ & $1.357 \mathrm{E}+03$ & $7.331 \mathrm{E}+03$ & $1.052 \mathrm{E}+01$ & $5.026 \mathrm{E}+00$ \\
\hline CGPSO & $1.492 \mathrm{E}-09$ & $2.176 \mathrm{E}-09$ & $1.968 \mathrm{E}-05$ & $1.915 \mathrm{E}-05$ & $4.940 \mathrm{E}-02$ & $4.483 \mathrm{E}-02$ \\
\hline ALCPSO & $2.514 \mathrm{E}-188$ & $0.000 \mathrm{E}+00$ & $1.377 \mathrm{E}-05$ & $7.325 \mathrm{E}-05$ & $1.946 \mathrm{E}-18$ & $3.703 \mathrm{E}-18$ \\
\hline DECLS & $8.79 \mathrm{E}-119$ & $1.32 \mathrm{E}-118$ & $2.079 \mathrm{E}-70$ & $1.745 \mathrm{E}-70$ & $1.583 \mathrm{E}-05$ & $7.473 \mathrm{E}-05$ \\
\hline \multirow{2}{*}{ Algorithm } & \multicolumn{2}{|l|}{$\mathrm{F} 4$} & \multicolumn{2}{|l|}{ F5 } & \multicolumn{2}{|l|}{ F6 } \\
\hline & AVG & STD & AVG & STD & AVG & STD \\
\hline CPA & $0.000 \mathrm{E}+00$ & $0.000 E+00$ & $2.145 \mathrm{E}+01$ & $1.831 \mathrm{E}-01$ & $1.551 \mathrm{E}-26$ & $2.841 \mathrm{E}-26$ \\
\hline m_SCA & $5.14 \mathrm{E}-221$ & $0.000 E+00$ & $2.679 \mathrm{E}+01$ & $1.063 \mathrm{E}+00$ & $2.344 \mathrm{E}+00$ & $4.193 \mathrm{E}-01$ \\
\hline OBSCA & $1.060 \mathrm{E}-34$ & $4.974 \mathrm{E}-34$ & $2.771 \mathrm{E}+01$ & $5.362 \mathrm{E}-01$ & $3.844 \mathrm{E}+00$ & $1.717 \mathrm{E}-01$ \\
\hline MWOA & $6.955 \mathrm{E}-01$ & $2.374 \mathrm{E}+00$ & $3.659 \mathrm{E}+07$ & $1.940 \mathrm{E}+07$ & $2.327 \mathrm{E}+04$ & $7.569 \mathrm{E}+03$ \\
\hline IWOA & $1.010 \mathrm{E}-09$ & $5.531 \mathrm{E}-09$ & $2.279 \mathrm{E}+01$ & $6.380 \mathrm{E}-01$ & $8.517 \mathrm{E}-07$ & $3.519 \mathrm{E}-07$ \\
\hline CDLOBA & $4.051 \mathrm{E}+01$ & $7.625 \mathrm{E}+00$ & $5.148 \mathrm{E}+01$ & $7.075 \mathrm{E}+01$ & $1.001 \mathrm{E}-04$ & $3.649 \mathrm{E}-05$ \\
\hline CBA & $6.342 \mathrm{E}+00$ & $7.897 \mathrm{E}+00$ & $8.785 \mathrm{E}+01$ & $1.163 \mathrm{E}+02$ & $2.454 \mathrm{E}-08$ & $1.232 \mathrm{E}-07$ \\
\hline CGPSO & $4.628 \mathrm{E}-06$ & $4.775 \mathrm{E}-06$ & $1.701 E-08$ & $4.212 \mathrm{E}-08$ & $1.360 \mathrm{E}-09$ & $1.673 \mathrm{E}-09$ \\
\hline ALCPSO & $3.215 \mathrm{E}-08$ & $2.936 \mathrm{E}-08$ & $3.205 \mathrm{E}+01$ & $3.083 \mathrm{E}+01$ & $3.255 \mathrm{E}-30$ & $1.661 \mathrm{E}-29$ \\
\hline DECLS & $3.212 \mathrm{E}-11$ & $1.773 \mathrm{E}-11$ & $7.748 \mathrm{E}-09$ & $1.262 \mathrm{E}-08$ & $0.000 \mathrm{E}+00$ & $0.000 \mathrm{E}+00$ \\
\hline \multirow{2}{*}{ Algorithm } & \multicolumn{2}{|c|}{ F7 } & \multicolumn{2}{|c|}{ F8 } & \multicolumn{2}{|c|}{ F9 } \\
\hline & AVG & STD & AVG & STD & AVG & STD \\
\hline CPA & $7.115 \mathrm{E}-05$ & $1.705 \mathrm{E}-04$ & $-1.257 \mathrm{E}+04$ & $5.189 \mathrm{E}-12$ & $0.000 E+00$ & $0.000 E+00$ \\
\hline m_SCA & $4.159 \mathrm{E}-05$ & $2.817 \mathrm{E}-05$ & $-7.045 \mathrm{E}+03$ & $6.606 \mathrm{E}+02$ & $0.000 \mathrm{E}+00$ & $0.000 \mathrm{E}+00$ \\
\hline OBSCA & $6.913 \mathrm{E}-04$ & $3.587 \mathrm{E}-04$ & $-4.200 \mathrm{E}+03$ & $2.450 \mathrm{E}+02$ & $0.000 \mathrm{E}+00$ & $0.000 E+00$ \\
\hline MWOA & $2.549 \mathrm{E}+01$ & $2.048 \mathrm{E}+01$ & $-5.421 \mathrm{E}+03$ & $6.948 \mathrm{E}+02$ & $3.047 \mathrm{E}+02$ & $4.168 \mathrm{E}+01$ \\
\hline IWOA & $1.077 \mathrm{E}-04$ & $1.085 \mathrm{E}-04$ & $-1.221 \mathrm{E}+04$ & $9.975 \mathrm{E}+02$ & $0.000 E+00$ & $0.000 E+00$ \\
\hline CDLOBA & $1.065 \mathrm{E}-02$ & $1.053 \mathrm{E}-02$ & $-7.179 \mathrm{E}+03$ & $6.898 \mathrm{E}+02$ & $1.298 \mathrm{E}+02$ & $5.966 \mathrm{E}+01$ \\
\hline CBA & $1.206 \mathrm{E}-01$ & $3.890 \mathrm{E}-01$ & $-7.141 \mathrm{E}+03$ & $7.509 \mathrm{E}+02$ & $1.214 \mathrm{E}+02$ & $4.671 \mathrm{E}+01$ \\
\hline CGPSO & $7.411 \mathrm{E}-06$ & $5.534 \mathrm{E}-06$ & $-4.103 E+04$ & $8.845 \mathrm{E}+03$ & $2.141 \mathrm{E}-09$ & $3.239 \mathrm{E}-09$ \\
\hline ALCPSO & $7.510 \mathrm{E}-02$ & $2.667 \mathrm{E}-02$ & $-1.172 \mathrm{E}+04$ & $2.705 \mathrm{E}+02$ & $1.636 \mathrm{E}+01$ & $8.408 \mathrm{E}+00$ \\
\hline DECLS & $9.693 \mathrm{E}-06$ & $1.044 \mathrm{E}-05$ & $-1.257 \mathrm{E}+04$ & $2.464 \mathrm{E}-09$ & $1.660 \mathrm{E}-11$ & $8.776 \mathrm{E}-11$ \\
\hline \multirow{2}{*}{ Algorithm } & \multicolumn{2}{|c|}{$\mathrm{F} 10$} & \multicolumn{2}{|c|}{ F11 } & \multicolumn{2}{|c|}{ F12 } \\
\hline & $\mathrm{AVG}$ & STD & AVG & STD & AVG & STD \\
\hline CPA & $8.882 E-16$ & $0.000 E+00$ & $0.000 \mathrm{E}+00$ & $0.000 \mathrm{E}+00$ & $3.749 \mathrm{E}-29$ & $1.276 \mathrm{E}-28$ \\
\hline m_SCA & $6.672 \mathrm{E}-01$ & $3.655 \mathrm{E}+00$ & $0.000 E+00$ & $0.000 \mathrm{E}+00$ & $1.415 \mathrm{E}-01$ & $4.864 \mathrm{E}-02$ \\
\hline OBSCA & $4.441 \mathrm{E}-15$ & $0.000 E+00$ & $0.000 E+00$ & $0.000 \mathrm{E}+00$ & $3.913 \mathrm{E}-01$ & $8.537 \mathrm{E}-02$ \\
\hline MWOA & $1.813 \mathrm{E}+01$ & $1.282 \mathrm{E}+00$ & $2.314 \mathrm{E}+02$ & $5.463 \mathrm{E}+01$ & $6.103 \mathrm{E}+07$ & $6.883 \mathrm{E}+07$ \\
\hline IWOA & $2.783 \mathrm{E}-15$ & $1.803 \mathrm{E}-15$ & $0.000 \mathrm{E}+00$ & $0.000 \mathrm{E}+00$ & $1.396 \mathrm{E}-07$ & $5.572 \mathrm{E}-08$ \\
\hline CDLOBA & $1.935 \mathrm{E}+01$ & $9.355 \mathrm{E}-01$ & $6.647 \mathrm{E}+01$ & $6.601 \mathrm{E}+01$ & $1.695 \mathrm{E}+01$ & $8.470 \mathrm{E}+00$ \\
\hline CBA & $1.538 \mathrm{E}+01$ & $3.469 \mathrm{E}+00$ & $1.289 \mathrm{E}-02$ & $1.177 \mathrm{E}-02$ & $1.644 \mathrm{E}+01$ & $5.412 \mathrm{E}+00$ \\
\hline CGPSO & $1.107 \mathrm{E}-05$ & $1.342 \mathrm{E}-05$ & $4.741 \mathrm{E}-09$ & $8.832 \mathrm{E}-09$ & $2.198 \mathrm{E}-11$ & $2.468 \mathrm{E}-11$ \\
\hline
\end{tabular}


Journal of Bionic Engineering (2021) Vol.18 No.3

\begin{tabular}{|c|c|c|c|c|c|c|}
\hline ALCPSO & $1.135 \mathrm{E}+00$ & $8.861 \mathrm{E}-01$ & $2.602 \mathrm{E}-02$ & $2.699 \mathrm{E}-02$ & $1.227 \mathrm{E}-02$ & $3.621 \mathrm{E}-02$ \\
\hline DECLS & $7.875 \mathrm{E}-15$ & $6.486 \mathrm{E}-16$ & $0.000 E+00$ & $0.000 \mathrm{E}+00$ & $1.571 E-32$ & $5.567 \mathrm{E}-48$ \\
\hline \multirow{2}{*}{ Algorithm } & \multicolumn{2}{|c|}{ F13 } & \multicolumn{2}{|c|}{$\mathrm{F} 14$} & \multicolumn{2}{|c|}{ F15 } \\
\hline & AVG & STD & AVG & STD & AVG & STD \\
\hline CPA & $4.554 \mathrm{E}-28$ & $7.038 \mathrm{E}-28$ & $9.980 \mathrm{E}-01$ & $1.487 \mathrm{E}-16$ & $3.075 E-04$ & $5.112 \mathrm{E}-17$ \\
\hline m_SCA & $1.503 \mathrm{E}+00$ & $2.505 \mathrm{E}-01$ & $1.130 \mathrm{E}+00$ & $5.034 \mathrm{E}-01$ & $4.296 \mathrm{E}-04$ & $3.166 \mathrm{E}-04$ \\
\hline OBSCA & $2.125 \mathrm{E}+00$ & $1.176 \mathrm{E}-01$ & $1.196 \mathrm{E}+00$ & $6.054 \mathrm{E}-01$ & $4.695 \mathrm{E}-04$ & $1.609 \mathrm{E}-04$ \\
\hline MWOA & $1.415 \mathrm{E}+08$ & $1.735 \mathrm{E}+08$ & $5.326 \mathrm{E}+00$ & $4.986 \mathrm{E}+00$ & $2.792 \mathrm{E}-02$ & $3.377 \mathrm{E}-02$ \\
\hline IWOA & $2.195 \mathrm{E}-06$ & $1.160 \mathrm{E}-06$ & $9.980 \mathrm{E}-01$ & $9.823 \mathrm{E}-16$ & $3.381 \mathrm{E}-04$ & $1.672 \mathrm{E}-04$ \\
\hline CDLOBA & $3.824 \mathrm{E}+01$ & $1.265 \mathrm{E}+01$ & $1.989 \mathrm{E}+00$ & $1.219 \mathrm{E}+00$ & $3.331 \mathrm{E}-03$ & $6.798 \mathrm{E}-03$ \\
\hline CBA & $2.863 \mathrm{E}+01$ & $2.863 \mathrm{E}+01$ & $1.856 \mathrm{E}+00$ & $1.342 \mathrm{E}+00$ & $7.041 \mathrm{E}-03$ & $1.506 \mathrm{E}-02$ \\
\hline CGPSO & $2.732 \mathrm{E}-10$ & $4.180 \mathrm{E}-10$ & $1.064 \mathrm{E}+00$ & $2.522 \mathrm{E}-01$ & $6.631 \mathrm{E}-04$ & $2.003 \mathrm{E}-04$ \\
\hline ALCPSO & $1.367 \mathrm{E}-02$ & $5.440 \mathrm{E}-02$ & $9.980 E-01$ & $9.220 \mathrm{E}-17$ & $3.380 \mathrm{E}-04$ & $1.672 \mathrm{E}-04$ \\
\hline DECLS & $1.350 \mathrm{E}-32$ & $5.567 \mathrm{E}-48$ & $1.034 \mathrm{E}+00$ & $1.983 \mathrm{E}-01$ & $4.445 \mathrm{E}-04$ & $3.313 \mathrm{E}-04$ \\
\hline \multirow{2}{*}{ Algorithm } & \multicolumn{2}{|c|}{ F16 } & \multicolumn{2}{|c|}{$\mathrm{F} 17$} & \multicolumn{2}{|c|}{ F18 } \\
\hline & AVG & STD & AVG & STD & AVG & STD \\
\hline CPA & $-1.032 \mathrm{E}+00$ & $5.684 \mathrm{E}-16$ & $3.979 \mathrm{E}-01$ & $0.000 \mathrm{E}+00$ & $3.000 \mathrm{E}+00$ & $7.823 E-16$ \\
\hline m_SCA & $-1.032 E+00$ & $2.585 \mathrm{E}-10$ & $3.979 \mathrm{E}-01$ & $3.744 \mathrm{E}-09$ & $3.000 \mathrm{E}+00$ & $5.593 \mathrm{E}-08$ \\
\hline OBSCA & $-1.032 E+00$ & $2.722 \mathrm{E}-08$ & $3.980 \mathrm{E}-01$ & $6.649 \mathrm{E}-05$ & $3.000 \mathrm{E}+00$ & $6.572 \mathrm{E}-07$ \\
\hline MWOA & $-9.257 \mathrm{E}-01$ & $1.847 \mathrm{E}-01$ & $5.182 \mathrm{E}-01$ & $2.130 \mathrm{E}-01$ & $8.746 \mathrm{E}+00$ & $1.076 \mathrm{E}+01$ \\
\hline IWOA & $-1.032 E+00$ & $9.749 \mathrm{E}-16$ & $3.979 \mathrm{E}-01$ & $1.018 \mathrm{E}-11$ & $3.000 \mathrm{E}+00$ & $3.780 \mathrm{E}-10$ \\
\hline CDLOBA & $-1.032 E+00$ & $1.318 \mathrm{E}-05$ & $3.979 \mathrm{E}-01$ & $8.478 \mathrm{E}-06$ & $3.001 \mathrm{E}+00$ & $1.366 \mathrm{E}-03$ \\
\hline $\mathrm{CBA}$ & $-1.032 E+00$ & $1.485 \mathrm{E}-06$ & $3.979 \mathrm{E}-01$ & $6.915 \mathrm{E}-07$ & $3.000 \mathrm{E}+00$ & $4.852 \mathrm{E}-05$ \\
\hline CGPSO & $-1.031 E+00$ & $1.913 \mathrm{E}-04$ & $3.980 \mathrm{E}-01$ & $1.001 \mathrm{E}-04$ & $3.010 \mathrm{E}+00$ & $1.180 \mathrm{E}-02$ \\
\hline ALCPSO & $-1.032 E+00$ & $5.904 \mathrm{E}-16$ & $3.979 \mathrm{E}-01$ & $0.000 E+00$ & $3.000 E+00$ & $2.109 \mathrm{E}-15$ \\
\hline DECLS & $-1.032 E+00$ & $6.775 \mathrm{E}-16$ & $3.979 \mathrm{E}-01$ & $0.000 E+00$ & $3.000 \mathrm{E}+00$ & $1.195 \mathrm{E}-15$ \\
\hline \multirow{2}{*}{ Algorithm } & \multicolumn{2}{|c|}{ F19 } & \multicolumn{2}{|c|}{$\mathrm{F} 20$} & \multicolumn{2}{|c|}{$\mathrm{F} 21$} \\
\hline & $\mathrm{AVG}$ & STD & AVG & STD & AVG & STD \\
\hline CPA & $-3.863 E+00$ & $2.542 \mathrm{E}-15$ & $-3.318 \mathrm{E}+00$ & $2.171 \mathrm{E}-02$ & $-7.774 \mathrm{E}+00$ & $2.587 \mathrm{E}+00$ \\
\hline m_SCA & $-3.863 E+00$ & $1.832 \mathrm{E}-06$ & $-3.240 \mathrm{E}+00$ & $5.923 \mathrm{E}-02$ & $-9.985 \mathrm{E}+00$ & $9.224 \mathrm{E}-01$ \\
\hline OBSCA & $-3.862 \mathrm{E}+00$ & $3.308 \mathrm{E}-04$ & $-3.280 \mathrm{E}+00$ & $1.890 \mathrm{E}-02$ & $-9.869 \mathrm{E}+00$ & $1.590 \mathrm{E}-01$ \\
\hline MWOA & $-3.735 \mathrm{E}+00$ & $1.091 \mathrm{E}-01$ & $-2.653 \mathrm{E}+00$ & $4.343 \mathrm{E}-01$ & $-3.343 \mathrm{E}+00$ & $1.847 \mathrm{E}+00$ \\
\hline IWOA & $-3.862 \mathrm{E}+00$ & $2.725 \mathrm{E}-03$ & $-3.242 \mathrm{E}+00$ & $1.005 \mathrm{E}-01$ & $-1.015 E+01$ & $1.058 \mathrm{E}-07$ \\
\hline CDLOBA & $-3.859 \mathrm{E}+00$ & $2.944 \mathrm{E}-03$ & $-3.129 \mathrm{E}+00$ & $4.376 \mathrm{E}-02$ & $-6.529 \mathrm{E}+00$ & $3.488 \mathrm{E}+00$ \\
\hline $\mathrm{CBA}$ & $-3.863 E+00$ & $5.284 \mathrm{E}-05$ & $-3.245 \mathrm{E}+00$ & $5.804 \mathrm{E}-02$ & $-5.054 \mathrm{E}+00$ & $3.042 \mathrm{E}+00$ \\
\hline CGPSO & $-3.860 \mathrm{E}+00$ & $2.761 \mathrm{E}-03$ & $-3.078 \mathrm{E}+00$ & $1.061 \mathrm{E}-01$ & $-1.015 E+01$ & $9.773 \mathrm{E}-11$ \\
\hline ALCPSO & $-3.863 E+00$ & $2.597 \mathrm{E}-15$ & $-3.286 \mathrm{E}+00$ & $5.542 \mathrm{E}-02$ & $-8.547 \mathrm{E}+00$ & $2.519 \mathrm{E}+00$ \\
\hline DECLS & $-3.863 E+00$ & $2.710 \mathrm{E}-15$ & $-3.322 \mathrm{E}+00$ & $3.626 \mathrm{E}-05$ & $-1.015 E+01$ & $7.227 \mathrm{E}-15$ \\
\hline \multirow{2}{*}{ Algorithm } & \multicolumn{2}{|c|}{ F22 } & \multicolumn{2}{|c|}{ F23 } & \multicolumn{2}{|c|}{ F24 } \\
\hline & AVG & STD & AVG & STD & Avg & Rank \\
\hline CPA & $-8.100 \mathrm{E}+00$ & $2.679 \mathrm{E}+00$ & $-8.734 \mathrm{E}+00$ & $2.593 \mathrm{E}+00$ & $3.290 \mathrm{E}+05$ & $1.350 \mathrm{E}+05$ \\
\hline m_SCA & $-9.876 \mathrm{E}+00$ & $1.609 \mathrm{E}+00$ & $-1.000 \mathrm{E}+01$ & $1.636 \mathrm{E}+00$ & $4.327 \mathrm{E}+07$ & $2.832 \mathrm{E}+07$ \\
\hline OBSCA & $-1.003 \mathrm{E}+01$ & $2.487 \mathrm{E}-01$ & $-1.011 \mathrm{E}+01$ & $2.475 \mathrm{E}-01$ & $3.882 \mathrm{E}+08$ & $1.112 \mathrm{E}+08$ \\
\hline MWOA & $-2.860 \mathrm{E}+00$ & $1.649 \mathrm{E}+00$ & $-3.080 \mathrm{E}+00$ & $1.724 \mathrm{E}+00$ & $1.857 \mathrm{E}+09$ & $6.892 \mathrm{E}+08$ \\
\hline IWOA & $-1.040 \mathrm{E}+01$ & $5.572 \mathrm{E}-08$ & $-1.054 \mathrm{E}+01$ & $5.190 \mathrm{E}-08$ & $1.393 \mathrm{E}+07$ & $8.157 \mathrm{E}+06$ \\
\hline CDLOBA & $-8.914 \mathrm{E}+00$ & $2.958 \mathrm{E}+00$ & $-8.115 \mathrm{E}+00$ & $3.453 \mathrm{E}+00$ & $3.238 E+05$ & $1.812 \mathrm{E}+05$ \\
\hline CBA & $-7.725 \mathrm{E}+00$ & $3.629 \mathrm{E}+00$ & $-6.424 \mathrm{E}+00$ & $3.940 \mathrm{E}+00$ & $3.991 \mathrm{E}+06$ & $1.312 \mathrm{E}+06$ \\
\hline CGPSO & $-1.040 E+01$ & $1.201 \mathrm{E}-10$ & $-1.054 \mathrm{E}+01$ & $7.860 \mathrm{E}-11$ & $8.169 \mathrm{E}+06$ & $2.222 \mathrm{E}+06$ \\
\hline ALCPSO & $-1.005 \mathrm{E}+01$ & $1.338 \mathrm{E}+00$ & $-1.032 \mathrm{E}+01$ & $9.933 \mathrm{E}-01$ & $6.319 \mathrm{E}+06$ & $5.585 \mathrm{E}+06$ \\
\hline
\end{tabular}


Tu et al.: The Colony Predation Algorithm

\begin{tabular}{|c|c|c|c|c|c|c|}
\hline DECLS & $-1.040 \mathrm{E}+01$ & $1.807 E-15$ & $-1.054 \mathrm{E}+01$ & $1.649 \mathrm{E}-15$ & $2.248 \mathrm{E}+07$ & $6.256 \mathrm{E}+06$ \\
\hline \multirow{2}{*}{ Algorithm } & \multicolumn{2}{|c|}{ F25 } & \multicolumn{2}{|c|}{ F26 } & \multicolumn{2}{|c|}{$\mathrm{F} 27$} \\
\hline & AVG & STD & AVG & STD & AVG & STD \\
\hline CPA & $1.766 \mathrm{E}+04$ & $1.141 \mathrm{E}+04$ & $3.565 \mathrm{E}+02$ & $3.827 E+01$ & $4.513 E+02$ & $4.223 \mathrm{E}+01$ \\
\hline m_SCA & $5.467 \mathrm{E}+09$ & $1.998 \mathrm{E}+09$ & $2.245 \mathrm{E}+04$ & $5.567 \mathrm{E}+03$ & $8.127 \mathrm{E}+02$ & $1.568 \mathrm{E}+02$ \\
\hline OBSCA & $2.280 \mathrm{E}+10$ & $3.356 \mathrm{E}+09$ & $4.783 \mathrm{E}+04$ & $7.849 \mathrm{E}+03$ & $2.223 \mathrm{E}+03$ & $7.348 \mathrm{E}+02$ \\
\hline MWOA & $9.901 \mathrm{E}+10$ & $2.208 \mathrm{E}+10$ & $5.752 \mathrm{E}+05$ & $5.535 \mathrm{E}+05$ & $2.441 \mathrm{E}+04$ & $1.004 \mathrm{E}+04$ \\
\hline IWOA & $4.516 \mathrm{E}+05$ & $4.627 \mathrm{E}+05$ & $1.306 \mathrm{E}+04$ & $7.367 \mathrm{E}+03$ & $5.586 \mathrm{E}+02$ & $5.647 \mathrm{E}+01$ \\
\hline CDLOBA & $9.936 \mathrm{E}+03$ & $1.068 \mathrm{E}+04$ & $6.104 \mathrm{E}+04$ & $2.018 \mathrm{E}+04$ & $4.883 \mathrm{E}+02$ & $5.822 \mathrm{E}+01$ \\
\hline CBA & $7.904 \mathrm{E}+04$ & $3.741 \mathrm{E}+05$ & $1.778 \mathrm{E}+03$ & $4.473 \mathrm{E}+03$ & $5.063 \mathrm{E}+02$ & $2.889 \mathrm{E}+01$ \\
\hline CGPSO & $1.460 \mathrm{E}+08$ & $2.219 \mathrm{E}+07$ & $1.234 \mathrm{E}+03$ & $1.957 \mathrm{E}+02$ & $4.746 \mathrm{E}+02$ & $4.260 \mathrm{E}+01$ \\
\hline ALCPSO & $1.912 \mathrm{E}+03$ & $2.375 \mathrm{E}+03$ & $3.779 \mathrm{E}+02$ & $2.252 \mathrm{E}+02$ & $5.273 \mathrm{E}+02$ & $3.530 \mathrm{E}+01$ \\
\hline DECLS & $7.740 \mathrm{E}+02$ & $1.881 \mathrm{E}+03$ & $5.267 \mathrm{E}+02$ & $4.877 \mathrm{E}+02$ & $4.984 \mathrm{E}+02$ & $2.097 \mathrm{E}+01$ \\
\hline \multirow{2}{*}{ Algorithm } & \multicolumn{2}{|c|}{ F28 } & \multicolumn{2}{|c|}{ F29 } & \multicolumn{2}{|c|}{ F30 } \\
\hline & AVG & STD & AVG & STD & AVG & STD \\
\hline CPA & $5.200 \mathrm{E}+02$ & $2.243 E-04$ & $6.160 \mathrm{E}+02$ & $2.534 \mathrm{E}+00$ & $7.000 \mathrm{E}+02$ & $2.455 \mathrm{E}-02$ \\
\hline m_SCA & $5.206 \mathrm{E}+02$ & $1.626 \mathrm{E}-01$ & $6.206 \mathrm{E}+02$ & $2.798 \mathrm{E}+00$ & $7.467 \mathrm{E}+02$ & $2.210 \mathrm{E}+01$ \\
\hline OBSCA & $5.209 \mathrm{E}+02$ & $4.723 \mathrm{E}-02$ & $6.316 \mathrm{E}+02$ & $1.393 E+00$ & $8.844 \mathrm{E}+02$ & $2.932 \mathrm{E}+01$ \\
\hline MWOA & $5.213 \mathrm{E}+02$ & $6.794 \mathrm{E}-02$ & $6.460 \mathrm{E}+02$ & $2.910 \mathrm{E}+00$ & $1.488 \mathrm{E}+03$ & $1.820 \mathrm{E}+02$ \\
\hline IWOA & $5.202 \mathrm{E}+02$ & $1.386 \mathrm{E}-01$ & $6.295 \mathrm{E}+02$ & $3.143 \mathrm{E}+00$ & $7.006 E+02$ & $1.653 \mathrm{E}-01$ \\
\hline CDLOBA & $5.207 \mathrm{E}+02$ & $3.399 \mathrm{E}-01$ & $6.361 \mathrm{E}+02$ & $3.370 \mathrm{E}+00$ & $7.000 \mathrm{E}+02$ & $9.591 \mathrm{E}-03$ \\
\hline $\mathrm{CBA}$ & $5.201 \mathrm{E}+02$ & $1.762 \mathrm{E}-01$ & $6.425 \mathrm{E}+02$ & $2.854 \mathrm{E}+00$ & $7.000 \mathrm{E}+02$ & $1.545 \mathrm{E}-02$ \\
\hline CGPSO & $5.210 \mathrm{E}+02$ & $5.258 \mathrm{E}-02$ & $6.228 \mathrm{E}+02$ & $3.066 \mathrm{E}+00$ & $7.023 \mathrm{E}+02$ & $1.555 \mathrm{E}-01$ \\
\hline ALCPSO & $5.208 \mathrm{E}+02$ & $6.867 \mathrm{E}-02$ & $6.164 \mathrm{E}+02$ & $4.277 \mathrm{E}+00$ & $7.000 \mathrm{E}+02$ & $1.289 \mathrm{E}-02$ \\
\hline DECLS & $5.206 \mathrm{E}+02$ & $4.869 \mathrm{E}-02$ & $6.203 E+02$ & $1.488 \mathrm{E}+00$ & $7.000 \mathrm{E}+02$ & $4.363 E-07$ \\
\hline \multirow{2}{*}{ Algorithm } & \multicolumn{2}{|c|}{ F31 } & \multicolumn{2}{|c|}{$\mathrm{F} 32$} & \multicolumn{2}{|c|}{ F33 } \\
\hline & AVG & STD & AVG & STD & AVG & STD \\
\hline CPA & $8.001 E+02$ & $2.524 \mathrm{E}-01$ & $1.017 \mathrm{E}+03$ & $2.203 \mathrm{E}+01$ & $1.002 E+03$ & $1.755 E+00$ \\
\hline m_SCA & $9.333 \mathrm{E}+02$ & $2.818 \mathrm{E}+01$ & $1.052 \mathrm{E}+03$ & $2.381 \mathrm{E}+01$ & $4.046 \mathrm{E}+03$ & $6.226 \mathrm{E}+02$ \\
\hline OBSCA & $1.051 \mathrm{E}+03$ & $1.785 \mathrm{E}+01$ & $1.192 \mathrm{E}+03$ & $1.630 \mathrm{E}+01$ & $6.039 \mathrm{E}+03$ & $3.769 \mathrm{E}+02$ \\
\hline MWOA & $1.239 \mathrm{E}+03$ & $5.465 \mathrm{E}+01$ & $1.383 \mathrm{E}+03$ & $6.136 \mathrm{E}+01$ & $9.545 \mathrm{E}+03$ & $5.128 \mathrm{E}+02$ \\
\hline IWOA & $9.066 \mathrm{E}+02$ & $1.714 \mathrm{E}+01$ & $1.114 \mathrm{E}+03$ & $5.163 \mathrm{E}+01$ & $2.445 \mathrm{E}+03$ & $3.908 \mathrm{E}+02$ \\
\hline CDLOBA & $1.049 \mathrm{E}+03$ & $3.861 \mathrm{E}+01$ & $1.229 \mathrm{E}+03$ & $6.754 \mathrm{E}+01$ & $5.347 \mathrm{E}+03$ & $6.767 \mathrm{E}+02$ \\
\hline $\mathrm{CBA}$ & $1.008 \mathrm{E}+03$ & $3.546 \mathrm{E}+01$ & $1.172 \mathrm{E}+03$ & $7.543 \mathrm{E}+01$ & $5.558 \mathrm{E}+03$ & $6.869 \mathrm{E}+02$ \\
\hline CGPSO & $9.805 \mathrm{E}+02$ & $2.737 \mathrm{E}+01$ & $1.119 \mathrm{E}+03$ & $2.733 \mathrm{E}+01$ & $5.669 \mathrm{E}+03$ & $5.223 \mathrm{E}+02$ \\
\hline ALCPSO & $8.197 \mathrm{E}+02$ & $1.079 \mathrm{E}+01$ & $9.980 \mathrm{E}+02$ & $2.808 \mathrm{E}+01$ & $1.412 \mathrm{E}+03$ & $2.965 \mathrm{E}+02$ \\
\hline DECLS & $8.011 \mathrm{E}+02$ & $1.051 \mathrm{E}+00$ & $1.015 \mathrm{E}+03$ & $8.373 E+00$ & $1.031 \mathrm{E}+03$ & $4.470 \mathrm{E}+01$ \\
\hline \multirow{2}{*}{ Algorithm } & \multicolumn{2}{|c|}{ F34 } & \multicolumn{2}{|c|}{ F35 } & \multicolumn{2}{|c|}{ F36 } \\
\hline & AVG & STD & AVG & STD & AVG & STD \\
\hline CPA & $3.579 \mathrm{E}+03$ & $4.997 \mathrm{E}+02$ & $1.200 \mathrm{E}+03$ & $4.286 \mathrm{E}-02$ & $1.301 \mathrm{E}+03$ & $1.009 \mathrm{E}-01$ \\
\hline m_SCA & $4.610 \mathrm{E}+03$ & $8.319 \mathrm{E}+02$ & $1.201 \mathrm{E}+03$ & $2.808 \mathrm{E}-01$ & $1.301 \mathrm{E}+03$ & $5.192 \mathrm{E}-01$ \\
\hline OBSCA & $7.075 \mathrm{E}+03$ & $4.943 \mathrm{E}+02$ & $1.202 \mathrm{E}+03$ & $2.713 \mathrm{E}-01$ & $1.303 \mathrm{E}+03$ & $3.772 \mathrm{E}-01$ \\
\hline MWOA & $1.007 \mathrm{E}+04$ & $4.826 \mathrm{E}+02$ & $1.206 \mathrm{E}+03$ & $9.982 \mathrm{E}-01$ & $1.309 \mathrm{E}+03$ & $1.134 \mathrm{E}+00$ \\
\hline IWOA & $5.427 \mathrm{E}+03$ & $7.208 \mathrm{E}+02$ & $1.201 \mathrm{E}+03$ & $2.658 \mathrm{E}-01$ & $1.301 \mathrm{E}+03$ & $1.045 \mathrm{E}-01$ \\
\hline CDLOBA & $5.652 \mathrm{E}+03$ & $7.017 \mathrm{E}+02$ & $1.200 \mathrm{E}+03$ & $2.214 \mathrm{E}-01$ & $1.300 \mathrm{E}+03$ & $1.134 \mathrm{E}-01$ \\
\hline CBA & $5.763 \mathrm{E}+03$ & $8.245 \mathrm{E}+02$ & $1.201 \mathrm{E}+03$ & $4.547 \mathrm{E}-01$ & $1.300 \mathrm{E}+03$ & $1.406 \mathrm{E}-01$ \\
\hline CGPSO & $6.061 \mathrm{E}+03$ & $4.943 \mathrm{E}+02$ & $1.202 \mathrm{E}+03$ & $3.129 \mathrm{E}-01$ & $1.300 \mathrm{E}+03$ & $9.512 \mathrm{E}-02$ \\
\hline ALCPSO & $4.098 \mathrm{E}+03$ & $5.336 \mathrm{E}+02$ & $1.201 \mathrm{E}+03$ & $5.054 \mathrm{E}-01$ & $1.301 \mathrm{E}+03$ & $8.438 \mathrm{E}-02$ \\
\hline
\end{tabular}


Journal of Bionic Engineering (2021) Vol.18 No.3

\begin{tabular}{|c|c|c|c|c|c|c|}
\hline -DECLS & $5.779 \mathrm{E}+03$ & $3.601 \mathrm{E}+02$ & $1.201 \mathrm{E}+03$ & $1.198 \mathrm{E}-01$ & $1.300 \mathrm{E}+03$ & $5.850 \mathrm{E}-02$ \\
\hline \multirow{2}{*}{ Algorithm } & \multicolumn{2}{|c|}{ F37 } & \multicolumn{2}{|c|}{ F38 } & \multicolumn{2}{|c|}{ F39 } \\
\hline & $\mathrm{AVG}$ & STD & $\mathrm{AVG}$ & STD & $\mathrm{AVG}$ & STD \\
\hline CPA & $1.400 \mathrm{E}+03$ & $1.796 \mathrm{E}-01$ & $1.513 \mathrm{E}+03$ & $7.769 \mathrm{E}+00$ & $1.610 \mathrm{E}+03$ & $6.959 \mathrm{E}-01$ \\
\hline $\mathrm{m} \_\mathrm{SCA}$ & $1.411 \mathrm{E}+03$ & $7.346 \mathrm{E}+00$ & $2.214 \mathrm{E}+03$ & $1.025 \mathrm{E}+03$ & $1.611 \mathrm{E}+03$ & $6.337 \mathrm{E}-01$ \\
\hline OBSCA & $1.460 \mathrm{E}+03$ & $1.124 \mathrm{E}+01$ & $1.426 \mathrm{E}+04$ & $7.328 \mathrm{E}+03$ & $1.613 \mathrm{E}+03$ & $1.389 \mathrm{E}-01$ \\
\hline MWOA & $1.714 \mathrm{E}+03$ & $5.629 \mathrm{E}+01$ & $3.984 \mathrm{E}+06$ & $4.264 \mathrm{E}+06$ & $1.614 \mathrm{E}+03$ & $2.657 \mathrm{E}-01$ \\
\hline IWOA & $1.400 \mathrm{E}+03$ & $1.123 \mathrm{E}-01$ & $1.554 \mathrm{E}+03$ & $1.576 \mathrm{E}+01$ & $1.612 \mathrm{E}+03$ & $4.342 \mathrm{E}-01$ \\
\hline CDLOBA & $1.400 \mathrm{E}+03$ & $1.741 \mathrm{E}-01$ & $1.737 \mathrm{E}+03$ & $8.671 \mathrm{E}+01$ & $1.613 \mathrm{E}+03$ & $4.509 \mathrm{E}-01$ \\
\hline $\mathrm{CBA}$ & $1.400 \mathrm{E}+03$ & $1.188 \mathrm{E}-01$ & $1.562 \mathrm{E}+03$ & $1.798 \mathrm{E}+01$ & $1.613 \mathrm{E}+03$ & $3.217 \mathrm{E}-01$ \\
\hline CGPSO & $1.400 \mathrm{E}+03$ & $1.339 \mathrm{E}-01$ & $1.517 \mathrm{E}+03$ & $1.235 \mathrm{E}+00$ & $1.612 \mathrm{E}+03$ & $3.149 \mathrm{E}-01$ \\
\hline ALCPSO & $1.401 \mathrm{E}+03$ & $3.059 \mathrm{E}-01$ & $1.511 \mathrm{E}+03$ & $3.697 \mathrm{E}+00$ & $1.612 \mathrm{E}+03$ & $4.746 \mathrm{E}-01$ \\
\hline DECLS & $1.400 \mathrm{E}+03$ & $4.454 \mathrm{E}-02$ & $1.512 \mathrm{E}+03$ & $1.303 E+00$ & $1.612 \mathrm{E}+03$ & $2.415 \mathrm{E}-01$ \\
\hline \multirow{2}{*}{ Algorithm } & \multicolumn{2}{|c|}{ F40 } & \multicolumn{2}{|c|}{ F41 } & \multicolumn{2}{|c|}{ F42 } \\
\hline & $\mathrm{AVG}$ & STD & AVG & STD & AVG & STD \\
\hline CPA & $5.600 \mathrm{E}+04$ & $2.684 \mathrm{E}+04$ & $5.088 \mathrm{E}+03$ & $4.764 \mathrm{E}+03$ & $1.908 \mathrm{E}+03$ & $1.339 \mathrm{E}+00$ \\
\hline m_SCA & $1.030 \mathrm{E}+06$ & $5.682 \mathrm{E}+05$ & $2.705 \mathrm{E}+07$ & $3.517 \mathrm{E}+07$ & $1.945 \mathrm{E}+03$ & $2.463 \mathrm{E}+01$ \\
\hline OBSCA & $1.047 \mathrm{E}+07$ & $4.398 \mathrm{E}+06$ & $1.467 \mathrm{E}+08$ & $9.625 \mathrm{E}+07$ & $2.010 \mathrm{E}+03$ & $1.183 \mathrm{E}+01$ \\
\hline MWOA & $2.360 \mathrm{E}+08$ & $1.086 \mathrm{E}+08$ & $6.119 \mathrm{E}+09$ & $2.744 \mathrm{E}+09$ & $2.659 \mathrm{E}+03$ & $3.210 \mathrm{E}+02$ \\
\hline IWOA & $2.149 \mathrm{E}+06$ & $1.481 \mathrm{E}+06$ & $5.974 \mathrm{E}+03$ & $3.608 \mathrm{E}+03$ & $1.943 \mathrm{E}+03$ & $4.172 \mathrm{E}+01$ \\
\hline CDLOBA & $2.362 E+04$ & $1.855 \mathrm{E}+04$ & $7.921 \mathrm{E}+03$ & $6.766 \mathrm{E}+03$ & $1.972 \mathrm{E}+03$ & $4.546 \mathrm{E}+01$ \\
\hline CBA & $2.023 \mathrm{E}+05$ & $1.036 \mathrm{E}+05$ & $1.049 \mathrm{E}+04$ & $9.316 \mathrm{E}+03$ & $1.928 \mathrm{E}+03$ & $2.951 \mathrm{E}+01$ \\
\hline CGPSO & $2.761 \mathrm{E}+05$ & $1.433 \mathrm{E}+05$ & $1.953 \mathrm{E}+06$ & $7.093 \mathrm{E}+05$ & $1.917 \mathrm{E}+03$ & $2.289 \mathrm{E}+00$ \\
\hline ALCPSO & $5.723 \mathrm{E}+05$ & $6.545 \mathrm{E}+05$ & $8.129 \mathrm{E}+03$ & $6.473 \mathrm{E}+03$ & $1.919 \mathrm{E}+03$ & $2.420 \mathrm{E}+01$ \\
\hline DECLS & $1.533 \mathrm{E}+06$ & $8.303 \mathrm{E}+05$ & $1.020 \mathrm{E}+04$ & $6.176 \mathrm{E}+03$ & $1.908 \mathrm{E}+03$ & $7.177 \mathrm{E}-01$ \\
\hline \multirow{2}{*}{ Algorithm } & \multicolumn{2}{|c|}{$\mathrm{F} 43$} & \multicolumn{2}{|c|}{ F44 } & \multicolumn{2}{|c|}{ F45 } \\
\hline & AVG & STD & AVG & STD & AVG & STD \\
\hline CPA & $2.063 \mathrm{E}+03$ & $2.713 \mathrm{E}+01$ & $4.903 \mathrm{E}+04$ & $3.200 \mathrm{E}+04$ & $2.710 \mathrm{E}+03$ & $1.871 \mathrm{E}+02$ \\
\hline $\mathrm{m} \_\mathrm{SCA}$ & $9.629 \mathrm{E}+03$ & $4.457 \mathrm{E}+03$ & $5.113 \mathrm{E}+05$ & $4.819 \mathrm{E}+05$ & $2.561 \mathrm{E}+03$ & $1.718 \mathrm{E}+02$ \\
\hline OBSCA & $2.527 \mathrm{E}+04$ & $7.704 \mathrm{E}+03$ & $1.953 \mathrm{E}+06$ & $1.183 \mathrm{E}+06$ & $3.132 \mathrm{E}+03$ & $1.555 \mathrm{E}+02$ \\
\hline MWOA & $4.700 \mathrm{E}+06$ & $5.323 \mathrm{E}+06$ & $1.122 \mathrm{E}+08$ & $8.287 \mathrm{E}+07$ & $1.599 \mathrm{E}+04$ & $2.983 \mathrm{E}+04$ \\
\hline IWOA & $1.147 \mathrm{E}+04$ & $5.611 \mathrm{E}+03$ & $7.312 \mathrm{E}+05$ & $7.908 \mathrm{E}+05$ & $2.893 \mathrm{E}+03$ & $2.491 \mathrm{E}+02$ \\
\hline CDLOBA & $2.269 \mathrm{E}+04$ & $1.259 \mathrm{E}+04$ & $2.837 E+04$ & $1.657 \mathrm{E}+04$ & $3.423 \mathrm{E}+03$ & $3.906 \mathrm{E}+02$ \\
\hline $\mathrm{CBA}$ & $2.555 \mathrm{E}+03$ & $3.179 \mathrm{E}+02$ & $7.348 \mathrm{E}+04$ & $4.454 \mathrm{E}+04$ & $3.430 \mathrm{E}+03$ & $3.740 \mathrm{E}+02$ \\
\hline CGPSO & $2.343 \mathrm{E}+03$ & $5.773 \mathrm{E}+01$ & $1.264 \mathrm{E}+05$ & $7.996 \mathrm{E}+04$ & $2.855 \mathrm{E}+03$ & $2.061 \mathrm{E}+02$ \\
\hline ALCPSO & $2.556 \mathrm{E}+03$ & $2.462 \mathrm{E}+02$ & $5.603 \mathrm{E}+04$ & $4.688 \mathrm{E}+04$ & $2.662 \mathrm{E}+03$ & $1.771 \mathrm{E}+02$ \\
\hline DECLS & $4.568 \mathrm{E}+03$ & $1.639 \mathrm{E}+03$ & $3.425 \mathrm{E}+05$ & $1.514 \mathrm{E}+05$ & $2.387 \mathrm{E}+03$ & $8.056 \mathrm{E}+01$ \\
\hline \multirow{2}{*}{ Algorithm } & \multicolumn{2}{|c|}{$\mathrm{F} 46$} & \multicolumn{2}{|c|}{ F47 } & \multicolumn{2}{|c|}{$\mathrm{F} 48$} \\
\hline & AVG & STD & AVG & STD & AVG & STD \\
\hline CPA & $2.500 \mathrm{E}+03$ & $0.000 \mathrm{E}+00$ & $2.600 \mathrm{E}+03$ & $0.000 \mathrm{E}+00$ & $2.700 \mathrm{E}+03$ & $0.000 \mathrm{E}+00$ \\
\hline $\mathrm{m}_{-} \mathrm{SCA}$ & $2.639 \mathrm{E}+03$ & $9.903 \mathrm{E}+00$ & $2.600 \mathrm{E}+03$ & $6.768 \mathrm{E}-04$ & $2.714 \mathrm{E}+03$ & $4.213 \mathrm{E}+00$ \\
\hline OBSCA & $2.680 \mathrm{E}+03$ & $1.589 \mathrm{E}+01$ & $2.600 \mathrm{E}+03$ & $1.469 \mathrm{E}-04$ & $2.700 \mathrm{E}+03$ & $5.278 \mathrm{E}-04$ \\
\hline MWOA & $3.757 \mathrm{E}+03$ & $3.789 \mathrm{E}+02$ & $2.808 \mathrm{E}+03$ & $3.914 \mathrm{E}+01$ & $2.828 \mathrm{E}+03$ & $4.214 \mathrm{E}+01$ \\
\hline IWOA & $2.618 \mathrm{E}+03$ & $1.739 \mathrm{E}+00$ & $2.602 \mathrm{E}+03$ & $1.354 \mathrm{E}+00$ & $2.715 \mathrm{E}+03$ & $1.430 \mathrm{E}+01$ \\
\hline CDLOBA & $2.615 \mathrm{E}+03$ & $3.789 \mathrm{E}-01$ & $2.713 \mathrm{E}+03$ & $5.314 \mathrm{E}+01$ & $2.722 \mathrm{E}+03$ & $1.233 \mathrm{E}+01$ \\
\hline $\mathrm{CBA}$ & $2.616 \mathrm{E}+03$ & $1.430 \mathrm{E}-01$ & $2.682 \mathrm{E}+03$ & $3.643 \mathrm{E}+01$ & $2.737 \mathrm{E}+03$ & $1.336 \mathrm{E}+01$ \\
\hline CGPSO & $2.500 \mathrm{E}+03$ & $9.389 \mathrm{E}-04$ & $2.600 \mathrm{E}+03$ & $9.499 \mathrm{E}-03$ & $2.700 \mathrm{E}+03$ & $1.505 \mathrm{E}-05$ \\
\hline ALCPSO & $2.615 \mathrm{E}+03$ & $5.937 \mathrm{E}-02$ & $2.639 \mathrm{E}+03$ & $7.224 \mathrm{E}+00$ & $2.711 \mathrm{E}+03$ & $4.146 \mathrm{E}+00$ \\
\hline
\end{tabular}




\begin{tabular}{|c|c|c|c|c|c|c|c|}
\hline DECLS & $2.500 E+03$ & $6.901 \mathrm{E}-04$ & $2.600 E+03$ & $7.578 \mathrm{E}-03$ & \multicolumn{2}{|c|}{$2.700 E+03$} & $7.747 \mathrm{E}-06$ \\
\hline \multirow{2}{*}{ Algorithm } & \multicolumn{2}{|c|}{ F49 } & \multicolumn{2}{|c|}{ F50 } & \multicolumn{3}{|c|}{ F51 } \\
\hline & AVG & STD & AVG & STD & \multicolumn{2}{|c|}{ AVG } & STD \\
\hline CPA & $2.701 \mathrm{E}+03$ & $1.447 \mathrm{E}-01$ & $2.900 \mathrm{E}+03$ & $0.000 E+00$ & \multicolumn{2}{|c|}{$3.000 E+03$} & $0.000 \mathrm{E}+00$ \\
\hline m_SCA & $2.701 \mathrm{E}+03$ & $3.736 \mathrm{E}-01$ & $3.151 \mathrm{E}+03$ & $6.149 \mathrm{E}+01$ & \multicolumn{2}{|c|}{$3.876 \mathrm{E}+03$} & $1.196 \mathrm{E}+02$ \\
\hline OBSCA & $2.704 \mathrm{E}+03$ & $5.210 \mathrm{E}-01$ & $3.224 \mathrm{E}+03$ & $4.059 \mathrm{E}+01$ & \multicolumn{2}{|c|}{$5.456 \mathrm{E}+03$} & $3.081 \mathrm{E}+02$ \\
\hline MWOA & $2.764 \mathrm{E}+03$ & $8.949 \mathrm{E}+01$ & $4.408 \mathrm{E}+03$ & $2.019 \mathrm{E}+02$ & \multicolumn{2}{|c|}{$8.187 \mathrm{E}+03$} & $1.082 \mathrm{E}+03$ \\
\hline IWOA & $2.701 \mathrm{E}+03$ & $1.489 \mathrm{E}-01$ & $3.582 \mathrm{E}+03$ & $3.113 \mathrm{E}+02$ & \multicolumn{2}{|c|}{$4.689 \mathrm{E}+03$} & $5.042 \mathrm{E}+02$ \\
\hline CDLOBA & $2.704 \mathrm{E}+03$ & $1.814 \mathrm{E}+01$ & $3.727 \mathrm{E}+03$ & $4.539 \mathrm{E}+02$ & \multicolumn{2}{|c|}{$5.456 \mathrm{E}+03$} & $7.152 \mathrm{E}+02$ \\
\hline CBA & $2.722 \mathrm{E}+03$ & $8.145 \mathrm{E}+01$ & $3.969 \mathrm{E}+03$ & $4.524 \mathrm{E}+02$ & \multicolumn{2}{|c|}{$5.536 \mathrm{E}+03$} & $6.722 \mathrm{E}+02$ \\
\hline CGPSO & $2.800 \mathrm{E}+03$ & $1.346 \mathrm{E}-06$ & $3.145 \mathrm{E}+03$ & $3.610 \mathrm{E}+02$ & \multicolumn{2}{|c|}{$3.000 \mathrm{E}+03$} & $2.588 \mathrm{E}-02$ \\
\hline ALCPSO & $2.755 \mathrm{E}+03$ & $6.117 \mathrm{E}+01$ & $3.452 \mathrm{E}+03$ & $2.301 \mathrm{E}+02$ & \multicolumn{2}{|c|}{$4.472 \mathrm{E}+03$} & $5.191 \mathrm{E}+02$ \\
\hline DECLS & $2.700 \mathrm{E}+03$ & $5.098 \mathrm{E}-02$ & $3.047 \mathrm{E}+03$ & $1.913 \mathrm{E}+02$ & \multicolumn{2}{|c|}{$3.021 \mathrm{E}+03$} & $1.176 \mathrm{E}+02$ \\
\hline \multirow{2}{*}{ Algorithm } & \multicolumn{2}{|c|}{ F52 } & \multicolumn{2}{|c|}{ F53 } & \multirow[b]{2}{*}{$+/-1=$} & \multirow[b]{2}{*}{ Avg } & \multirow[b]{2}{*}{ Rank } \\
\hline & AVG & STD & AVG & STD & & & \\
\hline CPA & $3.100 \mathrm{E}+03$ & $0.000 E+00$ & $3.200 \mathrm{E}+03$ & $0.000 \mathrm{E}+00$ & $\sim$ & 2.88 & 1 \\
\hline m_SCA & $8.126 \mathrm{E}+05$ & $2.707 \mathrm{E}+06$ & $4.283 \mathrm{E}+04$ & $1.997 \mathrm{E}+04$ & $44 / 2 / 7$ & 6.11 & 6 \\
\hline OBSCA & $1.209 \mathrm{E}+07$ & $6.124 \mathrm{E}+06$ & $3.712 \mathrm{E}+05$ & $1.448 \mathrm{E}+05$ & $47 / 1 / 5$ & 7.97 & 9 \\
\hline MWOA & $2.509 \mathrm{E}+08$ & $1.549 \mathrm{E}+08$ & $5.695 \mathrm{E}+06$ & $3.390 \mathrm{E}+06$ & $53 / 0 / 0$ & 10.76 & 10 \\
\hline IWOA & $5.345 \mathrm{E}+06$ & $4.456 \mathrm{E}+06$ & $2.079 \mathrm{E}+04$ & $1.007 \mathrm{E}+04$ & $42 / 2 / 9$ & 5.68 & 4 \\
\hline CDLOBA & $1.741 \mathrm{E}+07$ & $1.711 \mathrm{E}+07$ & $1.314 \mathrm{E}+05$ & $2.196 \mathrm{E}+05$ & $42 / 5 / 6$ & 7.63 & 8 \\
\hline CBA & $4.239 \mathrm{E}+07$ & $4.060 \mathrm{E}+07$ & $6.782 \mathrm{E}+04$ & $1.472 \mathrm{E}+05$ & $47 / 1 / 5$ & 7.25 & 7 \\
\hline CGPSO & $4.138 \mathrm{E}+03$ & $9.991 \mathrm{E}+02$ & $1.009 \mathrm{E}+04$ & $9.652 \mathrm{E}+03$ & $44 / 6 / 3$ & 6.11 & 5 \\
\hline ALCPSO & $3.452 \mathrm{E}+06$ & $1.169 \mathrm{E}+07$ & $1.130 \mathrm{E}+04$ & $6.340 \mathrm{E}+03$ & $33 / 6 / 14$ & 4.86 & 3 \\
\hline DECLS & $4.911 \mathrm{E}+03$ & $8.387 \mathrm{E}+02$ & $6.763 \mathrm{E}+03$ & $8.839 \mathrm{E}+02$ & $27 / 14 / 12$ & 3.72 & 2 \\
\hline
\end{tabular}

\section{1 Tension-compression spring problem}

The mathematical model of a Tension/Compression Spring (TCS) design is designed to minimize the weight of the spring. This problem requires the optimization of three design variables, including the wire diameter $(d)$, mean coil diameter $(D)$, and number of active coils $(N)$. Fig. 9 illustrates this problem.

The mathematical model of this problem is as follows:

Consider $\stackrel{\mathrm{r}}{x}=\left[\begin{array}{lll}x_{1} & x_{2} & x_{3}\end{array}\right]=\left[\begin{array}{lll}d & D & N\end{array}\right]$

Objective function:

$$
f(x)_{\min }=x_{1}^{2} x_{2} x_{3}+2 x_{1}^{2} x_{2}
$$

Subject to

$$
\begin{aligned}
& h_{1}(\stackrel{r}{x})=1-\frac{x_{2}^{3} x_{3}}{71785 x_{1}^{4}} \leq 0 \\
& h_{2}(\stackrel{r}{x})=\frac{4 x_{2}^{2}-x_{1} x_{2}}{12566\left(x_{2} x_{1}^{3}-x_{1}^{4}\right)}+\frac{1}{5180 x_{1}^{2}} \leq 0
\end{aligned}
$$

$$
\begin{aligned}
& h_{3}(\stackrel{\mathrm{r}}{x})=1-\frac{140.45 x_{1}}{x_{2}{ }^{3} x_{3}} \leq 0 \\
& h_{4}(\stackrel{\mathrm{r}}{x})=\frac{x_{1}+x_{2}}{1.5}-1 \leq 0
\end{aligned}
$$

Variable ranges:

$$
\begin{aligned}
& 0.05 \leq x_{1} \leq 1.00 \\
& 0.25 \leq x_{2} \leq 1.30 \\
& 2.00 \leq x_{3} \leq 15.0
\end{aligned}
$$

The model has been solved by mathematical optimization tools and metaheuristic methods. For instance, Coello et al. realized this problem by using Genetic Algorithms (GA) and obtained an adaptation value of 0.0127048 . The experimental results, after testing many algorithms, show that the optimal weight can reach 0.0126650 .

Table 5 reveals that the weight calculated by CPA is smaller than the current optimal weight, which also proves the superiority of the CPA in TCSD. 

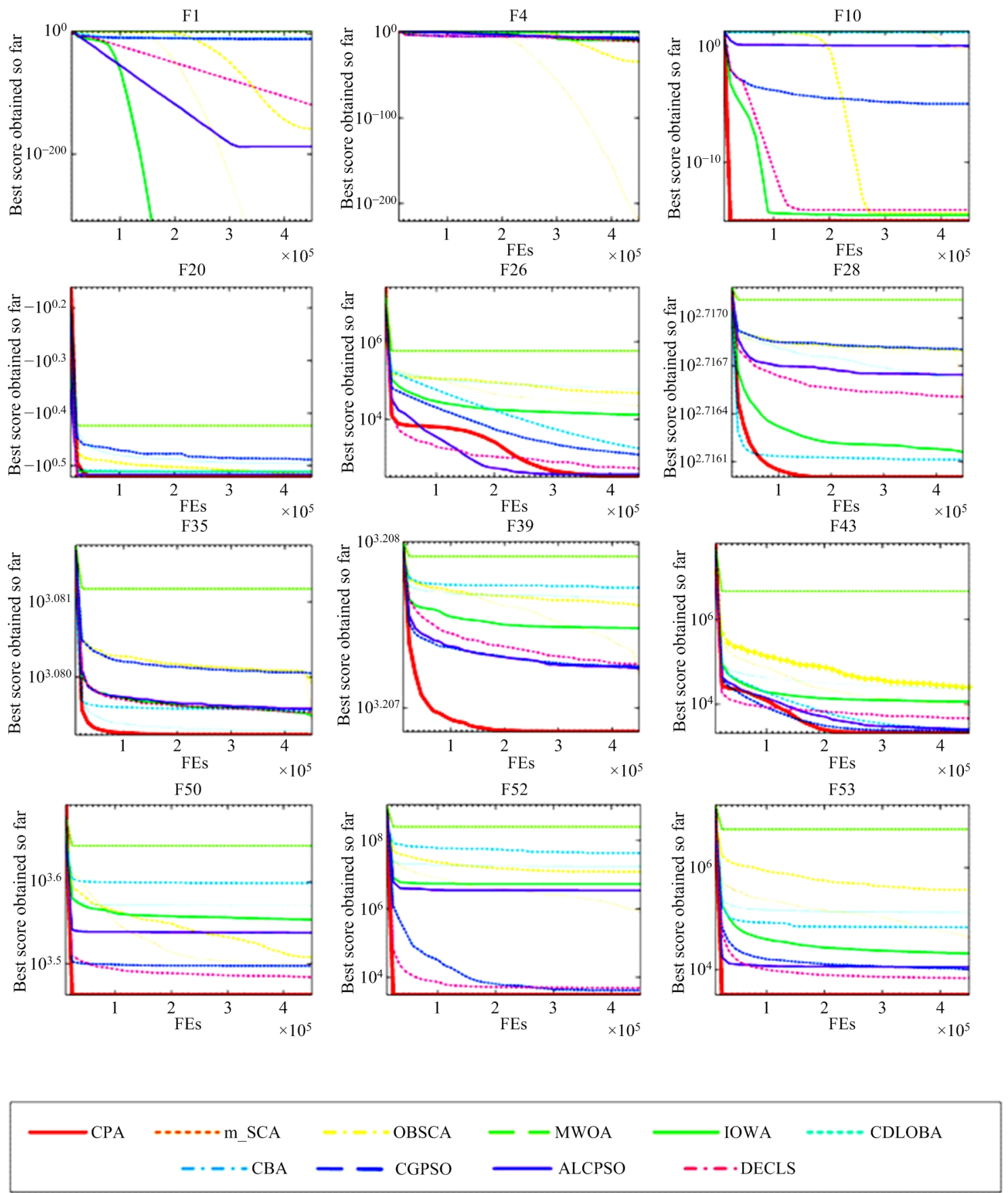

Fig. 7 Comparisons between CPA and advanced MAs.

\section{2 Welded beam design problem}

The purpose of this problem is to find the lowest cost of welded beams under four constraints; shear stress $(\tau)$, bending stress $(\theta)$, buckling load $\left(P_{C}\right)$, and deflection $(\delta)$. These four variables of this problem include the welding seam thickness $(h)$, welding joint length, beam width $(t)$, and beam thickness $(b)$. Fig. 10 demonstrates the elements of this problem.

The mathematical model is as follows:

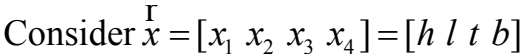

Minimize

$$
f(x)_{\min }=1.10471 x_{1}^{2}+0.04811 x_{3} x_{4}\left(14.0+x_{4}\right)
$$

Subject to $g_{1}(\stackrel{\mathrm{r}}{x})=\tau(\stackrel{\mathrm{r}}{x})-\tau_{\max } \leq 0$ 
Table 3 Ranking results of upper limits for different $S$

\begin{tabular}{cccccccccccc}
\hline Fun & $a$ & $2 a / 3$ & $a / 2$ & $a / 3$ & $a / 4$ & $a / 5$ & $a / 6$ & $a / 7$ & $a / 8$ & $a / 9$ & $a / 10$ \\
\hline ARV & 6.7358 & 3.00 & 3.8490 & 4.5094 & 4.9811 & 4.8490 & 4.7547 & 5.9622 & 6.0566 & 6.00 & 6.1509 \\
Rank & 11 & 1 & 2 & 3 & 6 & 5 & 4 & 7 & 9 & 8 & 10 \\
\hline
\end{tabular}

Table 4 Ranking of results with different values of parameter $w$

\begin{tabular}{ccccccccccc}
\hline Fun & $w=1$ & $w=2$ & $w=3$ & $w=4$ & $w=5$ & $w=6$ & $w=7$ & $w=8$ & $w=9$ & $w=10$ \\
\hline ARV & 6.30 & 6.00 & 5.71 & 5.56 & 5.45 & 5.33 & 5.18 & 5.20 & 5.06 & 5.13 \\
Rank & 10 & 9 & 8 & 7 & 6 & 5 & 4 & 3 & 1 & 2 \\
\hline
\end{tabular}

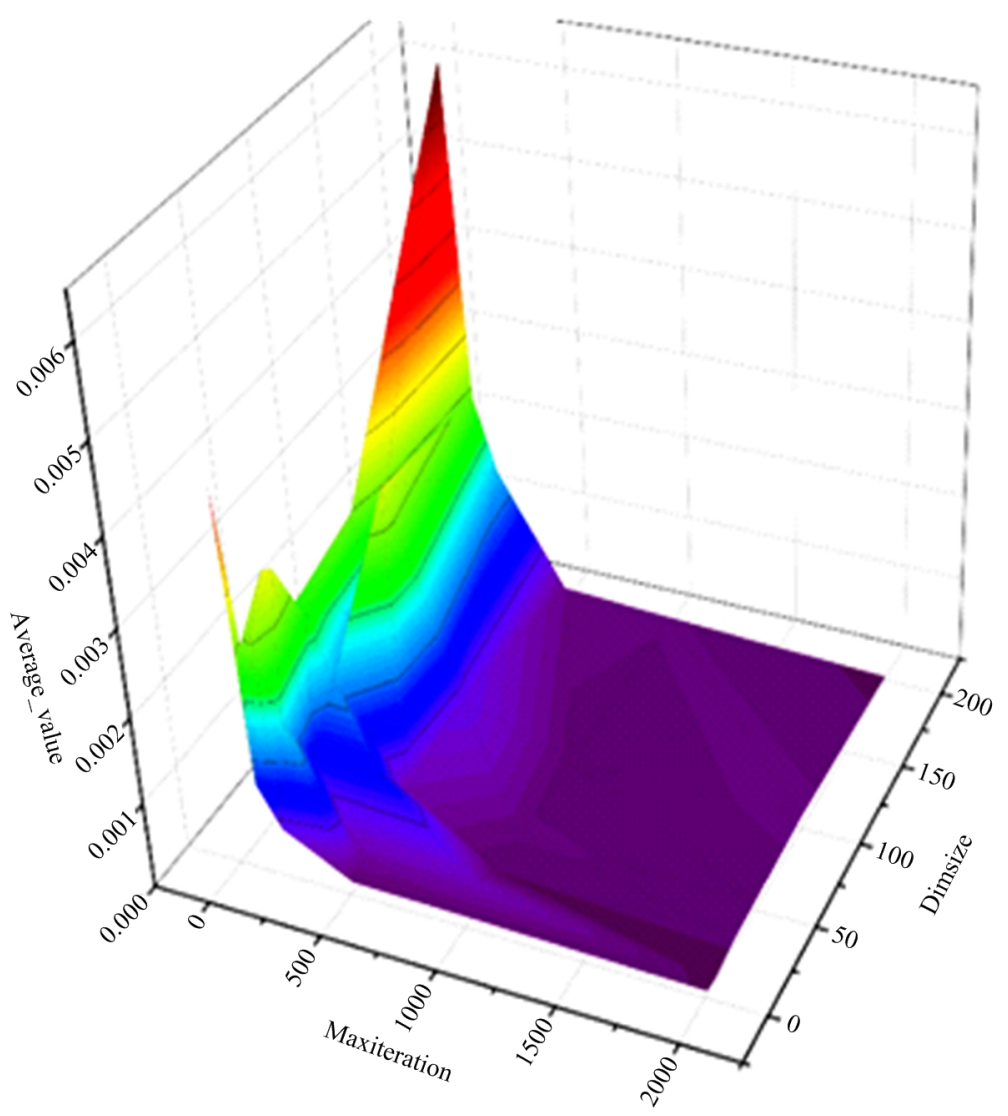

Average_value

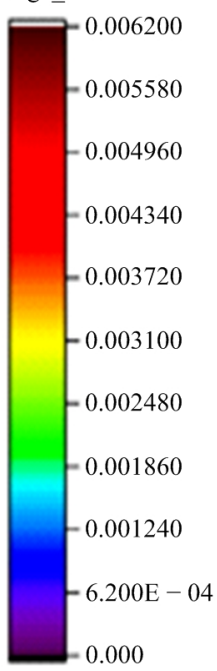

Fig. 8 The influence of $N$ and Max_iter.

$$
\begin{aligned}
& g_{2}(\stackrel{r}{x})=\sigma(\stackrel{r}{x})-\sigma_{\max } \leq 0 \\
& g_{3}(\stackrel{r}{x})=\delta(\stackrel{r}{x})-\delta_{\max } \leq 0 \\
& g_{4}(\stackrel{r}{x})=x_{1}-x_{4} \leq 0 \\
& g_{5}(\stackrel{r}{x})=P-P_{c}(\stackrel{r}{x}) \leq 0 \\
& g_{6}(\stackrel{r}{x})=0.125-x_{1} \leq 0 \\
& g_{7}(\stackrel{r}{x})=1.10471 x_{1}^{2}+0.04811 x_{3} x_{4}\left(14.0+x_{4}\right)-5.0 \leq 0
\end{aligned}
$$$$
0.1 \leq x_{4} \leq 2
$$$$
\text { where } \quad \tau(\stackrel{\mathrm{r}}{x})=\sqrt{\left(\tau^{\prime}\right)^{2}+2 \tau^{\prime} \tau^{\prime \prime} \frac{x_{2}}{2 R}+\left(\tau^{\prime \prime}\right)^{2}} \quad \tau^{\prime}=\frac{P}{\sqrt{2} x_{1} x_{2}}
$$$$
\tau^{\prime \prime}=\frac{M R}{J} M=P\left(L+\frac{x_{2}}{2}\right)
$$$$
R=\sqrt{\frac{x_{2}^{2}}{4}+\left(\frac{x_{1}+x_{3}}{2}\right)^{2}}
$$

Variable range $0.1 \leq x_{1} \leq 2,0.1 \leq x_{2} \leq 10,0.1 \leq x_{3} \leq 10$

$$
J=2\left\{\sqrt{2} x_{1} x_{2}\left[\frac{x_{2}^{2}}{4}+\left(\frac{x_{1}+x_{3}}{2}\right)^{2}\right]\right\}
$$




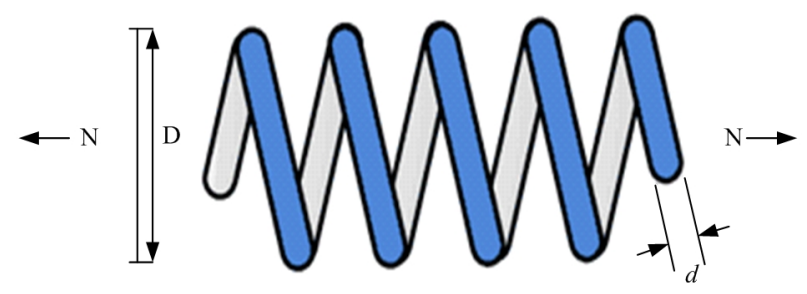

Fig. 9 The structure of the tension-compression spring.

Table 5 Results of CPA versus other algorithms for TCS design problem

\begin{tabular}{ccccc}
\hline \multirow{2}{*}{ Algorithm } & \multicolumn{3}{c}{ Optimal values for variables } & \multirow{2}{*}{$\begin{array}{c}\text { Optimum } \\
\text { cost }\end{array}$} \\
\cline { 2 - 4 } & $d$ & $D$ & $N$ & $\mathbf{0 . 0 1 2 6 6 5 0}$ \\
CPA & $\mathbf{0 . 0 5 1 7 4 1}$ & $\mathbf{0 . 3 5 7 9 7 8}$ & $\mathbf{1 1 . 2 1 5 4 8 0}$ & $\mathbf{0 . 0 1 2 6}$ \\
GA & 0.051480 & 0.351661 & 11.632201 & 0.0127048 \\
RO $^{[86]}$ & 0.051370 & 0.349096 & 11.762790 & 0.0126788 \\
IHS $^{[87]}$ & 0.051154 & 0.349871 & 12.076432 & 0.0126706 \\
$\begin{array}{l}\text { Constraint correction } \\
\text { method (Arora) }\end{array}$ & 0.050000 & 0.315900 & 14.250000 & 0.0128334 \\
$\begin{array}{l}\text { Mathematical optimi- } \\
\text { zation method (Bele- } \\
\text { gundu) }\end{array}$ & 0.053396 & 0.399180 & 901854000 & 0.0127303 \\
WOA & 0.051207 & 0.345215 & 12.0043032 & 0.0126763 \\
PSO & 0.015728 & 0.357644 & 11.244543 & 0.0126747 \\
\hline
\end{tabular}

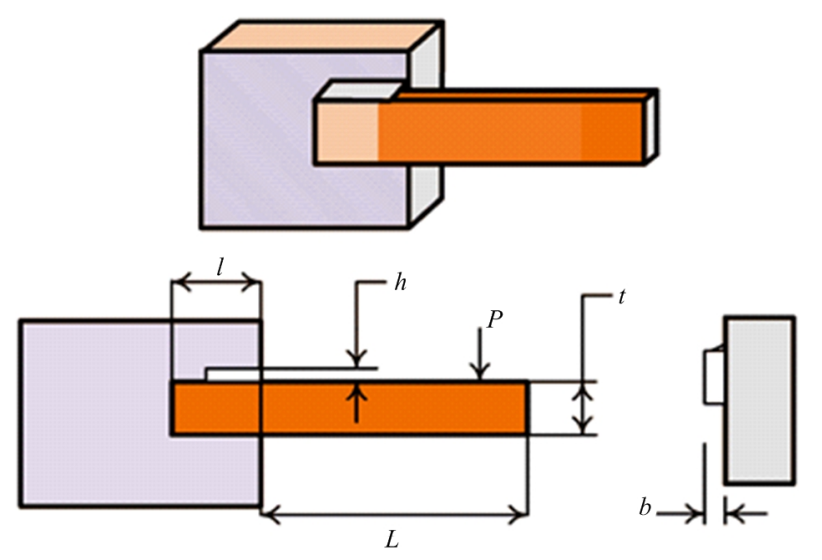

Fig. 10 The structure of a welded beam.

Table 6 Results compared with other approaches

\begin{tabular}{cccccc}
\hline \multirow{2}{*}{ Algorithm } & \multicolumn{4}{c}{ Optimal values for variables } & Optimum \\
\cline { 2 - 5 } & $h$ & $l$ & $t$ & $b$ & cost \\
\hline CPA & $\mathbf{0 . 1 8 8 5 0 0}$ & $\mathbf{3 . 5 6 2 0 0 0}$ & $\mathbf{9 . 1 3 4 8 3 5}$ & $\mathbf{0 . 2 0 5 2 4 5}$ & $\mathbf{1 . 7 2 3 9 1 6}$ \\
RO & 0.203687 & 3.528467 & 9.004233 & 0.207241 & 1.735344 \\
SSA & 0.205700 & 3.471400 & 9.036600 & 0.205700 & 1.724910 \\
CDE & 0.203137 & 3.542998 & 9.033498 & 0.206179 & 1.733462 \\
GWO & 0.205700 & 3.478400 & 9.036800 & 0.205800 & 1.726240 \\
WOA & 0.175500 & 3.893500 & 9.018932 & 0.206538 & 1.736036 \\
GSA & 0.182100 & 3.857000 & 10.00000 & 0.202400 & 1.879950 \\
\hline
\end{tabular}

$$
\begin{aligned}
& \sigma(\stackrel{\mathrm{r}}{x})=\frac{6 P L}{x_{4} x_{3}^{2}}, \delta(\stackrel{\mathrm{r}}{x})=\frac{6 P L^{3}}{E x_{4} x_{3}^{2}} \\
& P_{C}(\stackrel{\mathrm{r}}{x})=\frac{4.013 E \sqrt{\frac{x_{4}{ }^{6} x_{3}^{2}}{36}}}{L^{2}}\left(1-\frac{x_{3}}{2 L} \sqrt{\frac{E}{4 G}}\right) \\
& P_{C}=60001 b, L=14, \delta_{\text {max }}=0.25 \\
& E=30 \times 1^{6} p s i, G=12 \times 10^{6} p s i \\
& \tau_{\max }=13600 \mathrm{psi}, \sigma_{\text {max }}=30000 \mathrm{psi}
\end{aligned}
$$

The performance of CPA was compared with those of RO, SSA, $\mathrm{CDE}^{[88]}, \mathrm{GWO}, \mathrm{WOA}$, and GSA are compared for this problem. The current optimal weight is 1.72452 . We can find from Table 6 that the SSA has the best performance, achieving a weight of 1.723916 .

\section{3 Pressure vessel design problem}

This problem is considered a well-studied structural optimization test case, which aims to minimize the production cost of cylindrical pressure vessels. One end of the container is covered, and the other end is a hemisphere, for which the total manufacturing costs is determined by four variables - the thickness of the shell $T_{s}$, the thickness of the head $\left(T_{h}\right)$, inner radius $(R)$, and the span of the cylindrical part of the container $(L)-$ determine the total manufacturing cost. Fig. 11 contains an illustration of this problem.

The relevant formulas are as follows:

Consider $\stackrel{\mathrm{r}}{x}=\left[\begin{array}{llll}x_{1} & x_{2} & x_{3} & x_{4}\end{array}\right]=\left[\begin{array}{llll}T_{s} & T_{h} & R & L\end{array}\right]$

Minimize

$$
\begin{aligned}
& f(\stackrel{\mathrm{r}}{x})_{\min }=0.6224 x_{1} x_{3} x_{4}+1.7781 x_{3} x_{1}^{2}+3.1661 x_{4} x_{1}^{2} \\
& +19.84 x_{3} x_{1}^{2} \\
& g_{2}(\stackrel{r}{x})=-x_{2}+0.0095 x_{3} \leq 0
\end{aligned}
$$

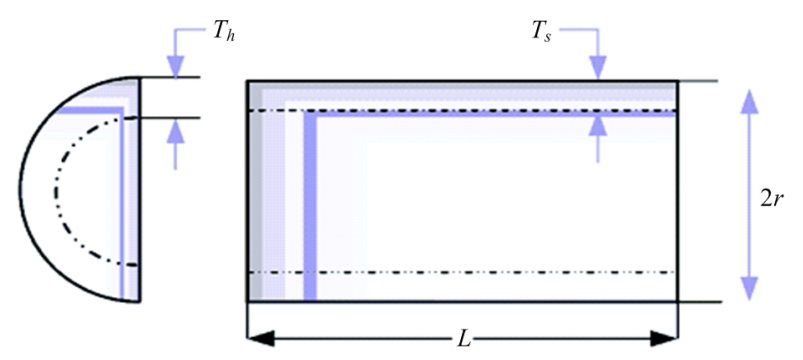

Fig. 11 The structure of the pressure vessel. 
Table 7 Results of pressure vessel design problem compared with other methods

\begin{tabular}{cccccc}
\hline Algorithm & \multicolumn{2}{c}{ Optimal values for variables } & \multicolumn{1}{c}{ Optimum cost } \\
\cline { 2 - 5 } & $T_{S}$ & $T_{h}$ & 42.088230 & 176.763300 & 6060.9590 \\
CPA & 0.81250 & 0.437500 & 42.091266 & 176.746500 & 6061.0777 \\
PSO & 0.812500 & 0.437500 & $\mathbf{4 2 . 0 9 8 4 0 0}$ & $\mathbf{1 7 6 . 6 3 6 6 0 0}$ & $\mathbf{6 0 5 9 . 7 1 4 3}$ \\
MFO & $\mathbf{0 . 8 1 2 5 0 0}$ & $\mathbf{0 . 4 3 7 5 0 0}$ & 42.089200 & 176.758700 & 6051.5639 \\
GWO & 0.812500 & 0.434500 & 42.098300 & 176.639000 & 6059.7410 \\
WOA & 0.812500 & 0.437500 & $\mathbf{4 2 . 0 9 8 4 4 5}$ & $\mathbf{1 7 6 . 6 3 6 5 9 5}$ & $\mathbf{6 0 5 9 . 7 1 4 3}$ \\
BA & $\mathbf{0 . 8 1 2 5 0 0}$ & $\mathbf{0 . 4 3 7 5 0 0}$ & 42.091266 & 176.746500 & 6061.0777 \\
CPSO & 0.812500 & 0.437500 & $\mathbf{4 2 . 0 9 8 4 0 0}$ & $\mathbf{1 7 6 . 6 3 6 6 0 0}$ & $\mathbf{6 0 5 9 . 7 1 4 3}$ \\
HPSO & $\mathbf{0 . 8 1 2 5 0 0}$ & $\mathbf{0 . 4 3 7 5 0 0}$ & 58.291000 & 43.6900000 & 7198.0428 \\
Lagrangian multiplier & 1.125000 & 0.625000 & 47.700000 & 117.701000 & 8129.1036 \\
Branch-bound & 1.125000 & 0.625000 &
\end{tabular}

Table 8 Results of I-beam design problem compared with other methods

\begin{tabular}{cccccc}
\hline \multirow{2}{*}{ Algorithm } & \multicolumn{5}{c}{ Optimal values for variables } \\
\cline { 2 - 5 } & $B$ & $h$ & $T_{w}$ & $T_{f}$ & Optimum cost \\
\hline CPA & 49.9141 & 79.8044 & 0.935684 & 2.300281 & 0.0132240 \\
ARSM & 48.4200 & 79.9900 & 0.900000 & 2.400000 & 0.0157000 \\
IARSM & 48.4200 & 79.9900 & 0.900000 & 2.400000 & 0.1310000 \\
CS & 50.0000 & 80.0000 & 0.900000 & 2.321675 & 0.0130747 \\
SOS & $\mathbf{5 0 . 0 0 0 0}$ & $\mathbf{8 0 . 0 0 0 0}$ & $\mathbf{0 . 9 0 0 0 0 0}$ & $\mathbf{2 . 3 2 1 7 9 0}$ & $\mathbf{0 . 0 1 3 0 7 4 1}$ \\
\hline
\end{tabular}
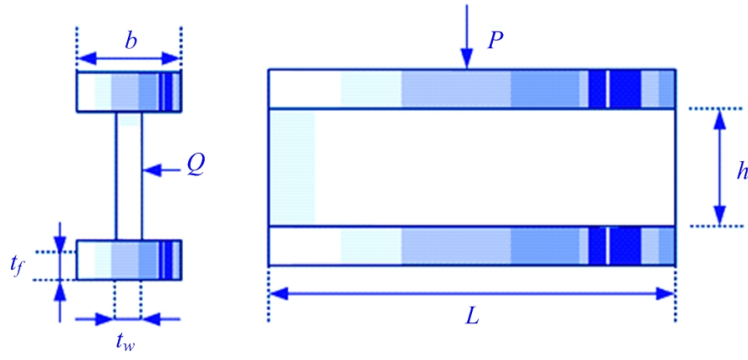

Fig. 12 The structure of I-beam.

$$
\begin{aligned}
& g_{3}(\stackrel{r}{x})=-\pi x_{4} x_{3}{ }^{2}+\frac{4}{3} \pi x_{3}^{3}+1296000 \leq 0 \\
& g_{4}(\stackrel{r}{x})=x_{4}-240 \leq 0
\end{aligned}
$$

Variable range

$0 \leq x_{1} \leq 99,0 \leq x_{2} \leq 99,10 \leq x_{3} \leq 200,10 \leq x_{4} \leq 200$

For this engineering problem, this work compared the performance of CPA with those of PSO, MFO, GWO, WOA, BA, $\mathrm{CPSO}^{[89]}$, $\mathrm{HPSO}^{[90]}$, Lagrangian multiplier ${ }^{[91]}$ and Branch-bound ${ }^{[92]}$. Table 7 shows that the optimal value of the CPA was about 1.2 higher than those of MFO, BA, and HPSO. Thus, the latter three MAs achieved minimum optimal consumption, but still exceeded the current optimal weight of 6059.7143 .

\section{4 I-beam design problem}

The purpose of this problem is to minimize the vertical deflection of the I-beams. Related parameters include the length, height, and two thicknesses. Fig. 12 shows the configuration of this problem.

The mathematical model of the problem is as follows:

Consider $\quad \stackrel{\mathrm{r}}{x}=\left[\begin{array}{llll}x_{1} & x_{2} & x_{3} & x_{4}\end{array}\right]=\left[\begin{array}{lll}b & h & t_{w}\end{array} t_{f}\right]$

Objective

$$
\begin{gathered}
f(\stackrel{\mathrm{r}}{x})_{\min }=\frac{5000}{\frac{t_{w}\left(h-2 t_{f}\right)^{3}}{12}+\frac{b t_{f}{ }^{3}}{6}+2 b t_{f}\left(\frac{h-t_{f}}{2}\right)^{2}} \\
\text { Subject to } \quad g_{1}(x)=2 b t_{w}+t_{w}\left(h-2 t_{f}\right) \leq 0 \\
g_{1}(\stackrel{\mathrm{r}}{x})=\frac{18 h \times 10^{4}}{t_{w}\left(h-2 t_{f}\right)^{3}+2 b t_{f}\left(4 t_{f}+3 h\left(h-2 t_{f}\right)\right)} \\
+\frac{15 b \times 10^{3}}{\left(h-2 t_{f}\right) t_{w}{ }^{3}+2 t_{f} b^{3}}
\end{gathered}
$$

Variable range $10 \leq x_{1} \leq 50$

$$
\begin{aligned}
& 10 \leq x_{2} \leq 80 \\
& 0.9 \leq x_{3} \leq 5 \\
& 0.9 \leq x_{4} \leq 5
\end{aligned}
$$

Table 8 is a comparison between CPA and 
Table 9 Results of Multiple disk clutch brake compared with other methods

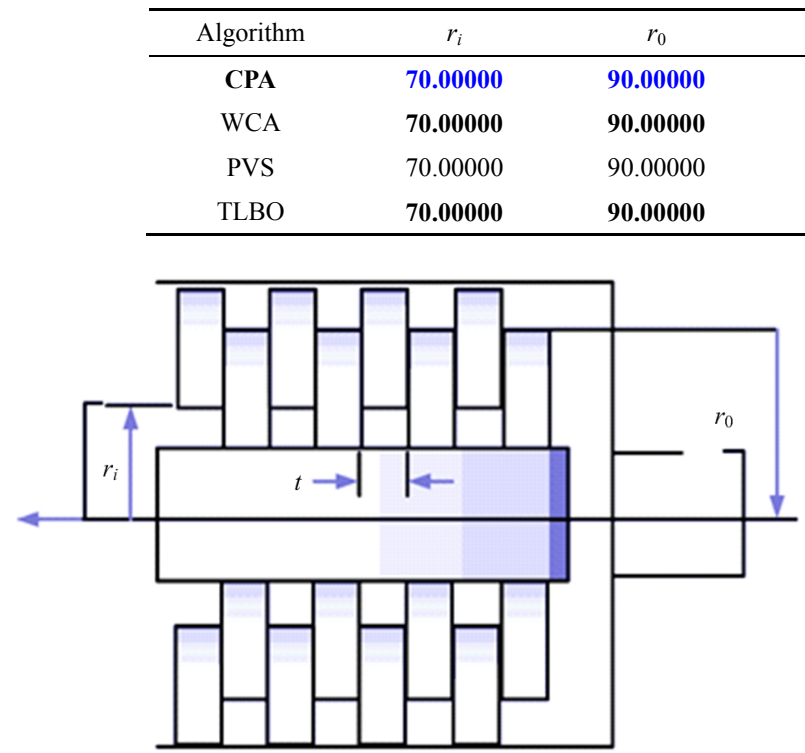

Fig. 13 The structure of multiple disk clutch brake.

$\mathrm{ARSM}^{[93]}, \mathrm{IARSM}^{[93]}, \mathrm{CS}$, and $\mathrm{SOS}^{[94]}$ on I-beam problems. We can find from Table 8 that CPA can help the vertical deflection of the I-beam to minimize more than the other four algorithms, which is the same as the current optimal value of 0.006626 , which shows that $\mathrm{CPA}$ is more suitable for engineering problems.

\section{5 Multiple disk clutch brake problem}

The objective of this minimization and discrete optimization problem is to use five discrete design variables to minimize the quality of multiple disc clutch brakes. The five variables are the actuating force, inner and outer radii, number of 27 friction surfaces, and thickness of discs. Fig. 13 shows the configuration of this problem.

The mathematical model for this problem is as follows:

$$
f(\stackrel{\mathrm{r}}{x})_{\min }=\pi\left(r_{0}^{2}-r_{i}^{2}\right) t(Z+1) \rho
$$

subject to:

$$
\begin{aligned}
& g_{1}(\stackrel{\mathrm{r}}{x})=r_{0}-r_{i}-\Delta r \geq 0 \\
& g_{2}(\stackrel{\mathrm{r}}{x})=l_{\text {max }}-(Z+1)(t+\delta) \geq 0 \\
& g_{3}(\stackrel{\mathrm{r}}{x})=P_{\max }-P_{r z} \geq 0 \\
& g_{4}(\stackrel{\mathrm{r}}{x})=P_{\text {max }} v_{s r_{\max }}-P_{r z} v_{s r} \geq 0
\end{aligned}
$$

\begin{tabular}{ccc}
$F$ & $Z$ & Optimal cost \\
$\mathbf{9 9 0 0 0}$ & $\mathbf{3 . 0 0 0 0 0}$ & $\mathbf{0 . 3 1 3 6 5 6}$ \\
$\mathbf{9 1 0 . 0 0 0 0}$ & $\mathbf{3 . 0 0 0 0 0 0}$ & $\mathbf{0 . 3 1 3 6 5 6}$ \\
980.0000 & 3.000000 & 0.313660 \\
$\mathbf{8 1 0 . 0 0 0 0}$ & $\mathbf{3 . 0 0 0 0 0 0}$ & $\mathbf{0 . 3 1 3 6 5 6}$ \\
\hline
\end{tabular}

$$
\begin{aligned}
& g_{5}(\stackrel{r}{x})=v_{s r_{\max }}-v_{s r} \geq 0 \\
& g_{6}(\stackrel{\mathrm{r}}{x})=T_{\text {max }}-T \geq 0 \\
& g_{7}(\stackrel{\mathrm{r}}{x})=M_{h}-s M_{s} \geq 0 \\
& g_{8}(\stackrel{\mathrm{r}}{x})=T \geq 0 \\
& M_{h}=\frac{2}{3} \mu F Z \frac{r_{0}^{3}-r_{i}^{3}}{r_{0}^{2}-r_{i}^{3}} P_{r z}=\frac{F}{\pi\left(r_{0}^{2}-r_{i}^{2}\right)} \\
& v_{s r}=\frac{2 \pi n\left(r_{0}^{3}-r_{i}^{3}\right)}{90\left(r_{0}^{2}-r_{i}^{2}\right)} T=\frac{I_{Z} \pi n}{30\left(M_{h}+M_{f}\right)} \\
& \Delta r=20 \mathrm{~mm}, I_{Z}=55 \mathrm{kgmm}{ }^{2}, P_{\max }=1 \mathrm{MPa}, \\
& F_{\text {max }}=1000 \mathrm{~N}, T_{\text {max }}=15 \mathrm{~s}, \mu=0.5, \\
& s=1.5, M_{s}=40 \mathrm{Nm}, M_{f}=3 \mathrm{Nm}, n=250 \mathrm{rpm}, \\
& v_{s_{\max }}=10 \mathrm{~m} \cdot \mathrm{s}^{-1}, l_{\text {max }}=30 \mathrm{~mm}, \\
& r_{i_{\min }}=60, r_{i_{\max }}=80, r_{0_{\min }}=90, r_{0_{\max }}=110, \\
& t_{\text {min }}=1.5, t_{\max }=3, F_{\min }=600, \\
& F_{\text {max }}=1000, Z_{\text {min }}=2, Z_{\text {max }}=9 .
\end{aligned}
$$

This work compared CPA with $\mathrm{WCA}^{[95]}, \mathrm{PVS}^{[96]}$, and TLBO to minimize the quality of multiple disc clutch brakes. Table 9 shows that the quality of CPA, reaching 0.313656 , is equal to or less than that of other algorithms. This indicates that the proposed algorithm has a stronger optimization ability and can find more high-quality problem solutions.

\section{Conclusions and future work}

This paper proposes a metaheuristic algorithm, named the Colony Predation Algorithm (CPA), inspired by social animals' characteristics to solve the optimization problem. The algorithm achieves the correct balance between exploitation and exploration and can quickly converge in the early and middle stages without falling into Local Optimization (LO). The algorithm 
motivated by the common foraging characteristics of social animals follows the mechanism based on the idea of selective abandonment to simulate the impact of this strategy on individual activities.

This study qualitatively analyzed the algorithm according to the four-index search history, first-dimensional trajectory, average fitness, and convergence curve. The algorithm's performance was evaluated on benchmark and CE2014 functions, and the Friedman test and Wilcoxon test were used for statistical evaluation. The experimental results show that the algorithm has a strong search ability to find the target solution space quicker and subsequently perform exploitation more effectively than other algorithms. The CPA can also identify the optimal solution faster and better on complex multimode functions and exhibits a stronger exploitation ability on complex functions.

Simultaneously, to verify its applicability in practical problems, CPA was applied to tension-compression spring, welded beam, pressure vessel, and other engineering problems. The experimental results show reveal that CPA's average is about 0.9 lower than the second $\mathrm{DE}$ and about 0.84 lower than the second improved DECLS. Thus, the CPA can achieve the optimization of production engineering problems and significantly reduce manufacturing costs.

This work further streamlined the CPA to make it easier to add new mechanisms. In future research, CPA can be applied for parameter optimization, binary feature selection, and image segmentation of machine learning algorithms and combined with machine learning to predict disease for disease prediction.

\section{Acknowledgment}

This research was supported by the National Natural Science Foundation of China (62076185, U1809209). We acknowledge the efforts of Ali Asghar Heidari (https://aliasgharheidari.com) during the preparation and development of this research. We also thank the comments of reviewers and editor.

Open Access This article is licensed under a Creative Commons Attribution 4.0 International License, which permits use, sharing, adaptation, distribution and reproduction in any medium or format, as long as you give appropriate credit to the original author(s) and the source, provide a link to the Creative Commons licence, and indicate if changes were made.

The images or other third party material in this article are included in the article's Creative Commons licence, unless indicated otherwise in a credit line to the material. If material is not included in the article's Creative Commons licence and your intended use is not permitted by statutory regulation or exceeds the permitted use, you will need to obtain permission directly from the copyright holder.

To view a copy of this licence, visit http://creativecommons.org/licenses/by/4.0/.

\section{References}

[1] Huang H, Feng X A, Zhou S Y, Jiang J H, Chen H L, Li Y P, Li C Y. A new fruit fly optimization algorithm enhanced support vector machine for diagnosis of breast cancer based on high-level features. Bmc Bioinformatics, 2019, 20, https://doi.org/10.1186/s12859-019-2771-z.

[2] Cao B, Zhao J W, Gu Y, Ling Y B, Ma X L. Applying graph-based differential grouping for multiobjective large-scale optimization. Swarm and Evolutionary Computation, 2020, 53, 100626.

[3] Fu X W, Pace P, Aloi G, Yang L, Fortino G. Topology optimization against cascading failures on wireless sensor networks using a memetic algorithm. Computer Networks, 2020, 107327.

[4] Cao B, Zhao J W, Yang P, Gu Y, Muhammad K, Rodrigues J J, De Albuquerque V H C. Multiobjective 3-D topology optimization of next-generation wireless data center network. IEEE Transactions on Industrial Informatics, 2019, 16, 3597-3605.

[5] Cao B, Dong W N, Lv Z H, Gu Y, Singh S, Kumar P. Hybrid microgrid many-objective sizing optimization with fuzzy decision. IEEE Transactions on Fuzzy Systems, 2020.

[6] Zeng G Q, Lu Y Z, Mao W J. Modified extremal optimization for the hard maximum satisfiability problem. Journal of Zhejiang University SCIENCE C, 2011, 12, 589-596.

[7] Zeng G Q, Chen J, Dai Y X, Li L M, Zheng C W, Chen M R. Design of fractional order PID controller for automatic regulator voltage system based on multi-objective extremal optimization. Neurocomputing, 2015, 160, 173-184.

[8] Gupta S, Deep K, Heidari A A, Moayedi H, Chen H L. Harmonized salp chain-built optimization. Engineering with Computers, 2019, https://doi.org/10.1007/s00366-019- 
00871-5.

[9] Zhang H L, Cai Z N, Ye X J, Wang M J, Kuang F J, Chen H L, Li C Y, Li Y P. A multi-strategy enhanced salp swarm algorithm for global optimization. Engineering with Computers, 2020, https://doi.org/10.1007/s00366-02001099-4.

[10] Xu Y T, Chen H L, Luo J, Zhang Q, Jiao S, Zhang X Q. Enhanced Moth-flame optimizer with mutation strategy for global optimization. Information Sciences, 2019, 492, 181-203.

[11] Heidari A A, Ali Abbaspour R, Chen H L. Efficient boosted grey wolf optimizers for global search and kernel extreme learning machine training. Applied Soft Computing, 2019, 81, 105521.

[12] Song S M, Wang P J, Heidari A A, Wang M J, Zhao X H, Chen H L, He W M, Xu S L. Dimension decided Harris hawks optimization with Gaussian mutation: Balance analysis and diversity patterns. Knowledge-Based Systems, 2020, https://doi.org/10.1016/j.knosys.2020.106425.

[13] Zhang Y N, Liu R J, Wang X, Chen H L, Li C Y. Boosted binary Harris hawks optimizer and feature selection. Engineering with Computers, 2020. https://doi.org/10.1007/s00366-020-01028-5.

[14] Xu X, Chen H L. Adaptive computational chemotaxis based on field in bacterial foraging optimization. Soft Computing, 2014, 18, 797-807.

[15] Fan Y, Wang P J, Mafarja M, Wang M J, Zhao X H, Chen H L. A bioinformatic variant fruit fly optimizer for tackling optimization problems. Knowledge-Based Systems, 2021, 213, 106704.

[16] Yu H L, Li W S, Chen C C, Liang J, Gui W Y, Wang M J, Chen H L. Dynamic Gaussian bare-bones fruit fly optimizers with abandonment mechanism: Method and analysis. Engineering with Computers, 2020, https://doi.org/10.1007/s00366-020-01174-w.

[17] Wang X Y, Chen H L, Heidari A A, Zhang X, Xu J, Xu Y T, Huang H. Multi-population following behavior-driven fruit fly optimization: A Markov chain convergence proof and comprehensive analysis. Knowledge-Based Systems, 2020, 210, 106437.

[18] Ding L, Li S, Gao H B, Chen C, Deng Z Q. Adaptive partial reinforcement learning neural network-based tracking control for wheeled mobile robotic systems. IEEE Transactions on Systems, Man, and Cybernetics: Systems, 2018, 50, 2512-2523.

[19] Wang J H, Zhu P S, He B T, Deng G Y, Zhang C L, Huang X. An adaptive neural sliding mode control with ESO for uncertain nonlinear systems. International Journal of Control, Automation and Systems, 2020.

[20] Hu J W, Wang M, Zhao C H, Pan Q, Du C. Formation control and collision avoidance for multi-UAV systems based on Voronoi partition. Science China Technological Sciences, 2020. 63, 65-72.

[21] Qian J M, Feng S J, Tao T Y, Hu Y, Li Y X, Chen Q, Zuo C. Deep-learning-enabled geometric constraints and phase unwrapping for single-shot absolute $3 \mathrm{~d}$ shape measurement. APL Photonics, 2020, 5, 046105.

[22] Qian J M, Feng S J, Li Y X, Tao T Y, Han J, Chen Q, Zuo C. Single-shot absolute 3D shape measurement with deep-learning-based color fringe projection profilometry. Optics Letters, 2020, 45, 1842-1845.

[23] Huang Y C, Wang J H, Wang F, He B T. Event-triggered adaptive finite-time tracking control for full state constraints nonlinear systems with parameter uncertainties and given transient performance. ISA Transactions, 2021, 108, 131-143.

[24] Chen Z C, Wang J H, Ma K M, Huang X, Wang T. Fuzzy adaptive two - bits - triggered control for nonlinear uncertain system with input saturation and output constraint. International Journal of Adaptive Control and Signal Processing, 2020, 34, 543-559.

[25] Shida H, Fei G, Quan Z, Ding H. MRMD2.0: A python tool for machine learning with feature ranking and reduction. Current Bioinformatics, 2020, 15, 1213-1221.

[26] Yang S M, Deng B, Wang J, Li H Y, Lu M L, Che Y Q, Wei X L, Loparo K A. Scalable digital neuromorphic architecture for large-scale biophysically meaningful neural network with multi-compartment neurons. IEEE transactions on neural networks and learning systems, 2019, 31, 148-162.

[27] Wang X F, Gao P, Liu Y F, Li H F, Lu F. Predicting thermophilic proteins by machine learning. Current Bioinformatics, 2020, 15, 493-502.

[28] Sun Y, Yen G G, Yi Z. IGD indicator-based evolutionary algorithm for many-objective optimization problems. IEEE Transactions on Evolutionary Computation, 2019, 23, 173-187.

[29] Sun Y, Xue B, Zhang M, Yen G G, Lv J. Automatically designing CNN architectures using the genetic algorithm for image classification. IEEE Transactions on Cybernetics, 2020, 1-15.

[30] Bai B, Guo Z W, Zhou C, Zhang W, Zhang J Y. Application of adaptive reliability importance sampling-based extended domain PSO on single mode failure in reliability engineering. Information Sciences, 2021, 546, 42-59. 
[31] Yang B, Wang J B, Yu L, Shu H C, Yu T, Zhang X S, Yao W, Sun L M. A critical survey on proton exchange membrane fuel cell parameter estimation using meta-heuristic algorithms. Journal of Cleaner Production, 2020, 265, 121660 .

[32] Holland J H. Genetic algorithms. Scientific American, 1992, 267, 66-72.

[33] Storn R, Price K. Differential evolution - A simple and efficient heuristic for global optimization over continuous spaces. Journal of Global Optimization, 1997, 11, 341-359.

[34] Zhao C H, Li J Y. Equilibrium selection under the bayes-based strategy updating rules. Symmetry, 2020, 12, 739.

[35] Koza J R, Rice J P. Automatic programming of robots using genetic programming. Proceedings of the Tenth National Conference on Artificial Intelligence, AAAI Press, San Jose, California, 1992, 194-201.

[36] Hansen N, Ller S D M, Koumoutsakos P. Reducing the time complexity of the derandomized evolution strategy with covariance matrix adaptation (CMA-ES). Evolutionary Computation, 2003, 11, 1-18.

[37] Xin Y, Yong L, Guangming L. Evolutionary programming made faster. IEEE Transactions on Evolutionary Computation, 1999, 3, 82-102.

[38] Kirkpatrick S, Gelatt C D, Vecchi M P. Optimization by simulated annealing. Readings in Computer Vision, Fischler M A, Firschein O, eds., Morgan Kaufmann, San Francisco, USA, 1987, 606-615.

[39] Rashedi E, Nezamabadi-Pour H, Saryazdi S. GSA: A gravitational search algorithm. Information Sciences, 2009, 179, 2232-2248.

[40] Mirjalili S, Mirjalili S M, Lewis A. Grey wolf optimizer. Advances in Engineering Software, 2014, 69, 46-61.

[41] Yang X S. A new metaheuristic bat-inspired algorithm. Nature Inspired Cooperative Strategies for Optimization (NISCO 2010), 2010, 284, 65-74.

[42] Gandomi A H, Yang X S, Alavi A H. Cuckoo search algorithm: A metaheuristic approach to solve structural optimization problems. Engineering with Computers, 2013. 29, 17-35.

[43] Karaboga D, Basturk B. A powerful and efficient algorithm for numerical function optimization: Artificial bee colony (ABC) algorithm. Journal of Global Optimization, 2007, 39, 459-471.

[44] Li S M, Chen H L, Wang M J, Heidari A A, Mirjalili S. Slime mould algorithm: A new method for stochastic optimization. Future Generation Computer Systems, 2020, 111, 300-323.
[45] Heidari A A, Mirjalili S, Faris H, Aljarah I, Mafarja M, Chen H L. Harris hawks optimization: Algorithm and applications. Future Generation Computer Systems-the International Journal of Escience, 2019, 97, 849-872.

[46] Yang Y T, Chen H L, Asghar Heidari A, Gandomi A H. Hunger games search: Visions, conception, implementation, deep analysis, perspectives, and towards performance shifts. Expert Systems with Applications, 2021, https://doi.org/10.1016/j.eswa.2021.114864.

[47] Gandomi A H, Alavi A H. Krill herd: A new bio-inspired optimization algorithm. Communications in Nonlinear Science and Numerical Simulation, 2012, 17, 4831-4845.

[48] Wang G G. Moth search algorithm: A bio-inspired metaheuristic algorithm for global optimization problems. Memetic Computing, 2018, 10, 151-164.

[49] Feng Y H, Deb S, Wang G G, Alavi A H. Monarch butterfly optimization: A comprehensive review. Expert Systems with Applications, 2021, 168, 114418.

[50] Mirjalili S. Moth-flame optimization algorithm: A novel nature-inspired heuristic paradigm. Knowledge-Based Systems, 2015, 89, 228-249.

[51] Faramarzi A, Heidarinejad M, Mirjalili S, Gandomi A H. Marine predators algorithm: A nature-inspired metaheuristic. Expert Systems with Applications, 2020, 152, 113377.

[52] Mirjalili S, Lewis A. The whale optimization algorithm. Advances in Engineering Software, 2016, 95, 51-67.

[53] Glover F, Marti R. Tabu Search, in Metaheuristic Procedures for Training Neutral Networks, Alba E, Martí R, eds., Springer, Boston, USA, 2006, 53-69.

[54] Rao R V, Savsani V J, Vakharia D P, Teaching-learning-based optimization: A novel method for constrained mechanical design optimization problems. Computer-Aided Design, 2011, 43, 303-315.

[55] Gao N S, Luo D D, Cheng B Z, Hou H. Teaching-learning-based optimization of a composite metastructure in the $0-10 \mathrm{kHz}$ broadband sound absorption range. The Journal of the Acoustical Society of America, 2020, 148, EL125-EL129.

[56] Wolpert D H, Macready W G. No free lunch theorems for optimization. IEEE Transactions on Evolutionary Computation, 1997, 1, 67-82.

[57] Anholt B, Werner E. Interaction between food availability and predation mortality mediated by adaptive behavior. Ecology, 1995, 76, 2230-2234.

[58] Cooper S. Optimal hunting group size: The need for lions to defend their kills against loss to spotted hyaenas. African Journal of Ecology, 1991, 29, 130-136. 
[59] Persons M H, Walker S E, Rypstra A L, Marshall S D. Wolf spider predator avoidance tactics and survival in the presence of diet-associated predator cues (Araneae: Lycosidae). Animal Behaviour, 2001, 61, 43-51.

[60] Coleman S L, Brown V R, Levine D S, Mellgren R L. A neural network model of foraging decisions made under predation risk. Cognitive, Affective, \& Behavioral Neuroscience, 2005, 5, 434-451.

[61] Khater M, Murariu D, Gras R. Predation risk tradeoffs in prey: effects on energy and behaviour. Theoretical Ecology, 2016, 9, 251-268.

[62] Shi K B, Wang J, Tang Y Y, Zhong S M. Reliable asynchronous sampled-data filtering of $\mathrm{T}-\mathrm{S}$ fuzzy uncertain delayed neural networks with stochastic switched topologies. Fuzzy Sets and Systems, 2020, 381, 1-25.

[63] Shi K B, Wang J, Zhong S M, Tang Y Y, Cheng J. Non-fragile memory filtering of TS fuzzy delayed neural networks based on switched fuzzy sampled-data control. Fuzzy Sets and Systems, 2020, 394, 40-64.

[64] Shi K B, Tang Y Y, Zhong S M, Yin C, Huang X G, Wang W Q. Nonfragile asynchronous control for uncertain chaotic Lurie network systems with Bernoulli stochastic process. International Journal of Robust and Nonlinear Control, 2018, 28, 1693-1714.

[65] Ni T, Chang H, Song T, Xu Q, Huang Z, Liang H, Yan A, Wen $X$. Non-intrusive online distributed pulse shrinking-based interconnect testing in 2.5D IC. IEEE Transactions on Circuits and Systems II: Express Briefs, 2020, 67, 2657-2661.

[66] Zhang H, Qiu Z, Cao J, Abdel-Aty M, Xiong L. Event-triggered ysnchronization for neutral-type semi-markovian neural networks with partial mode-dependent time-varying delays. IEEE Transactions on Neural Networks and Learning Systems, 2020, 31, $4437-4450$

[67] Hu J, Chen H L, Heidari A A, Wang M J, Zhang X Q, Chen Y, Pan Z F. Orthogonal learning covariance matrix for defects of grey wolf optimizer: Insights, balance, diversity, and feature selection. Knowledge-Based Systems, 2021, 213, 106684.

[68] Shan W F, Qiao Z L, Heidari A A, Chen H L, Turabieh H, Teng Y T. Double adaptive weights for stabilization of moth flame optimizer: Balance analysis, engineering cases, and medical diagnosis. Knowledge-Based Systems, 2020, 106728 .

[69] Tu J Z, Chen H L, Liu J C, Heidari A A, Zhang X Q, Wang M J, Ruby R, Pham Q-V. Evolutionary biogeography-based whale optimization methods with communication structure: Towards measuring the balance. Knowledge-Based Systems, 2021, 212, 106642.

[70] Zhao D, Liu L, Yu F H, Heidari A A, Wang M J, Liang G X, Muhammad K, Chen H. Chaotic random spare ant colony optimization for multi-threshold image segmentation of 2D Kapur entropy. Knowledge-Based Systems, 2020, https://doi.org/10.1016/j.knosys.2020.106510.

[71] Zhang Y N, Liu R J, Heidari A A, Wang X, Chen Y, Wang M J, Chen H L. Towards augmented kernel extreme learning models for bankruptcy prediction: Algorithmic behavior and comprehensive analysis. Neurocomputing, 2020.

[72] Chen H, Heidari A A, Chen H L, Wang M J, Pan Z F, Gandomi A H. Multi-population differential evolution-assisted Harris hawks optimization: Framework and case studies. Future Generation Computer Systems, 2020, 111, 175-198.

[73] Mirjalili S. SCA: A sine cosine algorithm for solving optimization problems. Knowledge-Based Systems, 2016, 96, $120-133$.

[74] Mirjalili S, Gandomi A H, Mirjalili S Z, Saremi S, Faris H, Mirjalili S M. Salp swarm algorithm: A bio-inspired optimizer for engineering design problems. Advances in Engineering Software, 2017, 114, 163-191.

[75] Kennedy J. Particle Swarm Optimization, in Encyclopedia of Machine Learning, Sammut C, Webb G I, eds., Springer, Boston, USA, 2010, 760-766.

[76] Yang X S. Firefly Algorithms for multimodal optimization. Stochastic Algorithms: Foundations and Applications, Springer Berlin Heidelberg, Berlin, Heidelberg, Germany, 2009.

[77] Gupta S, Deep K. A hybrid self-adaptive sine cosine algorithm with opposition based learning. Expert Systems with Applications, 2019, 119, 210-230.

[78] Abd Elaziz M, Oliva D, Xiong S. An improved opposition-based sine cosine algorithm for global optimization. Expert Systems with Applications, 2017, 90, 484-500.

[79] Sun Y, Wang X, Chen Y, Liu Z. A modified whale optimization algorithm for large-scale global optimization problems. Expert Systems with Applications, 2018, 114, 563-577.

[80] Tubishat M, Abushariah M A M, Idris N, Aljarah I. Improved whale optimization algorithm for feature selection in Arabic sentiment analysis. Applied Intelligence, 2018.

[81] Yong J, He F, Li H, Zhou W. A novel bat algorithm based on collaborative and dynamic learning of opposite population. 
IEEE 22nd International Conference on Computer Supported Cooperative Work in Design (CSCWD), 2018.

[82] Adarsh B R, Raghunathan T, Jayabarathi T, Yang X S, Economic dispatch using chaotic bat algorithm. Energy, 2016, 96, 666-675.

[83] Jia D L, Zheng G X, Qu B Y, Khan M K. A hybrid particle swarm optimization algorithm for high-dimensional problems. Computers \& Industrial Engineering, 2011, 61, $1117-1122$.

[84] Chen W, Zhang J, Lin Y, Chen N, Zhan Z, Chung H S, Li Y, Shi Y. Particle swarm optimization with an aging leader and challengers. IEEE Transactions on Evolutionary Computation, 2013, 17, 241-258.

[85] Jia D L, Zheng G X, Khurram Khan M. An effective memetic differential evolution algorithm based on chaotic local search. Information Sciences, 2011, 181, 3175-3187.

[86] Kaveh A, Khayatazad M. A new meta-heuristic method: Ray optimization. Computers \& Structures, 2012, 112-113, 283-294.

[87] Mahdavi M, Fesanghary M, Damangir E. An improved harmony search algorithm for solving optimization problems. Applied Mathematics \& Computation, 2007, 188, 1567-1579.

[88] Huang F Z, Wang L, He Q. An effective co-evolutionary differential evolution for constrained optimization. Applied Mathematics and Computation, 2007, 186, 340-356.

[89] He Q, Wang L. An effective co-evolutionary particle swarm optimization for constrained engineering design problems. Engineering Applications of Artificial Intelligence, 2007, 20, 89-99.

[90] He Q, Wang L. A hybrid particle swarm optimization with a feasibility-based rule for constrained optimization. Applied Mathematics and Computation, 2007, 186, 1407-1422.

[91] Kannan B K, Kramer S N. An augmented lagrange multiplier based method for mixed integer discrete continuous optimization and its applications to mechanical design. Journal of Mechanical Design, 1994, 116, 405-411.

[92] Sandgren E. Nonlinear integer and discrete programming in mechanical design optimization. Journal of Mechanical Design, 1990, 112, 223-229.

[93] Wang G G. Adaptive response surface method using inherited Latin hypercube design points. Journal of Mechanical Design, 2003, 125, 210-220.

[94] Cheng M Y, Prayogo D. Symbiotic organisms search: A new metaheuristic optimization algorithm. Computers \& Structures, 2014, 139, 98-112.

[95] Eskandar H, Sadollah A, Bahreininejad A, Hamdi M. Water cycle algorithm - A novel metaheuristic optimization method for solving constrained engineering optimization problems. Computers \& Structures, 2012, 110-111, 151-166.

[96] Savsani P, Savsani V. Passing vehicle search (PVS): A novel metaheuristic algorithm. Applied Mathematical Modelling, 2016, 40, 3951-3978.

\section{Appendix A}

Table A1 Description of the 23 benchmark functions

\begin{tabular}{ccccc}
\hline Function & Function equation & Dim & Range & $f_{\min }$ \\
\hline F1 & $f_{1}(x)=\sum_{i=1}^{n} x^{2}$ & 30 & {$[-100,100]$} & 0 \\
F2 & $f_{2}(x)=\sum_{i=1}^{n}\left|x_{i}\right|+\prod_{i=1}^{n}\left|x_{i}\right|$ & 30 & {$[-10,10]$} & 0 \\
F3 & $f_{3}(x)=\sum_{i=1}^{n}\left(\sum_{j-1}^{i} x_{j}\right)^{2}$ & 30 & {$[-100,100]$} & 0 \\
F4 & $f_{4}(x)=\max _{i}\left\{\left|x_{i}\right|, 1 \leq i \leq n\right\}$ & 30 & {$[-100,100]$} & 0 \\
F5 & $f_{5}(x)=\sum_{i=1}^{n-1}\left[100\left(x_{i+1}-x_{i}^{2}\right)^{2}+\left(x_{i}-1\right)^{2}\right]$ & 30 & {$[-30,30]$} & 0 \\
F6 & $f_{6}(x)=\sum_{i=1}^{n}\left(\left[x_{i}+0.5\right]\right)^{2}$ & 30 & {$[-100,100]$} & 0 \\
F7 & $f_{7}(x)=\sum_{i=1}^{n} i x^{4}+\operatorname{random}[0,1)$ & 30 & {$[-1.28,1.28]$} & 0 \\
F8 & $f_{8}(x)=\sum_{i=1}^{n}-x \sin \left(\sqrt{\left|x_{i}\right|}\right)$ & 30 & {$[-500,500]$} & -418.982 \\
F9 & $f_{9}(x)=\sum_{i=1}^{n}\left[x_{i}^{2}-10 \cos \left(2 \pi x_{i}\right)+10\right]$ & 30 & {$[-5.12,5.12]$} & 0 \\
F10 & $f_{10}(x)=-20 e^{-0.2 \cdot \sqrt{\frac{1}{\pi}} \sum_{i=1}^{n} x_{i}}-e^{\frac{1}{2} \sum_{i=1}^{n} \cos \left(2 \pi x_{i}\right)}+20+e$ & 30 & {$[-32,32]$} & 0 \\
\hline
\end{tabular}




\begin{tabular}{|c|c|c|c|c|}
\hline F11 & $f_{11}(x)=\frac{1}{4000} \sum_{i=1}^{n} x_{i}^{2}-\prod_{i=1}^{n} \cos \left(\frac{x_{i}}{\sqrt{i}}\right)+1$ & 30 & {$[-600,600]$} & 0 \\
\hline F12 & $\begin{aligned} f_{12}(x)= & \frac{\pi}{n}\left\{10 \sin \left(a y_{1}\right)+\sum_{i=1}^{n=1}\left(y_{i}-1\right)^{2}\left[1+\sin ^{2}\left(\pi y_{i+1}\right)\right]+\right. \\
& \left.\left(y_{n}-1\right)^{2}+\sum_{i=1}^{n} \mu\left(x_{i}, 10,100,4\right)\right\}\end{aligned}$ & 30 & {$[-50,50]$} & 0 \\
\hline F13 & $\begin{array}{l}f_{13}(x)=0.1\left\{\sin ^{2}\left(3 \pi x_{i}\right)+\sum_{i=1}^{n}\left(x_{i}-1\right)^{2}\left[1+\sin ^{2}\left(3 \pi x_{i}+1\right)\right]+\right. \\
\left.\left(x_{n}-1\right)^{2}\left[1+\sin ^{2}\left(2 \pi x_{n}\right)\right]+\sum_{i=1}^{n} \mu\left(x_{i}, 5,100,4\right)\right\}\end{array}$ & 30 & {$[-50,50]$} & 0 \\
\hline F14 & $f_{14}(x)=\left(\frac{1}{5000}+\sum_{j=1}^{25} \frac{1}{j+\sum_{i=1}^{2}\left(x_{i}+a_{i j}\right)^{6}}\right)^{-1}$ & 2 & {$[-65,65]$} & 1 \\
\hline F15 & $f_{15}(x)=\sum_{i=1}^{11}\left[a_{i}-\frac{x_{1}\left(b_{i}^{2}+b_{i} x_{2}\right)}{b_{i}^{2}+b_{i} x_{3}+x_{4}}\right]^{2}$ & 4 & {$[-5,5]$} & 0.00030 \\
\hline F16 & $f_{16}(x)=4 x_{1}^{2}-2.1 x_{1}^{4}+\frac{1}{3} x_{1}^{6}+x_{1} x_{2}-4 x_{2}^{2}+4 x_{2}^{4}$ & 2 & {$[-5,5]$} & -1.0316 \\
\hline F17 & $f_{17}(x)=\left(x_{2}-\frac{5.1}{4 \pi^{2}} x_{1}^{2}+\frac{5}{\pi} x_{1}-6\right)^{2}+10\left(1-\frac{1}{8 \pi} \cos \left(x_{1}\right)+10\right)$ & 2 & {$[-5,5]$} & 0.398 \\
\hline F18 & $\begin{array}{l}f_{18}(x)=\left[1+\left(x_{1}+x_{2}+1\right)^{2} \times\left(19-14 x_{1}+3 x_{1}^{2}-14 x_{2}+6 x_{1} x_{2}+3 x_{2}^{2}\right)\right] \times \\
{\left[30+\left(2 x_{1}-3 x_{2}\right)^{2} \times\left(18-32 x_{1}+12 x_{1}^{2}+48 x_{2}-36 x_{1} x_{2}+27 x_{2}^{2}\right)\right]}\end{array}$ & 2 & {$[-2,2]$} & 3 \\
\hline F19 & $f_{19}(x)=-\sum_{i=1}^{4} c_{i} e^{-\sum_{j=1}^{3} a_{j i}\left(x_{j}-p_{i j}\right)^{2}}$ & 3 & {$[1,3]$} & -3.86 \\
\hline F20 & $f_{20}(x)=-\sum_{i=1}^{4} c_{i} e^{-\sum_{j=1}^{6} a_{i j}\left(x_{j}-p_{i j}\right)^{2}}$ & 6 & {$[0,1]$} & -3.32 \\
\hline F21 & $f_{21}(x)=-\sum_{i=1}^{5}\left[\left(X-a_{i}\right)\left(X-a_{i}\right)^{T}+c_{i}\right]^{-1}$ & 4 & {$[0,10]$} & -10.1532 \\
\hline F22 & $f_{22}(x)=-\sum_{i=1}^{7}\left[\left(X-a_{i}\right)\left(X-a_{i}\right)^{T}+c_{i}\right]^{-1}$ & 4 & {$[0,10]$} & -10.4028 \\
\hline F23 & $f_{23}(x)=-\sum_{i=1}^{10}\left[\left(X-a_{i}\right)\left(X-a_{i}\right)^{T}+c_{i}\right]^{-1}$ & 4 & {$[0,10]$} & -10.5363 \\
\hline
\end{tabular}

Table A2 Description of IEEE CEC2014 functions

\begin{tabular}{|c|c|c|c|c|}
\hline ID & Function equation & Dim & Range & $f_{\min }$ \\
\hline \multicolumn{5}{|c|}{ Unimodal functions } \\
\hline F24 & Rotated High Conditioned Elliptic Function & 30 & {$[-100,100]$} & 100 \\
\hline F25 & Rotated Bent Cigar Function & 30 & {$[-100,100]$} & 200 \\
\hline F26 & Rotated Discus Function & 30 & {$[-100,100]$} & 300 \\
\hline \multicolumn{5}{|c|}{ Simple multimodal functions } \\
\hline F27 & Shifted and Rotated Rosenbrock's Function & 30 & {$[-100,100]$} & 400 \\
\hline F28 & Shifted and Rotated Ackley’s Function & 30 & {$[-100,100]$} & 500 \\
\hline F29 & Shifted and Rotated Weierstrass Function & 30 & {$[-100,100]$} & 600 \\
\hline F30 & Shifted and Rotated Griewank's Function & 30 & {$[-100,100]$} & 700 \\
\hline F31 & Shifted Restringing's Function & 30 & {$[-100,100]$} & 800 \\
\hline F32 & Shifted and Rotated Restringing's Function & 30 & {$[-100,100]$} & 900 \\
\hline F33 & Shifted Schwefel's Function & 30 & {$[-100,100]$} & 1000 \\
\hline F34 & Shifted and Rotated Schwefel's Function & 30 & {$[-100,100]$} & 1100 \\
\hline F35 & Shifted and Rotated Katsuura Function & 30 & {$[-100,100]$} & 1200 \\
\hline F36 & Shifted and Rotated HappyCat Function & 30 & {$[-100,100]$} & 1300 \\
\hline F37 & Shifted and Rotated HGBat Function & 30 & {$[-100,100]$} & 1400 \\
\hline F38 & Shifted and Rotated Expanded Griewank's plus Rosenbrock's Function & 30 & {$[-100,100]$} & 1500 \\
\hline F39 & Shifted and Rotated Expanded Scoffer's F6 Function & 30 & {$[-100,100]$} & 1600 \\
\hline \multicolumn{5}{|c|}{ Hybrid functions } \\
\hline F40 & Hybrid Function $1(N=3)$ & 30 & {$[-100,100]$} & 1700 \\
\hline F41 & Hybrid Function $2(N=3)$ & 30 & {$[-100,100]$} & 1800 \\
\hline F42 & Hybrid Function $3(N=4)$ & 30 & {$[-100,100]$} & 1900 \\
\hline F43 & Hybrid Function $4(N=4)$ & 30 & {$[-100,100]$} & 2000 \\
\hline F44 & Hybrid Function $5(N=5)$ & 30 & {$[-100,100]$} & 2100 \\
\hline F45 & Hybrid Function $6(N=5)$ & 30 & {$[-100,100]$} & 2200 \\
\hline \multicolumn{5}{|c|}{ Composition functions } \\
\hline F46 & Composition Function $1(N=5)$ & 30 & {$[-100,100]$} & 2300 \\
\hline F47 & Composition Function $2(N=3)$ & 30 & {$[-100,100]$} & 2400 \\
\hline F48 & Composition Function $3(N=3)$ & 30 & {$[-100,100]$} & 2500 \\
\hline F49 & Composition Function $4(N=5)$ & 30 & {$[-100,100]$} & 2600 \\
\hline F50 & Composition Function $5(N=5)$ & 30 & {$[-100,100]$} & 2700 \\
\hline F51 & Composition Function $6(N=5)$ & 30 & {$[-100,100]$} & 2800 \\
\hline F52 & Composition Function $7(N=3)$ & 30 & {$[-100,100]$} & 2900 \\
\hline F53 & Composition Function $8(N=3)$ & 30 & {$[-100,100]$} & 3000 \\
\hline
\end{tabular}


Table A3 Parameter settings of counterparts

\begin{tabular}{|c|c|c|}
\hline Algorithm & Parameter settings & Parametric note \\
\hline SCA & $A=2$ & $A$ is a constant \\
\hline SSA & $c_{1} \in[2,0] ; c_{2} \in[0,1] ; c_{3} \in[0,1]$ & $\begin{array}{c}c_{1} \text { is an exponential decreasing variable; } c_{2} \\
\text { and } c_{3} \text { are random numbers }\end{array}$ \\
\hline GWO & $a=[2,0]$ & $a$ is a linear decreasing variable \\
\hline MFO & $b=1 ; t=[-1,1] ; a \in[-1,-2]$ & $\begin{array}{c}b \text { is a constant; } t \text { is a random number; } a \text { is a } \\
\text { linear decreasing variable; }\end{array}$ \\
\hline WOA & $a_{1}=[2,0] ; a_{2}=[-2,-1] ; b=1$ & $\begin{array}{c}a_{1} \text { and } a_{2} \text { are linear decreasing variables; } b \text { is } \\
\text { a constant }\end{array}$ \\
\hline $\mathrm{ABC}$ & limit $=300$ & limit is a constant \\
\hline GSA & Rpower $=1 ;$ ElitistCheck $=1 ;$ Rnorm $=2$ & $\begin{array}{l}\text { Rpower ElitistCheck and Rnorm are con- } \\
\text { stants }\end{array}$ \\
\hline FA & $a=0.5 ; \beta=0.2 ; \gamma=1$ & $\begin{array}{c}\alpha \text { and } \beta \text { are constants; } \gamma \text { means absorption } \\
\text { coefficient }\end{array}$ \\
\hline PSO & $c_{1}=2 ; c_{2}=2 ; v \operatorname{Max}=6$ & $c_{1}, c_{2}$ and $v M a x$ are constants \\
\hline $\mathrm{DE}$ & beta $_{\min }=0.2 ;$ beta $_{\max }=0.8 ; p C R=0.2$ & $\begin{array}{c}\text { beta means scaling factor; } p C R \text { means } \\
\text { crossover probability }\end{array}$ \\
\hline BA & $A=[1,2] ; r=[0,1] ; Q_{\min }=0 ; Q_{\max }=2$ & $\begin{array}{c}A \text { means loudness; } r \text { means pulse rate; } Q \\
\text { means Frequency }\end{array}$ \\
\hline
\end{tabular}

Table A4 $p$-value of Wilcoxon test obtained from comparison with traditional algorithms on 53 functions

\begin{tabular}{|c|c|c|c|c|c|c|c|c|}
\hline Function & PSO & $\mathrm{DE}$ & $\mathrm{ABC}$ & SCA & SSA & BA & FA & MFO \\
\hline $\mathrm{F} 1$ & $1.734 \mathrm{E}-06$ & $1.734 \mathrm{E}-06$ & $1.734 \mathrm{E}-06$ & $1.734 \mathrm{E}-06$ & $1.734 \mathrm{E}-06$ & $1.734 \mathrm{E}-06$ & $1.734 \mathrm{E}-06$ & $1.697 \mathrm{E}-06$ \\
\hline $\mathrm{F} 2$ & $1.734 \mathrm{E}-06$ & $1.734 \mathrm{E}-06$ & $1.734 \mathrm{E}-06$ & $1.734 \mathrm{E}-06$ & $1.734 \mathrm{E}-06$ & $1.734 \mathrm{E}-06$ & $1.734 \mathrm{E}-06$ & $1.601 \mathrm{E}-06$ \\
\hline F3 & $1.734 \mathrm{E}-06$ & $1.734 \mathrm{E}-06$ & $1.734 \mathrm{E}-06$ & $1.734 \mathrm{E}-06$ & $1.734 \mathrm{E}-06$ & $1.734 \mathrm{E}-06$ & $1.734 \mathrm{E}-06$ & $1.691 \mathrm{E}-06$ \\
\hline $\mathrm{F} 4$ & $1.734 \mathrm{E}-06$ & $1.734 \mathrm{E}-06$ & $1.734 \mathrm{E}-06$ & $1.734 \mathrm{E}-06$ & $1.734 \mathrm{E}-06$ & $1.734 \mathrm{E}-06$ & $1.734 \mathrm{E}-06$ & $1.734 \mathrm{E}-06$ \\
\hline F5 & $1.734 \mathrm{E}-06$ & $2.105 \mathrm{E}-03$ & $1.734 \mathrm{E}-06$ & $1.734 \mathrm{E}-06$ & $4.114 \mathrm{E}-03$ & $1.921 \mathrm{E}-06$ & $1.734 \mathrm{E}-06$ & $4.860 \mathrm{E}-05$ \\
\hline F6 & $1.734 \mathrm{E}-06$ & $1.734 \mathrm{E}-06$ & $1.734 \mathrm{E}-06$ & $1.734 \mathrm{E}-06$ & $1.734 \mathrm{E}-06$ & $1.734 \mathrm{E}-06$ & $1.734 \mathrm{E}-06$ & $4.165 E-01$ \\
\hline F7 & $1.734 \mathrm{E}-06$ & $1.734 \mathrm{E}-06$ & $1.734 \mathrm{E}-06$ & $1.734 \mathrm{E}-06$ & $1.734 \mathrm{E}-06$ & $1.734 \mathrm{E}-06$ & $1.734 \mathrm{E}-06$ & $1.734 \mathrm{E}-06$ \\
\hline F8 & $1.734 \mathrm{E}-06$ & $3.374 \mathrm{E}-04$ & $9.766 \mathrm{E}-04$ & $1.734 \mathrm{E}-06$ & $1.734 \mathrm{E}-06$ & $1.734 \mathrm{E}-06$ & $1.734 \mathrm{E}-06$ & $1.734 \mathrm{E}-06$ \\
\hline F9 & $1.734 \mathrm{E}-06$ & $1.250 \mathrm{E}-01$ & $1.000 \mathrm{E}+00$ & $1.000 \mathrm{E}+00$ & $1.734 \mathrm{E}-06$ & $1.734 \mathrm{E}-06$ & $1.734 \mathrm{E}-06$ & $1.734 \mathrm{E}-06$ \\
\hline F10 & $1.734 \mathrm{E}-06$ & $1.962 \mathrm{E}-07$ & $6.634 \mathrm{E}-07$ & $1.730 \mathrm{E}-06$ & $1.734 \mathrm{E}-06$ & $1.734 \mathrm{E}-06$ & $1.734 \mathrm{E}-06$ & $1.673 \mathrm{E}-06$ \\
\hline F11 & $1.734 \mathrm{E}-06$ & $1.000 E+00$ & $2.189 \mathrm{E}-06$ & $1.000 E+00$ & $1.734 \mathrm{E}-06$ & $1.734 \mathrm{E}-06$ & $1.734 \mathrm{E}-06$ & $2.696 \mathrm{E}-05$ \\
\hline F12 & $1.734 \mathrm{E}-06$ & $1.734 \mathrm{E}-06$ & $1.734 \mathrm{E}-06$ & $1.734 \mathrm{E}-06$ & $1.734 \mathrm{E}-06$ & $1.734 \mathrm{E}-06$ & $1.734 \mathrm{E}-06$ & $1.478 \mathrm{E}-05$ \\
\hline F13 & $1.734 \mathrm{E}-06$ & $1.734 \mathrm{E}-06$ & $1.734 \mathrm{E}-06$ & $1.734 \mathrm{E}-06$ & $1.734 \mathrm{E}-06$ & $1.734 \mathrm{E}-06$ & $1.734 \mathrm{E}-06$ & $4.178 \mathrm{E}-04$ \\
\hline F14 & $1.734 \mathrm{E}-06$ & $1.000 \mathrm{E}+00$ & $1.000 \mathrm{E}+00$ & $1.734 \mathrm{E}-06$ & $1.000 \mathrm{E}+00$ & $1.734 \mathrm{E}-06$ & $1.734 \mathrm{E}-06$ & $4.883 \mathrm{E}-04$ \\
\hline F15 & $1.734 \mathrm{E}-06$ & $5.706 \mathrm{E}-02$ & $1.734 \mathrm{E}-06$ & $1.734 \mathrm{E}-06$ & $2.879 \mathrm{E}-06$ & $1.734 \mathrm{E}-06$ & $1.734 \mathrm{E}-06$ & $5.302 \mathrm{E}-05$ \\
\hline F16 & $1.734 \mathrm{E}-06$ & $1.000 \mathrm{E}+00$ & $1.000 E+00$ & $1.734 \mathrm{E}-06$ & $3.906 \mathrm{E}-03$ & $1.734 \mathrm{E}-06$ & $1.734 \mathrm{E}-06$ & $1.000 \mathrm{E}+00$ \\
\hline F17 & $1.734 \mathrm{E}-06$ & $1.000 \mathrm{E}+00$ & $1.000 E+00$ & $1.734 \mathrm{E}-06$ & $1.000 \mathrm{E}+00$ & $1.734 \mathrm{E}-06$ & $1.734 \mathrm{E}-06$ & $1.000 \mathrm{E}+00$ \\
\hline F18 & $1.734 \mathrm{E}-06$ & $3.906 \mathrm{E}-02$ & $5.791 \mathrm{E}-04$ & $1.734 \mathrm{E}-06$ & $9.987 \mathrm{E}-07$ & $1.734 \mathrm{E}-06$ & $1.734 \mathrm{E}-06$ & $5.737 E-02$ \\
\hline F19 & $1.734 \mathrm{E}-06$ & $1.000 \mathrm{E}+00$ & $1.000 E+00$ & $1.734 \mathrm{E}-06$ & $6.250 \mathrm{E}-02$ & $1.734 \mathrm{E}-06$ & $1.734 \mathrm{E}-06$ & $1.000 \mathrm{E}+00$ \\
\hline F20 & $1.734 \mathrm{E}-06$ & $5.000 \mathrm{E}-01$ & $1.000 \mathrm{E}+00$ & $1.734 \mathrm{E}-06$ & $1.732 \mathrm{E}-06$ & $1.734 \mathrm{E}-06$ & $1.734 \mathrm{E}-06$ & $6.648 \mathrm{E}-06$ \\
\hline $\mathrm{F} 21$ & $7.731 \mathrm{E}-03$ & $1.563 \mathrm{E}-02$ & $1.563 \mathrm{E}-02$ & $1.734 \mathrm{E}-06$ & $3.709 \mathrm{E}-01$ & $1.414 \mathrm{E}-01$ & $3.709 E-01$ & $1.451 \mathrm{E}-04$ \\
\hline F22 & $8.936 E-01$ & $4.883 \mathrm{E}-04$ & $4.883 \mathrm{E}-04$ & $4.286 \mathrm{E}-06$ & $2.210 \mathrm{E}-01$ & $4.908 \mathrm{E}-01$ & $2.452 \mathrm{E}-01$ & $6.837 \mathrm{E}-01$ \\
\hline F23 & $8.290 \mathrm{E}-01$ & $4.883 \mathrm{E}-04$ & $4.883 \mathrm{E}-04$ & $4.897 \mathrm{E}-04$ & $2.059 \mathrm{E}-01$ & $6.583 E-01$ & $2.623 E-01$ & $4.403 \mathrm{E}-01$ \\
\hline F24 & $1.734 \mathrm{E}-06$ & $1.734 \mathrm{E}-06$ & $1.734 \mathrm{E}-06$ & $1.734 \mathrm{E}-06$ & $4.729 \mathrm{E}-06$ & $9.711 \mathrm{E}-05$ & $1.734 \mathrm{E}-06$ & $1.734 \mathrm{E}-06$ \\
\hline $\mathrm{F} 25$ & $1.734 \mathrm{E}-06$ & $3.882 \mathrm{E}-06$ & $3.515 \mathrm{E}-06$ & $1.734 \mathrm{E}-06$ & $7.813 E-01$ & $1.734 \mathrm{E}-06$ & $1.734 \mathrm{E}-06$ & $1.734 \mathrm{E}-06$ \\
\hline F26 & $1.734 \mathrm{E}-06$ & $3.854 \mathrm{E}-03$ & $2.879 \mathrm{E}-06$ & $1.734 \mathrm{E}-06$ & $2.304 \mathrm{E}-02$ & $3.882 \mathrm{E}-06$ & $1.734 \mathrm{E}-06$ & $1.734 \mathrm{E}-06$ \\
\hline $\mathrm{F} 27$ & $2.623 \mathrm{E}-01$ & $6.035 \mathrm{E}-03$ & $2.255 \mathrm{E}-03$ & $1.734 \mathrm{E}-06$ & $6.564 \mathrm{E}-02$ & $2.304 \mathrm{E}-02$ & $1.734 \mathrm{E}-06$ & $1.734 \mathrm{E}-06$ \\
\hline F28 & $1.734 \mathrm{E}-06$ & $1.734 \mathrm{E}-06$ & $1.734 \mathrm{E}-06$ & $1.734 \mathrm{E}-06$ & $1.734 \mathrm{E}-06$ & $1.734 \mathrm{E}-06$ & $1.734 \mathrm{E}-06$ & $2.353 \mathrm{E}-06$ \\
\hline F29 & $6.984 \mathrm{E}-06$ & $4.048 \mathrm{E}-01$ & $5.706 \mathrm{E}-04$ & $1.734 \mathrm{E}-06$ & $6.435 E-01$ & $1.734 \mathrm{E}-06$ & $1.734 \mathrm{E}-06$ & $3.182 \mathrm{E}-06$ \\
\hline F30 & $1.734 \mathrm{E}-06$ & $6.708 \mathrm{E}-06$ & $4.416 \mathrm{E}-04$ & $1.734 \mathrm{E}-06$ & $1.779 \mathrm{E}-01$ & $1.734 \mathrm{E}-06$ & $1.734 \mathrm{E}-06$ & $1.734 \mathrm{E}-06$ \\
\hline
\end{tabular}




\begin{tabular}{|c|c|c|c|c|c|c|c|c|}
\hline F31 & $1.734 \mathrm{E}-06$ & $2.232 \mathrm{E}-03$ & $3.738 \mathrm{E}-05$ & $1.734 \mathrm{E}-06$ & $1.734 \mathrm{E}-06$ & $1.734 \mathrm{E}-06$ & $1.734 \mathrm{E}-06$ & $1.734 \mathrm{E}-06$ \\
\hline F32 & $1.734 \mathrm{E}-06$ & $3.162 \mathrm{E}-03$ & $2.603 \mathrm{E}-06$ & $1.734 \mathrm{E}-06$ & $3.086 \mathrm{E}-01$ & $1.734 \mathrm{E}-06$ & $1.734 \mathrm{E}-06$ & $1.921 \mathrm{E}-06$ \\
\hline F33 & $1.734 \mathrm{E}-06$ & $1.734 \mathrm{E}-06$ & $8.919 \mathrm{E}-05$ & $1.734 \mathrm{E}-06$ & $1.734 \mathrm{E}-06$ & $1.734 \mathrm{E}-06$ & $1.734 \mathrm{E}-06$ & $1.734 \mathrm{E}-06$ \\
\hline F34 & $1.734 \mathrm{E}-06$ & $1.734 \mathrm{E}-06$ & $4.072 \mathrm{E}-05$ & $1.734 \mathrm{E}-06$ & $3.405 \mathrm{E}-05$ & $1.734 \mathrm{E}-06$ & $1.734 \mathrm{E}-06$ & $2.353 \mathrm{E}-06$ \\
\hline F35 & $1.734 \mathrm{E}-06$ & $1.734 \mathrm{E}-06$ & $4.729 \mathrm{E}-06$ & $1.734 \mathrm{E}-06$ & $6.339 \mathrm{E}-06$ & $1.734 \mathrm{E}-06$ & $1.734 \mathrm{E}-06$ & $1.734 \mathrm{E}-06$ \\
\hline F36 & $3.065 \mathrm{E}-04$ & $5.752 \mathrm{E}-06$ & $1.734 \mathrm{E}-06$ & $1.734 \mathrm{E}-06$ & $3.493 \mathrm{E}-01$ & $6.583 E-01$ & $1.734 \mathrm{E}-06$ & $2.353 \mathrm{E}-06$ \\
\hline F37 & $6.035 \mathrm{E}-03$ & $4.048 \mathrm{E}-01$ & $1.734 \mathrm{E}-06$ & $1.734 \mathrm{E}-06$ & $3.501 \mathrm{E}-02$ & $1.064 E-01$ & $1.734 \mathrm{E}-06$ & $1.734 \mathrm{E}-06$ \\
\hline F38 & $7.712 \mathrm{E}-04$ & $3.389 \mathrm{E}-01$ & $1.287 \mathrm{E}-03$ & $1.734 \mathrm{E}-06$ & $2.957 \mathrm{E}-03$ & $3.182 \mathrm{E}-06$ & $1.734 \mathrm{E}-06$ & $1.734 \mathrm{E}-06$ \\
\hline F39 & $1.734 \mathrm{E}-06$ & $1.734 \mathrm{E}-06$ & $2.536 \mathrm{E}-01$ & $1.734 \mathrm{E}-06$ & $4.729 \mathrm{E}-06$ & $1.734 \mathrm{E}-06$ & $1.734 \mathrm{E}-06$ & $1.921 \mathrm{E}-06$ \\
\hline F40 & $1.734 \mathrm{E}-06$ & $1.734 \mathrm{E}-06$ & $1.734 \mathrm{E}-06$ & $1.734 \mathrm{E}-06$ & $2.067 \mathrm{E}-02$ & $6.035 \mathrm{E}-03$ & $1.734 \mathrm{E}-06$ & $7.691 \mathrm{E}-06$ \\
\hline F41 & $1.734 \mathrm{E}-06$ & $2.585 \mathrm{E}-03$ & $4.534 \mathrm{E}-04$ & $1.734 \mathrm{E}-06$ & $8.590 \mathrm{E}-02$ & $1.734 \mathrm{E}-06$ & $1.734 \mathrm{E}-06$ & $1.359 \mathrm{E}-04$ \\
\hline F42 & $2.843 \mathrm{E}-05$ & $1.714 \mathrm{E}-01$ & $6.320 \mathrm{E}-05$ & $1.734 \mathrm{E}-06$ & $3.405 \mathrm{E}-05$ & $2.597 \mathrm{E}-05$ & $1.734 \mathrm{E}-06$ & $1.734 \mathrm{E}-06$ \\
\hline F43 & $1.734 \mathrm{E}-06$ & $1.734 \mathrm{E}-06$ & $1.734 \mathrm{E}-06$ & $1.734 \mathrm{E}-06$ & $1.734 \mathrm{E}-06$ & $1.734 \mathrm{E}-06$ & $1.734 \mathrm{E}-06$ & $1.734 \mathrm{E}-06$ \\
\hline F44 & $6.639 \mathrm{E}-04$ & $1.734 \mathrm{E}-06$ & $3.515 \mathrm{E}-06$ & $1.734 \mathrm{E}-06$ & $1.714 \mathrm{E}-01$ & $5.577 \mathrm{E}-01$ & $1.734 \mathrm{E}-06$ & $3.882 \mathrm{E}-06$ \\
\hline F45 & $1.251 \mathrm{E}-04$ & $2.353 \mathrm{E}-06$ & $1.127 \mathrm{E}-05$ & $4.729 \mathrm{E}-06$ & $8.972 E-02$ & $2.879 \mathrm{E}-06$ & $3.882 \mathrm{E}-06$ & $1.973 \mathrm{E}-05$ \\
\hline F46 & $1.734 \mathrm{E}-06$ & $4.320 \mathrm{E}-08$ & $1.734 \mathrm{E}-06$ & $1.734 \mathrm{E}-06$ & $1.734 \mathrm{E}-06$ & $1.734 \mathrm{E}-06$ & $1.734 \mathrm{E}-06$ & $1.734 \mathrm{E}-06$ \\
\hline F47 & $1.734 \mathrm{E}-06$ & $1.734 \mathrm{E}-06$ & $1.734 \mathrm{E}-06$ & $1.734 \mathrm{E}-06$ & $1.734 \mathrm{E}-06$ & $1.734 \mathrm{E}-06$ & $1.734 \mathrm{E}-06$ & $1.734 \mathrm{E}-06$ \\
\hline F48 & $1.734 \mathrm{E}-06$ & $1.734 \mathrm{E}-06$ & $1.734 \mathrm{E}-06$ & $2.563 \mathrm{E}-06$ & $1.734 \mathrm{E}-06$ & $1.734 \mathrm{E}-06$ & $1.734 \mathrm{E}-06$ & $1.734 \mathrm{E}-06$ \\
\hline F49 & $3.182 \mathrm{E}-06$ & $1.734 \mathrm{E}-06$ & $2.585 \mathrm{E}-03$ & $1.734 \mathrm{E}-06$ & $1.397 \mathrm{E}-02$ & $6.435 \mathrm{E}-01$ & $1.734 \mathrm{E}-06$ & $2.353 \mathrm{E}-06$ \\
\hline F50 & $1.734 \mathrm{E}-06$ & $1.734 \mathrm{E}-06$ & $1.734 \mathrm{E}-06$ & $1.734 \mathrm{E}-06$ & $1.734 \mathrm{E}-06$ & $1.734 \mathrm{E}-06$ & $1.734 \mathrm{E}-06$ & $1.734 \mathrm{E}-06$ \\
\hline F51 & $1.734 \mathrm{E}-06$ & $1.734 \mathrm{E}-06$ & $1.734 \mathrm{E}-06$ & $1.734 \mathrm{E}-06$ & $1.734 \mathrm{E}-06$ & $1.734 \mathrm{E}-06$ & $1.734 \mathrm{E}-06$ & $1.734 \mathrm{E}-06$ \\
\hline F52 & $1.734 \mathrm{E}-06$ & $1.734 \mathrm{E}-06$ & $1.734 \mathrm{E}-06$ & $1.734 \mathrm{E}-06$ & $1.734 \mathrm{E}-06$ & $1.734 \mathrm{E}-06$ & $1.734 \mathrm{E}-06$ & $1.722 \mathrm{E}-06$ \\
\hline F53 & $1.734 \mathrm{E}-06$ & $1.734 \mathrm{E}-06$ & $1.734 \mathrm{E}-06$ & $1.734 \mathrm{E}-06$ & $1.734 \mathrm{E}-06$ & $1.734 \mathrm{E}-06$ & $1.734 \mathrm{E}-06$ & $1.734 \mathrm{E}-06$ \\
\hline RANK & 1 & 2 & 3 & 11 & 4 & 9 & 12 & 8 \\
\hline
\end{tabular}

Table A4 (continued) $p$-value of Wilcoxon test obtained from comparison with traditional algorithms on 53 functions

\begin{tabular}{|c|c|c|c|}
\hline Function & WOA & GWO & GSA \\
\hline $\mathrm{F} 1$ & $1.000 E+00$ & $1.000 \mathrm{E}+00$ & $1.734 \mathrm{E}-06$ \\
\hline $\mathrm{F} 2$ & $1.000 \mathrm{E}+00$ & $1.000 \mathrm{E}+00$ & $1.734 \mathrm{E}-06$ \\
\hline F3 & $1.734 \mathrm{E}-06$ & $2.563 \mathrm{E}-06$ & $1.734 \mathrm{E}-06$ \\
\hline F4 & $1.734 \mathrm{E}-06$ & $1.734 \mathrm{E}-06$ & $1.734 \mathrm{E}-06$ \\
\hline F5 & $1.734 \mathrm{E}-06$ & $1.734 \mathrm{E}-06$ & $1.734 \mathrm{E}-06$ \\
\hline F6 & $1.734 \mathrm{E}-06$ & $1.734 \mathrm{E}-06$ & $1.734 \mathrm{E}-06$ \\
\hline F7 & $1.359 \mathrm{E}-04$ & $3.589 \mathrm{E}-04$ & $1.734 \mathrm{E}-06$ \\
\hline F8 & $1.734 \mathrm{E}-06$ & $1.734 \mathrm{E}-06$ & $1.734 \mathrm{E}-06$ \\
\hline F9 & $1.000 \mathrm{E}+00$ & $1.000 \mathrm{E}+00$ & $1.734 \mathrm{E}-06$ \\
\hline F10 & $6.104 \mathrm{E}-05$ & $2.572 \mathrm{E}-07$ & $1.734 \mathrm{E}-06$ \\
\hline F11 & $1.000 \mathrm{E}+00$ & $1.000 \mathrm{E}+00$ & $1.734 \mathrm{E}-06$ \\
\hline F12 & $1.734 \mathrm{E}-06$ & $1.734 \mathrm{E}-06$ & $1.734 \mathrm{E}-06$ \\
\hline F13 & $1.734 \mathrm{E}-06$ & $1.734 \mathrm{E}-06$ & $1.734 \mathrm{E}-06$ \\
\hline F14 & $3.196 \mathrm{E}-06$ & $1.734 \mathrm{E}-06$ & $1.734 \mathrm{E}-06$ \\
\hline F15 & $1.734 \mathrm{E}-06$ & $1.734 \mathrm{E}-06$ & $1.734 \mathrm{E}-06$ \\
\hline F16 & $2.441 \mathrm{E}-04$ & $1.734 \mathrm{E}-06$ & $1.734 \mathrm{E}-06$ \\
\hline F17 & $1.734 \mathrm{E}-06$ & $1.734 \mathrm{E}-06$ & $1.734 \mathrm{E}-06$ \\
\hline F18 & $1.734 \mathrm{E}-06$ & $1.734 \mathrm{E}-06$ & $1.734 \mathrm{E}-06$ \\
\hline F19 & $1.734 \mathrm{E}-06$ & $1.734 \mathrm{E}-06$ & $1.734 \mathrm{E}-06$ \\
\hline F20 & $1.734 \mathrm{E}-06$ & $1.734 \mathrm{E}-06$ & $1.734 \mathrm{E}-06$ \\
\hline F21 & $3.709 E-01$ & $3.709 \mathrm{E}-01$ & $7.514 \mathrm{E}-05$ \\
\hline
\end{tabular}




\begin{tabular}{|c|c|c|c|}
\hline $\mathrm{F} 22$ & $2.059 \mathrm{E}-01$ & $2.059 \mathrm{E}-01$ & $7.521 \mathrm{E}-02$ \\
\hline F23 & $2.059 \mathrm{E}-01$ & $2.059 \mathrm{E}-01$ & $1.566 \mathrm{E}-02$ \\
\hline $\mathrm{F} 24$ & $1.734 \mathrm{E}-06$ & $1.734 \mathrm{E}-06$ & $1.734 \mathrm{E}-06$ \\
\hline $\mathrm{F} 25$ & $1.734 \mathrm{E}-06$ & $1.734 \mathrm{E}-06$ & $1.734 \mathrm{E}-06$ \\
\hline F26 & $1.734 \mathrm{E}-06$ & $1.734 \mathrm{E}-06$ & $1.734 \mathrm{E}-06$ \\
\hline $\mathrm{F} 27$ & $1.734 \mathrm{E}-06$ & $1.734 \mathrm{E}-06$ & $4.779 \mathrm{E}-01$ \\
\hline F28 & $1.734 \mathrm{E}-06$ & $1.734 \mathrm{E}-06$ & $1.734 \mathrm{E}-06$ \\
\hline $\mathrm{F} 29$ & $1.734 \mathrm{E}-06$ & $1.799 \mathrm{E}-05$ & $1.734 \mathrm{E}-06$ \\
\hline $\mathrm{F} 30$ & $1.734 \mathrm{E}-06$ & $1.734 \mathrm{E}-06$ & $1.734 \mathrm{E}-06$ \\
\hline $\mathrm{F} 31$ & $1.734 \mathrm{E}-06$ & $1.734 \mathrm{E}-06$ & $1.734 \mathrm{E}-06$ \\
\hline $\mathrm{F} 32$ & $1.921 \mathrm{E}-06$ & $9.711 \mathrm{E}-05$ & $1.734 \mathrm{E}-06$ \\
\hline F33 & $1.734 \mathrm{E}-06$ & $1.734 \mathrm{E}-06$ & $1.734 \mathrm{E}-06$ \\
\hline F34 & $1.734 \mathrm{E}-06$ & $1.566 \mathrm{E}-02$ & $5.216 \mathrm{E}-06$ \\
\hline F35 & $1.734 \mathrm{E}-06$ & $3.112 \mathrm{E}-05$ & $1.734 \mathrm{E}-06$ \\
\hline F36 & $6.884 E-01$ & $5.752 \mathrm{E}-06$ & $1.734 \mathrm{E}-06$ \\
\hline F37 & $4.196 \mathrm{E}-04$ & $6.156 \mathrm{E}-04$ & $5.984 E-02$ \\
\hline F38 & $1.734 \mathrm{E}-06$ & $3.724 \mathrm{E}-05$ & $4.492 \mathrm{E}-02$ \\
\hline F39 & $1.734 \mathrm{E}-06$ & $2.052 \mathrm{E}-04$ & $1.734 \mathrm{E}-06$ \\
\hline F40 & $1.734 \mathrm{E}-06$ & $1.734 \mathrm{E}-06$ & $1.734 \mathrm{E}-06$ \\
\hline F41 & $7.712 \mathrm{E}-04$ & $2.831 \mathrm{E}-04$ & $1.734 \mathrm{E}-06$ \\
\hline F42 & $1.494 \mathrm{E}-05$ & $6.339 \mathrm{E}-06$ & $6.156 \mathrm{E}-04$ \\
\hline F43 & $1.734 \mathrm{E}-06$ & $1.734 \mathrm{E}-06$ & $1.734 \mathrm{E}-06$ \\
\hline $\mathrm{F} 44$ & $1.734 \mathrm{E}-06$ & $1.360 \mathrm{E}-05$ & $8.188 \mathrm{E}-05$ \\
\hline F45 & $1.477 \mathrm{E}-04$ & $5.320 \mathrm{E}-03$ & $1.734 \mathrm{E}-06$ \\
\hline F46 & $3.790 \mathrm{E}-06$ & $1.734 \mathrm{E}-06$ & $1.734 \mathrm{E}-06$ \\
\hline F47 & $1.734 \mathrm{E}-06$ & $1.734 \mathrm{E}-06$ & $1.734 \mathrm{E}-06$ \\
\hline F48 & $1.318 \mathrm{E}-04$ & $1.229 \mathrm{E}-05$ & $1.734 \mathrm{E}-06$ \\
\hline F49 & $2.849 \mathrm{E}-02$ & $8.972 E-02$ & $5.307 \mathrm{E}-05$ \\
\hline F50 & $1.734 \mathrm{E}-06$ & $1.734 \mathrm{E}-06$ & $1.734 \mathrm{E}-06$ \\
\hline F51 & $2.563 \mathrm{E}-06$ & $1.734 \mathrm{E}-06$ & $1.734 \mathrm{E}-06$ \\
\hline F52 & $1.720 \mathrm{E}-06$ & $1.734 \mathrm{E}-06$ & $1.734 \mathrm{E}-06$ \\
\hline F53 & $1.734 \mathrm{E}-06$ & $1.734 \mathrm{E}-06$ & $1.734 \mathrm{E}-06$ \\
\hline RANK & 6 & 5 & 7 \\
\hline
\end{tabular}

Table A5 $p$-value of Wilcoxon test obtained from comparison with advanced algorithms on 53 functions

\begin{tabular}{|c|c|c|c|c|c|c|c|c|c|}
\hline Fun & m_SCA & OBSCA & MWOA & IWOA & CDLOB & $\mathrm{CBA}$ & CGPSO & ALCPSO & DECLS \\
\hline $\mathrm{F} 1$ & $1.000 \mathrm{E}+00$ & $1.734 \mathrm{E}-06$ & $1.734 \mathrm{E}-06$ & $1.000 \mathrm{E}+00$ & $1.734 \mathrm{E}-06$ & $1.734 \mathrm{E}-06$ & $1.734 \mathrm{E}-06$ & $1.734 \mathrm{E}-06$ & $1.734 \mathrm{E}-06$ \\
\hline $\mathrm{F} 2$ & $1.000 \mathrm{E}+00$ & $1.734 \mathrm{E}-06$ & $1.734 \mathrm{E}-06$ & $1.000 \mathrm{E}+00$ & $1.734 \mathrm{E}-06$ & $1.734 \mathrm{E}-06$ & $1.734 \mathrm{E}-06$ & $1.734 \mathrm{E}-06$ & $1.734 \mathrm{E}-06$ \\
\hline F3 & $4.378 \mathrm{E}-04$ & $1.734 \mathrm{E}-06$ & $1.734 \mathrm{E}-06$ & $1.734 \mathrm{E}-06$ & $1.734 \mathrm{E}-06$ & $1.734 \mathrm{E}-06$ & $1.734 \mathrm{E}-06$ & $1.734 \mathrm{E}-06$ & $1.734 \mathrm{E}-06$ \\
\hline F4 & $1.734 \mathrm{E}-06$ & $1.734 \mathrm{E}-06$ & $2.931 \mathrm{E}-04$ & $1.734 \mathrm{E}-06$ & $1.734 \mathrm{E}-06$ & $1.734 \mathrm{E}-06$ & $1.734 \mathrm{E}-06$ & $1.734 \mathrm{E}-06$ & $1.734 \mathrm{E}-06$ \\
\hline F5 & $1.734 \mathrm{E}-06$ & $1.734 \mathrm{E}-06$ & $1.734 \mathrm{E}-06$ & $1.734 \mathrm{E}-06$ & $7.036 \mathrm{E}-01$ & $6.892 \mathrm{E}-05$ & $1.734 \mathrm{E}-06$ & $7.499 \mathrm{E}-01$ & $1.734 \mathrm{E}-06$ \\
\hline F6 & $1.734 \mathrm{E}-06$ & $1.734 \mathrm{E}-06$ & $1.734 \mathrm{E}-06$ & $1.734 \mathrm{E}-06$ & $1.734 \mathrm{E}-06$ & $1.734 \mathrm{E}-06$ & $1.734 \mathrm{E}-06$ & $1.734 \mathrm{E}-06$ & $1.734 \mathrm{E}-06$ \\
\hline F7 & $2.623 E-01$ & $1.734 \mathrm{E}-06$ & $1.734 \mathrm{E}-06$ & $4.070 \mathrm{E}-02$ & $1.734 \mathrm{E}-06$ & $1.734 \mathrm{E}-06$ & $5.287 \mathrm{E}-04$ & $1.734 \mathrm{E}-06$ & $6.836 \mathrm{E}-03$ \\
\hline F8 & $1.734 \mathrm{E}-06$ & $1.734 \mathrm{E}-06$ & $1.713 \mathrm{E}-06$ & $1.734 \mathrm{E}-06$ & $1.734 \mathrm{E}-06$ & $1.734 \mathrm{E}-06$ & $1.734 \mathrm{E}-06$ & $1.730 \mathrm{E}-06$ & $5.037 \mathrm{E}-04$ \\
\hline F9 & $1.000 \mathrm{E}+00$ & $1.000 \mathrm{E}+00$ & $1.734 \mathrm{E}-06$ & $1.000 \mathrm{E}+00$ & $1.734 \mathrm{E}-06$ & $1.734 \mathrm{E}-06$ & $1.733 \mathrm{E}-06$ & $1.734 \mathrm{E}-06$ & $2.500 \mathrm{E}-01$ \\
\hline F10 & $6.799 \mathrm{E}-08$ & $4.320 \mathrm{E}-08$ & $1.734 \mathrm{E}-06$ & $6.334 \mathrm{E}-05$ & $1.713 \mathrm{E}-06$ & $1.734 \mathrm{E}-06$ & $1.734 \mathrm{E}-06$ & $1.709 \mathrm{E}-06$ & $6.799 \mathrm{E}-08$ \\
\hline F11 & $1.000 \mathrm{E}+00$ & $1.000 \mathrm{E}+00$ & $1.734 \mathrm{E}-06$ & $1.000 \mathrm{E}+00$ & $1.734 \mathrm{E}-06$ & $5.606 \mathrm{E}-06$ & $1.734 \mathrm{E}-06$ & $2.696 \mathrm{E}-05$ & $1.000 E+00$ \\
\hline
\end{tabular}




\begin{tabular}{|c|c|c|c|c|c|c|c|c|c|}
\hline F12 & $1.734 \mathrm{E}-06$ & $1.734 \mathrm{E}-06$ & $1.734 \mathrm{E}-06$ & $1.734 \mathrm{E}-06$ & $1.734 \mathrm{E}-06$ & $1.734 \mathrm{E}-06$ & $1.734 \mathrm{E}-06$ & $7.813 \mathrm{E}-01$ & $1.734 \mathrm{E}-06$ \\
\hline F13 & $1.734 \mathrm{E}-06$ & $1.734 \mathrm{E}-06$ & $1.734 \mathrm{E}-06$ & $1.734 \mathrm{E}-06$ & $1.734 \mathrm{E}-06$ & $1.734 \mathrm{E}-06$ & $1.734 \mathrm{E}-06$ & $6.432 \mathrm{E}-01$ & $1.734 \mathrm{E}-06$ \\
\hline F14 & $1.734 \mathrm{E}-06$ & $1.734 \mathrm{E}-06$ & $1.734 \mathrm{E}-06$ & $5.388 \mathrm{E}-07$ & $1.734 \mathrm{E}-06$ & $8.277 \mathrm{E}-06$ & $1.645 \mathrm{E}-06$ & $1.000 \mathrm{E}+00$ & $5.000 \mathrm{E}-01$ \\
\hline F15 & $1.734 \mathrm{E}-06$ & $1.734 \mathrm{E}-06$ & $1.734 \mathrm{E}-06$ & $1.734 \mathrm{E}-06$ & $1.734 \mathrm{E}-06$ & $1.734 \mathrm{E}-06$ & $1.734 \mathrm{E}-06$ & $3.106 \mathrm{E}-05$ & $9.754 \mathrm{E}-01$ \\
\hline F16 & $1.734 \mathrm{E}-06$ & $1.734 \mathrm{E}-06$ & $1.734 \mathrm{E}-06$ & $7.813 \mathrm{E}-03$ & $1.734 \mathrm{E}-06$ & $1.734 \mathrm{E}-06$ & $1.734 \mathrm{E}-06$ & $1.000 \mathrm{E}+00$ & $1.000 \mathrm{E}+00$ \\
\hline F17 & $1.734 \mathrm{E}-06$ & $1.734 \mathrm{E}-06$ & $1.734 \mathrm{E}-06$ & $1.733 \mathrm{E}-06$ & $1.734 \mathrm{E}-06$ & $1.734 \mathrm{E}-06$ & $1.734 \mathrm{E}-06$ & $1.000 \mathrm{E}+00$ & $1.000 \mathrm{E}+00$ \\
\hline F18 & $1.734 \mathrm{E}-06$ & $1.734 \mathrm{E}-06$ & $1.734 \mathrm{E}-06$ & $1.733 \mathrm{E}-06$ & $1.734 \mathrm{E}-06$ & $1.734 \mathrm{E}-06$ & $1.734 \mathrm{E}-06$ & $5.699 \mathrm{E}-05$ & $1.000 \mathrm{E}+00$ \\
\hline F19 & $1.734 \mathrm{E}-06$ & $1.734 \mathrm{E}-06$ & $1.734 \mathrm{E}-06$ & $1.732 \mathrm{E}-06$ & $1.734 \mathrm{E}-06$ & $1.734 \mathrm{E}-06$ & $1.734 \mathrm{E}-06$ & $1.000 \mathrm{E}+00$ & $1.000 \mathrm{E}+00$ \\
\hline F20 & $1.734 \mathrm{E}-06$ & $3.112 \mathrm{E}-05$ & $1.734 \mathrm{E}-06$ & $1.734 \mathrm{E}-06$ & $1.734 \mathrm{E}-06$ & $5.216 \mathrm{E}-06$ & $1.734 \mathrm{E}-06$ & $7.813 \mathrm{E}-03$ & $1.000 \mathrm{E}+00$ \\
\hline F21 & $4.716 \mathrm{E}-02$ & $4.716 \mathrm{E}-02$ & $1.734 \mathrm{E}-06$ & $4.716 \mathrm{E}-02$ & $2.703 \mathrm{E}-02$ & $1.833 \mathrm{E}-03$ & $4.716 \mathrm{E}-02$ & $1.600 \mathrm{E}-01$ & $1.221 \mathrm{E}-04$ \\
\hline F22 & $1.109 \mathrm{E}-01$ & $1.020 \mathrm{E}-01$ & $1.734 \mathrm{E}-06$ & $1.020 \mathrm{E}-01$ & $9.590 \mathrm{E}-01$ & $2.802 E-01$ & $1.020 \mathrm{E}-01$ & $4.272 \mathrm{E}-04$ & $2.441 \mathrm{E}-04$ \\
\hline F23 & $6.733 E-01$ & $6.435 E-01$ & $3.182 \mathrm{E}-06$ & $6.435 E-01$ & $7.521 E-02$ & $6.035 \mathrm{E}-03$ & $6.435 E-01$ & $1.953 \mathrm{E}-03$ & $9.766 \mathrm{E}-04$ \\
\hline F24 & $1.734 \mathrm{E}-06$ & $1.734 \mathrm{E}-06$ & $1.734 \mathrm{E}-06$ & $1.734 \mathrm{E}-06$ & $5.999 \mathrm{E}-01$ & $1.734 \mathrm{E}-06$ & $1.734 \mathrm{E}-06$ & $1.734 \mathrm{E}-06$ & $1.734 \mathrm{E}-06$ \\
\hline F25 & $1.734 \mathrm{E}-06$ & $1.734 \mathrm{E}-06$ & $1.734 \mathrm{E}-06$ & $1.734 \mathrm{E}-06$ & $1.566 \mathrm{E}-02$ & $6.268 \mathrm{E}-02$ & $1.734 \mathrm{E}-06$ & $9.316 \mathrm{E}-06$ & $6.339 \mathrm{E}-06$ \\
\hline F26 & $1.734 \mathrm{E}-06$ & $1.734 \mathrm{E}-06$ & $1.734 \mathrm{E}-06$ & $1.734 \mathrm{E}-06$ & $1.734 \mathrm{E}-06$ & $5.216 \mathrm{E}-06$ & $1.734 \mathrm{E}-06$ & $9.368 \mathrm{E}-02$ & $1.245 \mathrm{E}-02$ \\
\hline F27 & $1.734 \mathrm{E}-06$ & $1.734 \mathrm{E}-06$ & $1.734 \mathrm{E}-06$ & $2.879 \mathrm{E}-06$ & $9.842 \mathrm{E}-03$ & $2.843 \mathrm{E}-05$ & $1.204 \mathrm{E}-01$ & $4.729 \mathrm{E}-06$ & $6.892 \mathrm{E}-05$ \\
\hline F28 & $1.734 \mathrm{E}-06$ & $1.734 \mathrm{E}-06$ & $1.734 \mathrm{E}-06$ & $1.734 \mathrm{E}-06$ & $1.734 \mathrm{E}-06$ & $2.353 \mathrm{E}-06$ & $1.734 \mathrm{E}-06$ & $1.734 \mathrm{E}-06$ & $1.734 \mathrm{E}-06$ \\
\hline F29 & $1.494 \mathrm{E}-05$ & $1.734 \mathrm{E}-06$ & $1.734 \mathrm{E}-06$ & $1.734 \mathrm{E}-06$ & $1.734 \mathrm{E}-06$ & $1.734 \mathrm{E}-06$ & $1.734 \mathrm{E}-06$ & $6.143 E-01$ & $2.879 \mathrm{E}-06$ \\
\hline F30 & $1.734 \mathrm{E}-06$ & $1.734 \mathrm{E}-06$ & $1.734 \mathrm{E}-06$ & $1.734 \mathrm{E}-06$ & $5.287 \mathrm{E}-04$ & $2.957 \mathrm{E}-03$ & $1.734 \mathrm{E}-06$ & $2.255 \mathrm{E}-03$ & $1.712 \mathrm{E}-06$ \\
\hline F31F & $1.734 \mathrm{E}-06$ & $1.734 \mathrm{E}-06$ & $1.734 \mathrm{E}-06$ & $1.734 \mathrm{E}-06$ & $1.734 \mathrm{E}-06$ & $1.734 \mathrm{E}-06$ & $1.734 \mathrm{E}-06$ & $1.734 \mathrm{E}-06$ & $9.208 \mathrm{E}-05$ \\
\hline F32 & $6.339 \mathrm{E}-06$ & $1.734 \mathrm{E}-06$ & $1.734 \mathrm{E}-06$ & $1.734 \mathrm{E}-06$ & $1.734 \mathrm{E}-06$ & $1.734 \mathrm{E}-06$ & $1.734 \mathrm{E}-06$ & $5.667 \mathrm{E}-03$ & $5.304 \mathrm{E}-01$ \\
\hline F33 & $1.734 \mathrm{E}-06$ & $1.734 \mathrm{E}-06$ & $1.734 \mathrm{E}-06$ & $1.734 \mathrm{E}-06$ & $1.734 \mathrm{E}-06$ & $1.734 \mathrm{E}-06$ & $1.734 \mathrm{E}-06$ & $1.734 \mathrm{E}-06$ & $1.734 \mathrm{E}-06$ \\
\hline F34 & $3.405 \mathrm{E}-05$ & $1.734 \mathrm{E}-06$ & $1.734 \mathrm{E}-06$ & $1.921 \mathrm{E}-06$ & $1.734 \mathrm{E}-06$ & $1.734 \mathrm{E}-06$ & $1.734 \mathrm{E}-06$ & $6.639 \mathrm{E}-04$ & $1.734 \mathrm{E}-06$ \\
\hline F35 & $1.734 \mathrm{E}-06$ & $1.734 \mathrm{E}-06$ & $1.734 \mathrm{E}-06$ & $1.734 \mathrm{E}-06$ & $3.589 \mathrm{E}-04$ & $1.734 \mathrm{E}-06$ & $1.734 \mathrm{E}-06$ & $2.353 \mathrm{E}-06$ & $1.734 \mathrm{E}-06$ \\
\hline F36 & $2.765 \mathrm{E}-03$ & $1.734 \mathrm{E}-06$ & $1.734 \mathrm{E}-06$ & $6.583 E-01$ & $1.064 \mathrm{E}-01$ & $1.470 \mathrm{E}-01$ & $6.639 \mathrm{E}-04$ & $7.655 \mathrm{E}-01$ & $1.734 \mathrm{E}-06$ \\
\hline F37 & $2.603 \mathrm{E}-06$ & $1.734 \mathrm{E}-06$ & $1.734 \mathrm{E}-06$ & $9.627 \mathrm{E}-04$ & $4.492 \mathrm{E}-02$ & $1.589 \mathrm{E}-01$ & $4.390 \mathrm{E}-03$ & $2.304 \mathrm{E}-02$ & $2.957 \mathrm{E}-03$ \\
\hline F38 & $1.734 \mathrm{E}-06$ & $1.734 \mathrm{E}-06$ & $1.734 \mathrm{E}-06$ & $1.734 \mathrm{E}-06$ & $1.734 \mathrm{E}-06$ & $1.921 \mathrm{E}-06$ & $1.359 \mathrm{E}-04$ & $4.165 \mathrm{E}-01$ & $4.405 \mathrm{E}-01$ \\
\hline F39 & $1.127 \mathrm{E}-05$ & $1.734 \mathrm{E}-06$ & $1.734 \mathrm{E}-06$ & $1.734 \mathrm{E}-06$ & $1.734 \mathrm{E}-06$ & $1.734 \mathrm{E}-06$ & $1.734 \mathrm{E}-06$ & $2.353 \mathrm{E}-06$ & $1.734 \mathrm{E}-06$ \\
\hline F40 & $1.734 \mathrm{E}-06$ & $1.734 \mathrm{E}-06$ & $1.734 \mathrm{E}-06$ & $1.734 \mathrm{E}-06$ & $8.188 \mathrm{E}-05$ & $2.353 \mathrm{E}-06$ & $1.921 \mathrm{E}-06$ & $7.691 \mathrm{E}-06$ & $1.734 \mathrm{E}-06$ \\
\hline F41 & $1.734 \mathrm{E}-06$ & $1.734 \mathrm{E}-06$ & $1.734 \mathrm{E}-06$ & $1.254 \mathrm{E}-01$ & $3.327 \mathrm{E}-02$ & $2.067 \mathrm{E}-02$ & $1.734 \mathrm{E}-06$ & $1.957 \mathrm{E}-02$ & $9.711 \mathrm{E}-05$ \\
\hline F42 & $1.734 \mathrm{E}-06$ & $1.734 \mathrm{E}-06$ & $1.734 \mathrm{E}-06$ & $1.734 \mathrm{E}-06$ & $1.734 \mathrm{E}-06$ & $1.734 \mathrm{E}-06$ & $1.734 \mathrm{E}-06$ & $1.114 \mathrm{E}-03$ & $1.846 \mathrm{E}-01$ \\
\hline F43 & $1.734 \mathrm{E}-06$ & $1.734 \mathrm{E}-06$ & $1.734 \mathrm{E}-06$ & $1.734 \mathrm{E}-06$ & $1.734 \mathrm{E}-06$ & $1.734 \mathrm{E}-06$ & $1.734 \mathrm{E}-06$ & $1.734 \mathrm{E}-06$ & $1.734 \mathrm{E}-06$ \\
\hline F44 & $3.182 \mathrm{E}-06$ & $1.734 \mathrm{E}-06$ & $1.734 \mathrm{E}-06$ & $1.734 \mathrm{E}-06$ & $7.271 \mathrm{E}-03$ & $3.160 \mathrm{E}-02$ & $1.799 \mathrm{E}-05$ & $8.774 E-01$ & $1.734 \mathrm{E}-06$ \\
\hline F45 & $9.271 \mathrm{E}-03$ & $2.879 \mathrm{E}-06$ & $1.734 \mathrm{E}-06$ & $7.731 \mathrm{E}-03$ & $2.353 \mathrm{E}-06$ & $3.182 \mathrm{E}-06$ & $1.397 \mathrm{E}-02$ & $3.820 \mathrm{E}-01$ & $3.182 \mathrm{E}-06$ \\
\hline F46 & $1.734 \mathrm{E}-06$ & $1.734 \mathrm{E}-06$ & $1.734 \mathrm{E}-06$ & $1.734 \mathrm{E}-06$ & $1.734 \mathrm{E}-06$ & $1.734 \mathrm{E}-06$ & $1.734 \mathrm{E}-06$ & $1.734 \mathrm{E}-06$ & $1.734 \mathrm{E}-06$ \\
\hline F47 & $1.734 \mathrm{E}-06$ & $1.734 \mathrm{E}-06$ & $1.734 \mathrm{E}-06$ & $1.734 \mathrm{E}-06$ & $1.734 \mathrm{E}-06$ & $1.734 \mathrm{E}-06$ & $1.734 \mathrm{E}-06$ & $1.734 \mathrm{E}-06$ & $1.734 \mathrm{E}-06$ \\
\hline F48 & $1.734 \mathrm{E}-06$ & $1.250 \mathrm{E}-01$ & $1.734 \mathrm{E}-06$ & $1.964 \mathrm{E}-04$ & $1.734 \mathrm{E}-06$ & $1.734 \mathrm{E}-06$ & $1.734 \mathrm{E}-06$ & $1.734 \mathrm{E}-06$ & $1.734 \mathrm{E}-06$ \\
\hline F49 & $4.449 \mathrm{E}-05$ & $1.734 \mathrm{E}-06$ & $1.734 \mathrm{E}-06$ & $2.712 E-01$ & $7.813 E-01$ & $5.999 \mathrm{E}-01$ & $1.734 \mathrm{E}-06$ & $6.035 \mathrm{E}-03$ & $1.734 \mathrm{E}-06$ \\
\hline F50 & $1.734 \mathrm{E}-06$ & $1.734 \mathrm{E}-06$ & $1.734 \mathrm{E}-06$ & $1.734 \mathrm{E}-06$ & $1.734 \mathrm{E}-06$ & $1.734 \mathrm{E}-06$ & $1.734 \mathrm{E}-06$ & $1.734 \mathrm{E}-06$ & $1.734 \mathrm{E}-06$ \\
\hline F51 & $1.734 \mathrm{E}-06$ & $1.734 \mathrm{E}-06$ & $1.734 \mathrm{E}-06$ & $1.734 \mathrm{E}-06$ & $1.734 \mathrm{E}-06$ & $1.734 \mathrm{E}-06$ & $1.734 \mathrm{E}-06$ & $1.734 \mathrm{E}-06$ & $1.734 \mathrm{E}-06$ \\
\hline F52 & $1.734 \mathrm{E}-06$ & $1.734 \mathrm{E}-06$ & $1.734 \mathrm{E}-06$ & $1.663 \mathrm{E}-06$ & $1.734 \mathrm{E}-06$ & $1.734 \mathrm{E}-06$ & $1.734 \mathrm{E}-06$ & $1.734 \mathrm{E}-06$ & $1.734 \mathrm{E}-06$ \\
\hline F53 & $1.734 \mathrm{E}-06$ & $1.734 \mathrm{E}-06$ & $1.734 \mathrm{E}-06$ & $1.734 \mathrm{E}-06$ & $1.734 \mathrm{E}-06$ & $1.734 \mathrm{E}-06$ & $1.734 \mathrm{E}-06$ & $1.734 \mathrm{E}-06$ & $1.734 \mathrm{E}-06$ \\
\hline
\end{tabular}

